\title{
RESEARCH TOPICS in EMPIRICAL PROCESSES
}

by

\section{Christopher Ball}

Supervisor : Estate Khmaladze

A thesis

submitted to Victoria University of Wellington in fulfilment of the requirements for the degree of Master of Science

in Stochastic Processes in Finance and Insurance.

Victoria University of Wellington

2013 


\begin{abstract}
The first chapter consists of an overview of the theory of empirical processes, covering an introduction to empirical processes in $\mathbb{R}$, uniform empirical processes and function parametric empirical processes in SeCtion 1.1. SECtion 1.2 contains an overview of the theory related to the law of the iterated logarithm for Brownian motion and the modulus of continuity for Brownian motion. SECTION 1.3 contains the theory of the limiting processes for the empirical process, most importantly Brownian motion, Brownian bridge and the connections and relationships between them, with distributions of selected statistics of Brownian motion and Brownian bridge derived from reflection principles. SECTION 1.4 contains an overview of the theory required to prove central limit results for the empirical processes, covering the theory of the space $C$ and Donsker's theorem.
\end{abstract}

The second chapter covers research topics, starting with Fourier analysis of mixture distributions and associated theory in Section 2.1. Section 2.2 covers findings in a research problem about non-linear autoregressive processes. SECTION 2.3 introduces a martingale approach to testing a regression model. SECTION 2.4 links the theory of ranks and sequential ranks to the theory of empirical processes. 


\section{Contents}

1 General Theory of Empirical Processes 1

1.1 The empirical distribution function $\quad 1$

1.1.1 Glivenko-Cantelli theorem 1

1.1.2 Uniform empirical processes 8

1.1.3 Empirical processes indexed by functions 10

1.1.4 Multi-dimensional empirical process results 13

1.2 Law of the iterated logarithm and modulus of continuity

1.2.1 Law of the iterated logarithm 21

1.2.2 Levy modulus of continuity 30

1.3 Some limiting processes 33

\subsubsection{Gaussian processes 34 \\ 1.3.2 Reflection principles $\quad 49$}

1.4 Central limit theorem for empirical processes $\quad 59$

1.4.1 Theory of weak convergence $\quad 59$

1.4.2 Convergence in distribution 62

1.4.3 Prohorov's theorem 65 
1.4.4 The space $C \quad 68$

1.4.5 Construction of the Weiner measure 73

1.4.6 Central limit theorem for empirical processes $\quad 77$

2 Collection of Research Problems

2.1 Fourier Analysis of Mixtures

85

2.1.1 Characteristic Functions

85

2.1.2 Mixtures 91

2.2 Research Problem 99

2.3 New approach to testing regression 103

2.4 Ranks 110

2.4.1 Introduction to ranks 110

2.4.2 Applications 114

2.4.3 Sequential ranks 119

BIBLIOGRAPHY $\quad 127$ 


\section{List OF Figures}

Figure 1.1 Lema 1.2.4 23

Figure 1.2 Simulation of Brownian motion path 36

Figure 1.3 Simulation of Brownian Bridge path 36

Figure 1.4 TheOrem 1.3.29, Figure $1 \quad 52$

Figure 1.5 Theorem 1.3.29, Figure $2 \quad 53$

Figure $1.6 \quad$ Theorem 1.3.30, Figure $1 \quad 54$

Figure 1.7 Theorem 1.3.30, Figure 254

Figure 1.8 TheOrem $1.3 .32 \quad 57$

Figure 2.1 Mixture of normal densities with $\mu_{1}=-1.5, \mu_{2}=$ $0, \mu_{3}=1.5$ and $\sigma_{i}^{2}=1 . \quad 94$

Figure 2.2 Mixture of normal distributions with $\mu_{1}=-1.5, \mu_{2}=$ $0, \mu_{3}=1.5$ and $\sigma_{i}^{2}=1 . \quad 95$

FiguRE 2.3 Mixture of normal densities with $\mu_{1}=-2, \mu_{2}=-1$, $\mu_{3}=1, \mu_{4}=2$ and $\sigma_{i}^{2}=1 . \quad 96$

Figure 2.4 Mixture of normal distributions with $\mu_{1}=-2, \mu_{2}=$ $-1, \mu_{3}=1, \mu_{4}=2$ and $\sigma_{i}^{2}=1 . \quad 97$ 
Figure 2.5 Mixture of Cauchy densities with scale parameter $=1$ for all components and shift paramters $\mu=$ $(-1,0,1) \quad 98$

Figure 2.6 Section 2.2, Figure 1100

Figure 2.7 Section 2.2, Figure 2101

Figure 2.8 Section 2.2, Figure 3102 


\section{General Theory of Empirical Processes}

\subsection{THE EMPIRICAL DISTRIBUTION FUNCTION}

The first section outlines properties of the empirical distribution function, which is of fundamental importance to the study of empirical processes. We outline some of the theory relating to the empirical distribution function, such as the Glivenko-Cantelli theorem, and some extensions to the uniform empirical process and empirical processes indexed by function. The first section concludes with some inequalities related to the uniform empirical process.

\subsubsection{GLIVENKO-CANTELLI THEOREM}

Definition 1.1.1: The empirical distribution function $F_{n}(t)$ for a sample of size $n$ is defined as

$$
F_{n}(t)=\frac{1}{n} \sum_{i=1}^{n} \mathbb{I}_{\left\{X_{i} \leq t\right\}}=\frac{1}{n} \#\left\{1 \leq i \leq n: X_{i} \leq t\right\}
$$

It can be seen that $F_{n}(t)$ depends on both $t$ and $\omega(\in \Omega)$. For fixed $\omega, F_{n}(t)$ is a distribution function with a discrete measure assigning mass $\frac{1}{n}$ to each $X_{i}(\omega)$. For fixed $t \in \mathbb{R}, F_{n}(t)$ is a random variable taking values in $[0,1]$.

Lemma 1.1.2: The random variable $n F_{n}(t)$ has a binomial distribution with parameters $(n, F(t))$

Proof: Consider an individual $X_{i}$.

$$
\mathbb{P}\left\{\mathbb{I}_{\left\{X_{i} \leq t\right\}}=1\right\}=\mathbb{P}\left\{X_{i} \leq t\right\}=F(t)
$$


Therefore $n F_{n}(t)$ is the sum of $n$ independent and identically distributed Bernoulli random variables $\mathbb{I}_{\left\{X_{i} \leq t\right\}}$, therefore it is binomial with parameters $(n, F(t))$.

Corollary 1.1.3:

$$
E\left[F_{n}(t)\right]=F(t) \quad \text { and } \quad \operatorname{Var}\left(F_{n}(t)\right)=\frac{1}{n} F(t)(1-F(t))
$$

Proof: This follow directly from the empirical distribution function having a binomial distribution.

This implies that $F_{n}(t)$ is an unbiased estimator for $F(t)$. From the strong law of large numbers it follows

$$
F_{n}(t) \rightarrow F(t) \quad \text { almost surely }(n \rightarrow \infty) \text {. }
$$

The next question is if this convergence can be made uniform in $t$, which is answered in the next theorem.

Theorem 1.1.4: (Glivenko-Cantelli) For any distribution function $F(t)$

$$
\sup _{t \in \mathbb{R}}\left|F_{n}(t)-F(t)\right| \rightarrow 0 \quad \text { almost surely as } n \rightarrow \infty
$$

We prove the above theorem in 2 different ways. The first proof is based on the strong law of large numbers; the second proof is a modern proof which can be extended in various ways. The original papers are Glivenko (1933) and Cantelli (1933).

Proof: We need to show that $\forall m \in \mathbb{N}$

$$
\limsup _{n \rightarrow \infty}\left|F_{n}(t)-F(t)\right| \leq \frac{1}{m+1} \quad \text { almost surely }
$$


Choose $-\infty=t_{0, m}<t_{1, m} \leq \cdots \leq t_{m, m}<t_{m+1, m}=\infty$ so that

$$
F\left(t_{k, m^{-}}\right) \leq \frac{k}{m+1} \leq F\left(t_{k, m}\right) \quad \forall k \in\{1, \ldots, m\}
$$

As

$$
\left|F_{n}(t)-F(t)\right|=\max \left\{F_{n}(t)-F(t), F(t)-F_{n}(t)\right\}
$$

for any $t \in\left[t_{k-1, m}, t_{k, m}\right)$ and any $k \in\{1, \ldots, m+1\}$ we have

$$
\begin{aligned}
F_{n}(t)-F(t) & \leq F_{n}\left(t_{k, m^{-}}\right)-F\left(t_{k-1, m}\right) \\
& =F_{n}\left(t_{k, m^{-}}\right)-F\left(t_{k, m^{-}}\right)+F\left(t_{k, m^{-}}\right)-F\left(t_{k-1, m}\right) \\
& \leq\left|F_{n}\left(t_{k, m^{-}}\right)-F\left(t_{k, m^{-}}\right)\right|+\frac{1}{m+1}
\end{aligned}
$$

Similarly

$$
F(t)-F_{n}(t) \leq\left|F_{n}\left(t_{k-1, m^{-}}\right)-F\left(t_{k-1, m^{-}}\right)\right|+\frac{1}{m+1}
$$

Because $F_{n}(-\infty)-F(-\infty)=F_{n}(\infty)-F(\infty)=0$

$$
\begin{aligned}
\sup _{t \in \mathbb{R}}\left|F_{n}(t)-F(t)\right| \leq & \max _{1 \leq k \leq m}\left|F_{n}\left(t_{k, m^{-}}\right)-F\left(t_{k, m^{-}}\right)\right| \\
& +\max _{1 \leq k \leq m}\left|F_{n}\left(t_{k, m}\right)-F\left(t_{k, m}\right)\right|+\frac{1}{m+1}
\end{aligned}
$$

Using the strong law of large numbers, as $n \rightarrow \infty$,

$$
\begin{gathered}
F_{n}\left(t_{k, m^{-}}\right) \rightarrow F\left(t_{k, m^{-}}\right) \text {and } F_{n}\left(t_{k, m}\right) \rightarrow F\left(t_{k, m}\right) \quad \text { almost surely } \\
\Rightarrow \sup _{t \in \mathbb{R}}\left|F_{n}(t)-F(t)\right| \leq \frac{1}{m+1}
\end{gathered}
$$

Since $m$ was arbitrary, letting $m \rightarrow \infty$

$$
\sup _{t \in \mathbb{R}}\left|F_{n}(t)-F(t)\right| \rightarrow 0 \quad \text { almost surely }
$$

as $n \rightarrow \infty$. 
For the second proof, we first need the Hoeffding inequality for Rademacher variables. Hoeffding's inequality holds for more general random variables, see Hoeffing (1963). The next lemma is from Kosorok (2008).

Lemma 1.1.5: Let $X_{i}$ be Rademacher variables for $i=1,2, \ldots, n$, that is, $\mathbb{P}\{X=1\}=\mathbb{P}\{X=-1\}=\frac{1}{2}$. Then for fixed $a=\left(a_{1}, a_{2}, \ldots, a_{n}\right) \in \mathbb{R}^{n}$

$$
\mathbb{P}\left\{\left|\sum_{i=1}^{n} a_{i} X_{i}\right|>\lambda\right\} \leq 2 \exp \left(-\frac{\lambda^{2}}{2\|a\|^{2}}\right)
$$

where $\|a\|$ is the Euclidean norm.

Proof: For all $\lambda \in \mathbb{R}$

$$
\begin{aligned}
E\left[\exp \left(\lambda X_{i}\right)\right] & =\frac{\exp (\lambda)+\exp (-\lambda)}{2} \\
& =\sum_{i=0}^{\infty}\left(\frac{\lambda^{2 i}}{(2 i) !}\right) \leq \sum_{i=0}^{\infty}\left(\frac{\lambda^{2 i}}{2^{i}(i) !}\right)=\exp \left(\frac{\lambda^{2}}{2}\right)
\end{aligned}
$$

Therefore

$$
\mathbb{P}\left\{\sum_{i=1}^{n} a_{i} X_{i}>\lambda\right\}=\mathbb{P}\left\{\exp \left(s \sum_{i=1}^{n} a_{i} X_{i}\right)>\exp (\lambda s)\right\}
$$

By Markov's inequality

$$
\begin{aligned}
& \leq \exp (-\lambda s) E\left[\exp \left(s \sum_{i=1}^{n} a_{i} X_{i}\right)\right] \\
& \leq \exp (-\lambda s) \exp \left(\frac{s^{2}}{2}\|a\|^{2}\right)
\end{aligned}
$$

Choosing $s=\frac{\lambda}{\|a\|^{2}}$ gives

$$
\mathbb{P}\left\{\sum_{i=1}^{n} a_{i} X_{i}>\lambda\right\} \leq \exp \left(-\frac{\lambda^{2}}{2\|a\|^{2}}\right)
$$




$$
\begin{array}{r}
\text { As } \mathbb{P}\left\{\sum_{i=1}^{n} a_{i} X_{i}>\lambda\right\}=\mathbb{P}\left\{-\sum_{i=1}^{n} a_{i} X_{i}>\lambda\right\} \\
\mathbb{P}\left\{\left|\sum_{i=1}^{n} a_{i} X_{i}\right|>\lambda\right\} \leq 2 \exp \left(-\frac{\lambda^{2}}{2\|a\|^{2}}\right)
\end{array}
$$

Theorem 1.1.6: (Glivenko-Cantelli). For any distribution function $F(t)$

$$
\sup _{t \in \mathbb{R}}\left|F_{n}(t)-F(t)\right| \rightarrow 0 \quad \text { almost surely as } n \rightarrow \infty
$$

Proof:(Second proof)

Let $X_{1}, \ldots, X_{n}$ and $X_{1}^{\prime}, \ldots, X_{n}^{\prime}$ be 2 independent samples from distribution $F$. Then for any $\epsilon>0$ and $n>\frac{2}{\epsilon^{2}}$, using Chebyshev's inequality

$$
\begin{aligned}
\mathbb{P}\left\{\left|F_{n}^{\prime}(t)-F(t)\right| \leq \frac{\epsilon}{2}\right\} & \geq 1-\frac{E\left[\left|F_{n}^{\prime}(t)-F(t)\right|^{2}\right]}{\epsilon^{2}} \\
& =1-\frac{\operatorname{Var}\left(\left|F_{n}^{\prime}(t)-F(t)\right|\right)}{\epsilon^{2}} \\
& =1-\frac{\frac{1}{n} F(t)(1-F(t))}{\epsilon^{2}} \\
& \geq 1-\frac{1}{4 n \epsilon^{2}} \\
\Rightarrow \mathbb{P}\left\{\left|F_{n}^{\prime}(t)-F(t)\right| \leq \frac{\epsilon}{2}\right\} & \geq 1-\frac{1}{2}=\frac{1}{2}
\end{aligned}
$$

where $F_{n}$ and $F_{n}^{\prime}$ are the respective empirical distribution functions. Now select a random variable $\tau$, independent of $F_{n}^{\prime}$, so that $\left|F_{n}(\tau)-F(\tau)\right|>\epsilon$ on the event $\left\{\sup _{t \in \mathbb{R}}\left|F_{n}(t)-F(t)\right|>\epsilon\right\}$. Note that EQUATION 1.1 remains true 
when $t$ is replaced by $\tau$, even when we condition on $F_{n}$.

$$
\begin{aligned}
& \frac{1}{2} \mathbb{P}\left\{\sup _{t \in \mathbb{R}}\left|F_{n}(t)-F(t)\right|>\epsilon\right\} \\
& \leq \mathbb{P}\left\{\left|F_{n}^{\prime}(\tau)-F(\tau)\right| \leq \frac{\epsilon}{2}\left|\sup _{t \in \mathbb{R}}\right| F_{n}(t)-F(t) \mid>\epsilon\right\} \mathbb{P}\left\{\sup _{t \in \mathbb{R}}\left|F_{n}(t)-F(t)\right|>\epsilon\right\} \\
& =\mathbb{P}\left\{\left|F_{n}^{\prime}(\tau)-F(\tau)\right| \leq \frac{\epsilon}{2}, \sup _{t \in \mathbb{R}}\left|F_{n}(t)-F(t)\right|>\epsilon\right\}
\end{aligned}
$$

Using the definition of $\tau$

$$
\begin{aligned}
& =\mathbb{P}\left\{\left|F_{n}^{\prime}(\tau)-F(\tau)\right| \leq \frac{\epsilon}{2}, \quad\left|F_{n}(\tau)-F(\tau)\right|>\epsilon\right\} \\
& \leq \mathbb{P}\left\{\left|F_{n}(\tau)-F_{n}^{\prime}(\tau)\right|>\frac{\epsilon}{2}\right\} \\
& \leq \mathbb{P}\left\{\sup _{t \in \mathbb{R}}\left|F_{n}(t)-F_{n}^{\prime}(t)\right|>\frac{\epsilon}{2}\right\}
\end{aligned}
$$

The next step is to define $\eta_{i}, i \in\{1, \ldots, n\}$ as independent and identically distributed Rademacher variables,

$$
\mathbb{P}\left\{\eta_{i}=-1\right\}=\mathbb{P}\left\{\eta_{i}=1\right\}=\frac{1}{2}
$$

where $\eta_{i}$ is independent of $X_{i}$ and $X_{i}^{\prime}$. Observe that

$$
\begin{gathered}
\mathcal{L}\left(\mathbb{I}_{\left\{X_{i} \leq t\right\}}-\mathbb{I}_{\left\{X_{i}^{\prime} \leq t\right\}} ; i=1, \ldots, n ; t \in \mathbb{R}\right) \\
=\mathcal{L}\left(\eta_{i}\left(\mathbb{I}_{\left\{X_{i} \leq t\right\}}-\mathbb{I}_{\left\{X_{i}^{\prime} \leq t\right\}}\right) ; i=1, \ldots, n ; t \in \mathbb{R}\right)
\end{gathered}
$$

where $\mathcal{L}()$ is the law. Hence

$$
\begin{aligned}
& \mathbb{P}\left\{\sup _{t \in \mathbb{R}}\left|F_{n}(t)-F_{n}^{\prime}(t)\right|>\frac{\epsilon}{2}\right\} \\
& =\mathbb{P}\left\{\sup _{t \in \mathbb{R}}\left|\frac{1}{n} \sum_{i=1}^{n}\left(\eta_{i}\left(\mathbb{I}_{\left\{X_{i} \leq t\right\}}-\mathbb{I}_{\left\{X_{i}^{\prime} \leq t\right\}}\right)\right)\right|>\frac{\epsilon}{2}\right\} \\
& \leq \mathbb{P}\left\{\sup _{t \in \mathbb{R}}\left|\frac{1}{n} \sum_{i=1}^{n} \eta_{i} \mathbb{I}_{\left\{X_{i} \leq t\right\}}\right|>\frac{\epsilon}{4}\right\}+\mathbb{P}\left\{\sup _{t \in \mathbb{R}}\left|\frac{1}{n} \sum_{i=1}^{n} \eta_{i} \mathbb{I}_{\left\{X_{i}^{\prime} \leq t\right\}}\right|>\frac{\epsilon}{4}\right\}
\end{aligned}
$$




$$
=2 \mathbb{P}\left\{\sup _{t \in \mathbb{R}}\left|F_{n}^{s}(t)\right|>\frac{\epsilon}{4}\right\}
$$

where $F_{n}^{s}(t)=\frac{1}{n} \sum_{i=1}^{n} \eta_{i} \mathbb{I}_{\left\{X_{i}^{\prime} \leq t\right\}}, t \in \mathbb{R}$. Using EQUATION 1.2 and EQUATION 1.3 we get

$$
\mathbb{P}\left\{\sup _{t \in \mathbb{R}}\left|F_{n}(t)-F(t)\right|>\epsilon\right\} \leq 4 \mathbb{P}\left\{\sup _{t \in \mathbb{R}}\left|F_{n}^{s}(t)\right|>\frac{\epsilon}{4}\right\} .
$$

We now want to derive bounds for EQUATION 1.4. Conditioning on the values of $X_{i}$ we see that

$$
\begin{aligned}
& \mathbb{P}\left\{\sup _{t \in \mathbb{R}}\left|F_{n}^{s}(t)\right|>\frac{\epsilon}{4} \mid X_{i}=x_{i} ; i \in\{1, \ldots, n\}\right\} \\
& \leq \sum_{i=1}^{n} \mathbb{P}\left\{\left|F_{n}^{s}(t)\right|>\frac{\epsilon}{4} \mid X_{i}=x_{i} ; i \in\{1, \ldots, n\}\right\}
\end{aligned}
$$

Using LEMMA 1.1.5, with $\lambda=\frac{n \epsilon}{4}$ and $a=\left(\mathbb{I}_{\left\{X_{1} \leq t\right\}}, \ldots, \mathbb{I}_{\left\{X_{n} \leq t\right\}}\right)$

$$
\begin{aligned}
\mathbb{P}\left\{\left|F_{n}^{s}(t)\right|>\frac{\epsilon}{4} \mid X_{i}=x_{i} ; i \in\{1, \ldots, n\}\right\} & \leq 2 \exp \left(\frac{-\left(\frac{n \epsilon}{4}\right)^{2}}{2 n F_{n}(t)}\right) \\
& \leq 2 \exp \left(\frac{-n \epsilon^{2}}{32}\right)
\end{aligned}
$$

Using EQUATION 1.5,

$$
\mathbb{P}\left\{\sup _{t \in \mathbb{R}}\left|F_{n}^{s}\right|>\frac{\epsilon}{4} \mid X_{i}=x_{i} ; i \in\{1, \ldots, n\}\right\} \leq 2 n \exp \left(\frac{-n \epsilon^{2}}{32}\right)
$$

Noting that this does not depend on $X_{i}$ and using Equation 1.4

$$
\mathbb{P}\left\{\sup _{t \in \mathbb{R}}\left|F_{n}(t)-F(t)\right|>\epsilon\right\} \leq 8 n \exp \left(\frac{-n \epsilon^{2}}{32}\right)
$$




$$
\Rightarrow \sum_{n=1}^{\infty} \mathbb{P}\left\{\sup _{t \in \mathbb{R}}\left|F_{n}(t)-F(t)\right|>\epsilon\right\}<\infty \quad \forall \epsilon>0
$$

The second proof follows from an application of the Borel-Cantelli lemma.

\subsubsection{UNIFORM EMPIRICAL PROCESSES}

The motivation for this section is to transform $X_{i}$ onto the unit interval. If $X_{i}$ has a continuous distribution function $F$, then we can transform $X_{i}$ onto the unit interval in such a way that it has the same distribution as any other continuous distribution transformed in the same way. We call the transformed process the uniform empirical process. This allows us to derive limit theorems for the uniform empirical process, and then apply the limit theorem to any empirical process with continuous underlying distribution $F$.

Definition 1.1.7: Consider a transformation onto the closed unit interval $[0,1]$ as $\xi=\Gamma(t)$ for $t \in \mathbb{R}$. The corresponding empirical distribution function at stage $n \in \mathbb{N}$ is

$$
\Gamma_{n}(t)=\frac{1}{n} \#\left\{1 \leq i \leq n: \xi_{i} \leq t\right\} ; \quad t \in[0,1]
$$

where $\xi_{i}=\Gamma\left(X_{i}\right)$.

Definition 1.1.8: The reduced empirical process is defined as

$$
U_{n, \Gamma}(t)=n^{\frac{1}{2}}\left(\Gamma_{n}(t)-\Gamma(t)\right) ; \quad t \in[0,1]
$$

This is the empirical process of the random variables $\left\{\xi_{i}\right\}_{i=1}^{n}=\left\{\Gamma\left(X_{i}\right)\right\}_{i=1}^{n}$

Lemma 1.1.9: Let $\xi_{i}=F\left(X_{i}\right)$. If $F$ is continuous then

$$
\mathcal{L}\left(\xi_{i}\right)=\mathcal{U}(0,1)
$$


where $\mathcal{U}(0,1)$ is the law of the uniform distribution on $(0,1)$.

Proof: For $0 \leq t \leq 1$ :

$$
\begin{aligned}
\mathbb{P}\left\{\xi_{i} \leq t\right\} & =\mathbb{P}\left\{F\left(X_{i}\right) \leq t\right\} \\
& =\mathbb{P}\left\{X_{i} \leq F^{-1}(t)\right\} \\
& =F\left(F^{-1}(t)\right)=t=I(t)
\end{aligned}
$$

since $F$ is continuous, $F^{-1}$ is defined as $F^{-1}(t)=\inf \{x: F(x) \geq t\}$.

Definition 1.1.10: If $\Gamma=I$ then we denote $U_{n, I}=U_{n}$. This is called the uniform empirical process.

Theorem 1.1.11: For continuous $F$ we have

$$
\mathcal{L}\left(\sup _{x \in \mathbb{R}} n^{\frac{1}{2}}\left|F_{n}(x)-F(x)\right|\right)=\mathcal{L}\left(\sup _{t \in[0,1]}\left|U_{n}(t)\right|\right)
$$

Proof: By Lemma 1.1 .9 we know that $\xi_{1}, \ldots, \xi_{n}$ is a random sample of size $n$ from $\mathcal{U}(0,1)$

$$
\begin{aligned}
\sup _{x \in \mathbb{R}} n^{\frac{1}{2}}\left|F_{n}(x)-F(x)\right| & =\sup _{x \in \mathbb{R}} n^{\frac{1}{2}}\left|\frac{1}{n} \sum_{i=1}^{n} \mathbb{I}_{\left\{X_{i} \leq x\right\}}-F(x)\right| \\
& =\sup _{x \in \mathbb{R}} n^{\frac{1}{2}}\left|\frac{1}{n} \sum_{i=1}^{n} \mathbb{I}_{\left\{F\left(X_{i}\right) \leq F(x)\right\}}-F(x)\right| \\
& =\sup _{t \in[0,1]} n^{\frac{1}{2}}\left|\frac{1}{n} \sum_{i=1}^{n} \mathbb{I}_{\left\{\xi_{i} \leq t\right\}}-t\right| \\
& =\sup _{t \in[0,1]}\left|U_{n}(t)\right|
\end{aligned}
$$

The proof of the next theorem assumes some results which are proved later in the text. 


\section{TheOREM 1.1.12:}

$$
\mathbb{P}\left\{\sup _{t \in[0,1]}\left|U_{n}(t)\right| \leq x\right\} \rightarrow 1+2 \sum_{k=1}^{\infty}(-1)^{k} \exp \left(-2 k^{2} x^{2}\right)=H(x)
$$

Proof: We are going to assume that $U_{n}$ weakly converges to Brownian bridge, which is the statement of THEOREM 1.4.34. Given weak convergence all that remains is to show that $\sup _{t \in[0,1]}|\mathbb{U}(t)|$ has the distribution specified above, for $\mathbb{U}$ Brownian bridge. This follows directly from the statement of THEOREM 1.3.33.

\subsubsection{EMPIRICAL PROCESSES INDEXED BY FUNCTIONS}

The aim of this section is to give an introduction to a generalisation of the empirical process given in the previous section and to also generalise the Glivenko-Cantelli theorem. This subsection follows the beginning of Part 2 of VAN DER VAART \& WELLNER (1996).

The empirical process can be generalised by instead of considering not only indicator function on the real line, but considering collections of measurable functions mapping from the sample space into $\mathbb{R}$. So now $X_{i}$ are not necessarily in $\mathbb{R}$, but are instead in an arbitrary measurable space. We consider functions $f: \mathcal{X} \mapsto \mathbb{R}$ where $\mathcal{X}$ is an arbitrary measurable space.

Definition 1.1.13: The empirical measure $\mathbb{P}_{n}$ of a sample of random elements $X_{1}, X_{2}, \ldots, X_{n}$ in a measurable space $(\mathcal{X}, \mathcal{A})$ is the discrete random measure given by

$$
\mathbb{P}_{n}\{C\}=\frac{1}{n} \#\left\{1 \leq i \leq n: X_{i} \in C\right\}
$$

It can be described as the random measure that assigns mass $\frac{1}{n}$ at each observation. It can also be given as the linear combination of the Dirac 
measures at the observations

$$
\mathbb{P}_{n}=\frac{1}{n} \sum_{i=1}^{n} \delta_{X_{i}}
$$

where

$$
\delta_{X_{i}}(C)=\mathbb{I}_{\left\{X_{i} \in C\right\}}
$$

the indicator function that $X_{i}$ is an element of $C$.

Now consider a collection of measurable functions $\mathcal{F}$, where $f \in \mathcal{F}$ maps $\mathcal{X} \rightarrow \mathbb{R}$. The empirical measure induces a map from $\mathcal{F}$ to $\mathbb{R}$ given by

$$
f \mapsto \int f d \mathbb{P}_{n}=\mathbb{P}_{n} f
$$

Likewise the abbreviation $Q f=\int f d Q$ is used for a given measurable function $f$ and signed measure $Q$.

Definition 1.1.14: Let $\mathbb{P}$ be the common distribution of the $X_{i}$. The $\mathcal{F}$-indexed empirical process $\mathbb{G}_{n}$ is given by

$$
f \mapsto \mathbb{G}_{n} f=\sqrt{n}\left(\mathbb{P}_{n}-\mathbb{P}\right) f=\frac{1}{\sqrt{n}} \sum_{i=1}^{n}\left(f\left(X_{i}\right)-\int f d \mathbb{P}\right)
$$

We want to prove a generalisation of the Glivenko-Cantelli theorem for empirical processes indexed by functions. In the real case the (first) proof of the Glivenko-Cantelli theorem used the order on $\mathbb{R}$ to assert the existence of $t_{i}$ so $\mathbb{P}\left\{t_{i-1} \leq X_{i} \leq t_{i}\right\} \geq \frac{1}{m}$ for each $i=0,1, \ldots, m$ and $m \in \mathbb{N}$. No such ordering exists in general for a class of functions. We need an additional constraint on the size and the structure of the class, which is obtained using the following definitions.

Definition 1.1.15: A bracket $[l, u]$, where $l, u \in \mathcal{F}$, is the set of all functions $f \in \mathcal{F}: l \leq f \leq u$. 
Definition 1.1.16: An $\epsilon$-bracket is a bracket $[l, u]$ with $\|u-l\|_{\mathcal{F}}<\epsilon$

Definition 1.1.17: The bracketing number $N_{[]}(\epsilon, \mathcal{F},\|\cdot\|)$ is the minimum number of $\epsilon$-brackets needed to cover $\mathcal{F}$.

Definition 1.1.18: A Glivenko-Cantelli class has $\left\|\mathbb{P}_{n}-\mathbb{P}\right\|_{\mathcal{F}} \rightarrow 0$ in outer probability or outer almost surely, where $\|Q\|_{\mathcal{F}}=\sup \{|Q f|: f \in \mathcal{F}\}$.

For the rest of this subsection we are assuming that everything is appropriately measurable. For an overview of some of the measurability issues that can arise, see VAN DER VAART \& WELLNER (1996), among others.

Theorem 1.1.19: Let $\mathcal{F}$ be a class of measurable functions, such that for all $\epsilon>0, N_{[]}\left(\epsilon, \mathcal{F}, L_{1}(\mathbb{P})\right)<\infty$. Then $\mathcal{F}$ is Glivenko-Cantelli.

Proof: Fix $\epsilon>0$. As $N_{[]}\left(\epsilon, \mathcal{F}, L_{1}(\mathbb{P})\right)<\infty$ we can choose finitely many $\epsilon$-brackets $\left[l_{i}, u_{i}\right]$ with $\mathcal{F} \subseteq \bigcup_{i=1}^{n}\left[l_{i}, u_{i}\right]$ and $\mathbb{P} u_{i}-\mathbb{P} l_{i}<\epsilon$ for all $i$. For every $f \in \mathcal{F}$ there is a bracket such that

$$
\begin{array}{rlrl}
\left(\mathbb{P}_{n}-\mathbb{P}\right) f & \leq \mathbb{P}_{n} u_{i}-\mathbb{P} f & & \left(\text { As } u_{i} \geq f\right) \\
& =\left(\mathbb{P}_{n}-\mathbb{P}\right) u_{i}+\mathbb{P}\left(u_{i}-f\right) & \\
& \leq\left(\mathbb{P}_{n}-\mathbb{P}\right) u_{i}+\epsilon & \left(\text { As } f \in\left[l_{i}, u_{i}\right]\right)
\end{array}
$$

Therefore

$$
\sup _{f \in \mathcal{F}}\left(\mathbb{P}_{n}-\mathbb{P}\right) f \leq \max _{1 \leq i \leq n}\left(\mathbb{P}_{n}-\mathbb{P}\right) u_{i}+\epsilon
$$

Similarly

$$
\begin{array}{rlrl}
\left(\mathbb{P}_{n}-\mathbb{P}\right) f & \geq \mathbb{P}_{n} l_{i}-\mathbb{P} f & & \left(\text { As } l_{i} \leq f\right) \\
& =\left(\mathbb{P}_{n}-\mathbb{P}\right) l_{i}+\mathbb{P}\left(l_{i}-f\right) & \\
& \geq\left(\mathbb{P}_{n}-\mathbb{P}\right) l_{i}-\epsilon & \left(\text { As } f \in\left[l_{i}, u_{i}\right]\right)
\end{array}
$$


Therefore

$$
\inf _{f \in \mathcal{F}}\left(\mathbb{P}_{n}-\mathbb{P}\right) f \geq \min _{1 \leq i \leq n}\left(\mathbb{P}_{n}-\mathbb{P}\right) l_{i}-\epsilon
$$

Using the strong law of large numbers gives

$$
\limsup _{n \rightarrow \infty}\left\|\mathbb{P}_{n}-\mathbb{P}\right\|_{\mathcal{F}} \leq \epsilon
$$

almost surely for all $\epsilon>0$. Take a sequence of $\epsilon_{m} \downarrow 0$ to see that

$$
\limsup _{n \rightarrow \infty}\left\|\mathbb{P}_{n}-\mathbb{P}\right\|_{\mathcal{F}} \rightarrow 0
$$

almost surely. Therefore $\mathcal{F}$ is Glivenko-Cantelli.

\subsubsection{MULTI-DIMENSIONAL EMPIRICAL PROCESS RESULTS}

This subsection considers inequalities related to the uniform empirical process, which can be used to prove results like the law of iterated logarithms for empirical processes and probability inequalities about the local fluctuation of the uniform empirical process. Most of the results in this subsection are given without proof, though the proofs can be found in EinMahL (1993), among others.

Assume for this section that vectors take values in $(0,1)^{d} ; d \in \mathbb{N}$. The notation for coordinates of a vector $t$ is $t=\left\langle t_{1}, \ldots, t_{d}\right\rangle=\left\langle t_{j}\right\rangle$ and if $t_{j}=u$ for all $1 \leq j \leq d$ we simply write $\langle u\rangle$. For $s, t \in \mathbb{R}^{d}$ define $s \leq t$ as $s_{j} \leq t_{j}$ for all $1 \leq j \leq d$ and $s<t$ as $s_{j}<t_{j}$ for all $1 \leq j \leq d$.

Definition 1.1.20: Let $d$ be a fixed positive constant and fix $v \in \mathbb{N}^{d}$. For $t \in \mathbb{N}^{d}$ with $t \leq v$, let $Y_{t}$ be a random variable with mean 0 and finite variance $\sigma_{t}^{2}$ and assume these random variables are independent. For arbitrary $s, u \in \mathbb{N}^{d}$ 
with $s \leq u \leq v$ define partial sums by

$$
S_{u}=\sum_{t \leq u} Y_{t} \quad S_{s, u}=\sum_{s \leq t \leq v} Y_{t}
$$

and write $\sigma^{2}=\operatorname{Var}\left(S_{v}\right)=\sum_{t \leq v} \sigma_{t}^{2}$

TheORem 1.1.21: For all $\lambda \in \mathbb{R}$

$$
\mathbb{P}\left\{\max _{u \leq v} S_{u} \geq \lambda\right\} \leq 2^{d} \mathbb{P}\left\{S_{v} \geq \lambda-d \sqrt{2 \sigma^{2}}\right\}
$$

Proof: Let

$$
A=\left\{\max _{u \leq v} S_{u} \geq \lambda\right\}
$$

and

$$
B=\left\{\max _{u \leq v ; u_{d}=v_{d}} S_{u} \geq \lambda-\sqrt{2 \sigma^{2}}\right\}
$$

The first step is to prove

$$
\mathbb{P}\{A\} \leq 2 \mathbb{P}\{B\}
$$

Let

$$
C=\left\{k \in \mathbb{N}: k \leq \prod_{j=1}^{d} v_{j}\right\}
$$

be an index set for the possible vectors $u \leq v$ and let

$$
D=\left\{t \in \mathbb{N}^{d}: t_{j} \leq v_{j} ; \forall 1 \leq j \leq d\right\}
$$

be the set of possible vectors $u \leq v$. Furthermore let $g: C \rightarrow D$ be a fixed bijective function with the property that $k<l$ implies $(g(k))_{d} \leq(g(l))_{d}$. Write

$$
\widetilde{g(k)}=\left\langle(g(k))_{1},(g(k))_{2}, \ldots,(g(k))_{d-1}, v_{d}\right\rangle
$$

which can be seen as a projection of $g(k)$, replacing the last coordinate of $g(k)$ with the last coordinate of $v$. Also consider elementary events

$$
E_{k}=\left\{S_{g(k)} \geq \lambda\right\}
$$


and the events

$$
A_{k}=E_{k} \cup\left(\bigcup_{i=1}^{k-1} E_{i}^{c}\right),
$$

which are the events that $S_{g(k)}$ is the only vector from indices 1 to $k$ that is greater than $\lambda$. It is seen that $A_{k}$ are pairwise disjoint and $A=\bigcup_{k \in C} A_{k}$. We see by Chebyshev's inequality that

$$
\begin{aligned}
\mathbb{P}\left\{S_{\overparen{g(k)}}-S_{g(k)} \geq-\sqrt{2 \sigma^{2}}\right\} & \geq 1-\frac{E\left[\left(S_{\overparen{g(k)}}-S_{g(k)}\right)^{2}\right]}{2 \sigma^{2}} \\
& \geq 1-\frac{\operatorname{Var}\left(S_{\widetilde{g(k)}}\right)}{2 \sigma^{2}} \\
& \geq 1-\frac{\sigma^{2}}{2 \sigma^{2}}=\frac{1}{2}
\end{aligned}
$$

This implies that

$$
\mathbb{P}\left\{A_{k}\right\} \leq 2 \mathbb{P}\left\{A_{k}\right\} \mathbb{P}\left\{S_{\widetilde{g(k)}}-S_{g(k)} \geq-\sqrt{2 \sigma^{2}}\right\}
$$

Using the independence of $A_{k}$ and $\left\{S_{\widetilde{g(k)}}-S_{g(k)} \geq-\sqrt{2 \sigma^{2}}\right\}$

$$
\begin{aligned}
& =2 \mathbb{P}\left\{A_{k} \bigcap\left\{S_{\widetilde{g(k)}}-S_{g(k)} \geq-\sqrt{2 \sigma^{2}}\right\}\right\} \\
& \leq 2 \mathbb{P}\left\{A_{k} \bigcap\left\{S_{\widetilde{g(k)}} \geq \lambda-\sqrt{2 \sigma^{2}}\right\}\right\} \\
& =2 \mathbb{P}\left\{A_{k} \bigcap B\right\}
\end{aligned}
$$

This implies

$$
\begin{aligned}
\mathbb{P}\{A\} & =\sum_{k \in C} \mathbb{P}\left\{A_{k}\right\} \\
& \leq 2 \sum_{k \in C} \mathbb{P}\left\{A_{k} B\right\} \\
& =2 \mathbb{P}\{A B\} \\
& \leq 2 \mathbb{P}\{B\}
\end{aligned}
$$


This allows us to prove the result using induction. Letting $d=1$, the base case is proved by a direct application of the above result. Under the induction hypothesis we can say it holds for

$$
\mathbb{P}\left\{\max _{u \leq v} S_{u} \geq \lambda\right\} \leq 2^{k} \mathbb{P}\left\{S_{v} \geq \lambda-k \sqrt{2 \sigma^{2}}\right\}
$$

and need to prove that it holds for

$$
\mathbb{P}\left\{\max _{u \leq v} S_{u} \geq \lambda\right\} \leq 2^{k+1} \mathbb{P}\left\{S_{v} \geq \lambda-(k+1) \sqrt{2 \sigma^{2}}\right\}
$$

where all dimensions increase in size by 1 . This leads to

$$
\begin{aligned}
\mathbb{P}\left\{\max _{u \leq v} S_{u} \geq \lambda\right\} & \leq 2 \mathbb{P}\left\{\max _{u \leq v ; u_{d}=v_{d}} S_{u} \geq \lambda-\sqrt{2 \sigma^{2}}\right\} \\
& \leq 2\left(2^{k} \mathbb{P}\left\{\max _{u \leq v ; u_{d}=v_{d}} S_{u} \geq \lambda-k \sqrt{2 \sigma^{2}}-\sqrt{2 \sigma^{2}}\right\}\right) \\
& \leq 2^{k+1} \mathbb{P}\left\{S_{v} \geq \lambda-(k+1) \sqrt{2 \sigma^{2}}\right\}
\end{aligned}
$$

Definition 1.1.22: Let $N_{n}$ be a Poisson process on $[0,1]^{d}$, with $E\left[N_{n}(t)\right]=$ $n \Gamma(t)$ for all $t \in[0,1]^{d}$. Then

$$
Z_{n}(t)=\frac{1}{\sqrt{n}}\left(N_{n}(t)-n \Gamma(t)\right) \quad t \in[0,1]^{d}
$$

The similarity between the above definition and DEFInITION 1.1.8 should be apparent. This makes sense as conditional on $N_{n}(\langle 1\rangle)=n$, the processes $U_{n, \Gamma}$ and $Z_{n}$ are equal in law.

Definition 1.1.23: The function $\psi:[-1, \infty) \mapsto[0, \infty)$ is defined as

$$
\begin{aligned}
\psi(\lambda) & =\frac{2}{\lambda^{2}} \int_{0}^{\lambda} \log (1+\sigma) d \sigma \\
& = \begin{cases}2 \lambda^{-2}[(1+\lambda) \log (1+\lambda)-\lambda] & -1<\lambda<0 \text { or } 0<\lambda \\
2 & \lambda=-1 \\
1 & \lambda=0\end{cases}
\end{aligned}
$$


Result 1.1.24: For $\psi$

- $\psi$ is decreasing and continuous.

- $\psi(\lambda) \rightarrow \frac{2 \log (\lambda)}{\lambda}$ as $\lambda \rightarrow \infty$

- $\psi(\lambda) \geq \frac{1}{1+\frac{\lambda}{3}}$ for $\lambda \geq-1$

Definition 1.1.25: For the following two types of index sets are used.

(a) Quadrants of the form $\left[0, t_{1}\right] \times \ldots \times\left[0, t_{d}\right] ; t=\left\langle t_{j}\right\rangle \in[0,1]^{d}$

(b) Half-open rectangles of the form $\left(s_{1}, t_{i}\right] \times \ldots \times\left(s_{d}, t_{d}\right] ; s=\left\langle s_{j}\right\rangle<t=$ $\left\langle t_{j}\right\rangle ; s, t \in[0,1]^{d}$. Call the class of all such rectangles $\mathcal{R}$

RESUlT 1.1.26: For any $R \in \mathcal{R}$ with $\mathbb{P}\{\mathcal{R}\} \leq \frac{1}{2}$, all $\lambda \in \mathbb{R}$ and either choice of sign

$$
\begin{aligned}
\mathbb{P}\left\{\sup _{S \subset R} \pm U_{n, \Gamma}(S) \geq \lambda\right\} & \leq 2 \mathbb{P}\left\{\sup _{S \subset R} \pm Z_{n}(S) \geq \lambda\right\} \\
& \leq 2^{2 d+1} \mathbb{P}\left\{ \pm Z_{n}(R) \geq \lambda-2 d \sqrt{2 \mathbb{P}\{R\}}\right\}
\end{aligned}
$$

Lemma 1.1.27: Let $\tau>0$. For a $\operatorname{Poisson}(\tau)$ random variable $\mathrm{Z}$ and either choice of sign

$$
\mathbb{P}\{ \pm(Z-\tau) \geq \lambda\} \leq \exp \left(\frac{-\lambda^{2}}{2 \tau} \psi\left(\frac{ \pm \lambda}{\tau}\right)\right) ; \quad \lambda \geq 0
$$

For the minus part the additional assumption of $\lambda \leq \tau$ is required by the definition of $\psi$.

Proof: If $\lambda=0$, then this inequality is trivially true for either choice of sign. The other boundary case is for the negative choice of sign when $\lambda=\tau$, which 
is also trivially true. For all other cases, apply the Markov inequality

$$
\begin{aligned}
\mathbb{P}\{ \pm(Z-\tau) \geq \lambda\} & =\mathbb{P}\{\exp ( \pm s(Z-\tau)) \geq \exp (\lambda s)\} \\
& \leq \exp (-\lambda s) E[\exp ( \pm s(Z-\tau))] \\
& \leq \exp (-s(\lambda \pm \tau)) \exp (\tau(\exp ( \pm s)-1))
\end{aligned}
$$

The value of $s$ which minimizes this expression is $s= \pm \log \left(1 \pm \frac{\lambda}{\tau}\right)$, therefore

$$
\begin{aligned}
\mathbb{P}\{ \pm(Z-\tau) \geq \lambda\} & \leq \exp \left(-(\tau \pm \lambda) \log \left(1 \pm \frac{\lambda}{\tau}\right) \mp \lambda\right) \\
& =\exp \left(\frac{-\lambda^{2}}{2 \tau} \psi\left(\frac{ \pm \lambda}{\tau}\right)\right)
\end{aligned}
$$

Theorem 1.1.28: Let $n \in \mathbb{N}$ and $R \in \mathcal{R}$ with $0<\mathbb{P}\{R\} \leq \frac{1}{2}$. Then for any $\epsilon \in(0,1)$,

(a) For $\lambda \geq 0$;

$$
\mathbb{P}\left\{\sup _{S \subset R} U_{n, \Gamma}(S) \geq \lambda\right\} \leq C^{+} \exp \left(\frac{-(1-\epsilon) \lambda^{2}}{2 \mathbb{P}\{R\}} \psi\left(\frac{\lambda}{\sqrt{n} \mathbb{P}\{R\}}\right)\right) ;
$$

(b) For $0 \leq \lambda \leq \sqrt{n} \mathbb{P}\{R\}$;

$$
\mathbb{P}\left\{\sup _{S \subset R}-U_{n, \Gamma}(S) \geq \lambda\right\} \leq C^{-} \exp \left(\frac{-(1-\epsilon) \lambda^{2}}{2 \mathbb{P}\{R\}} \psi\left(\frac{-(1-\epsilon) \lambda}{\sqrt{n} \mathbb{P}\{R\}}\right)\right)
$$

(c) For $\lambda \geq 0$;

$$
\mathbb{P}\left\{\sup _{S \subset R}\left|U_{n, \Gamma}(S)\right| \geq \lambda\right\} \leq C \exp \left(\frac{-(1-\epsilon) \lambda^{2}}{2 \mathbb{P}\{R\}} \psi\left(\frac{\lambda}{\sqrt{n} \mathbb{P}\{R\}}\right)\right)
$$

where $S \in \mathcal{R}$ and $C^{+}, C^{-}$and $C \in(0, \infty)$ depend only on $d$ and $\epsilon$. 
Proof: Using Result 1.1.26 and Lemma 1.1.27 and $\lambda \geq \frac{4 d \sqrt{2 \mathbb{P}\{R\}}}{\epsilon}$

$$
\begin{aligned}
& \mathbb{P}\left\{\sup _{S \subset R} \pm U_{n, \Gamma}(S) \geq \lambda\right\} \\
& \leq 2^{2 d+1} \mathbb{P}\left\{ \pm Z_{n}(R) \geq \lambda-2 d \sqrt{2 \mathbb{P}\{R\}}\right\} \\
& =2^{2 d+1} \mathbb{P}\left\{ \pm\left(N_{n}(t)-n \mathbb{P}\{R\}\right) \geq \sqrt{n}(\lambda-2 d \sqrt{2 \mathbb{P}\{R\}})\right\} \\
& \left.\leq 2^{2 d+1} \exp \left(\frac{-n(\lambda-2 d \sqrt{2 \mathbb{P}\{R\}})^{2}}{2 n \mathbb{P}\{R\}} \psi \frac{ \pm \sqrt{n}(\lambda-2 d \sqrt{2 \mathbb{P}\{R\})}}{n \mathbb{P}\{R\}}\right)\right) \\
& \leq 2^{2 d+1} \exp \left(\frac{-\lambda^{2}\left(1-\frac{\epsilon}{2}\right)^{2}}{2 \mathbb{P}\{R\}} \psi\left(\frac{ \pm \lambda\left(1-\frac{\epsilon}{2}\right)}{\sqrt{n} \mathbb{P}\{R\}}\right)\right) \\
& \leq 2^{2 d+1} \exp \left(\frac{-\lambda^{2}(1-\epsilon)^{2}}{2 \mathbb{P}\{R\}} \psi\left(\frac{ \pm \lambda\left(1-\frac{\epsilon}{2}\right)}{\sqrt{n} \mathbb{P}\{R\}}\right)\right)
\end{aligned}
$$

As $\psi$ is decreasing

$$
\begin{gathered}
\psi\left(\frac{\lambda\left(1-\frac{\epsilon}{2}\right)}{\sqrt{n} \mathbb{P}\{R\}}\right) \geq \psi\left(\frac{\lambda}{\sqrt{n} \mathbb{P}\{R\}}\right) \\
\exp \left(\frac{-\lambda^{2}(1-\epsilon)^{2}}{2 \mathbb{P}\{R\}} \psi\left(\frac{\lambda\left(1-\frac{\epsilon}{2}\right)}{\sqrt{n} \mathbb{P}\{R\}}\right)\right) \leq \exp \left(\frac{-\lambda^{2}(1-\epsilon)^{2}}{2 \mathbb{P}\{R\}} \psi\left(\frac{\lambda}{\sqrt{n} \mathbb{P}\{R\}}\right)\right)
\end{gathered}
$$


which immediately gives (a). Furthermore

$$
\begin{gathered}
\psi\left(\frac{-\lambda\left(1-\frac{\epsilon}{2}\right)}{\sqrt{n} \mathbb{P}\{R\}}\right) \geq \psi\left(\frac{-\lambda(1-\epsilon)}{\sqrt{n} \mathbb{P}\{R\}}\right) \\
\exp \left(\frac{-\lambda^{2}(1-\epsilon)^{2}}{2 \mathbb{P}\{R\}} \psi\left(\frac{-\lambda\left(1-\frac{\epsilon}{2}\right)}{\sqrt{n} \mathbb{P}\{R\}}\right)\right) \leq \exp \left(\frac{-\lambda^{2}(1-\epsilon)^{2}}{2 \mathbb{P}\{R\}} \psi\left(\frac{-\lambda(1-\epsilon)}{\sqrt{n} \mathbb{P}\{R\}}\right)\right)
\end{gathered}
$$

which gives (b). (c) is a consequence of (a) and (b).

We finish this subsection by considering an inequality for the modulus of continuity (see Definition 1.2.12) for the uniform empirical process in $\mathbb{R}$.

Theorem 1.1.29: Let $0<\epsilon \leq \frac{1}{2}$ and $0<\alpha<\frac{1}{4}$. For $\lambda \geq 0$,

$$
\mathbb{P}\left\{\omega_{n}(\alpha) \geq \lambda \alpha^{\frac{1}{2}}\right\} \leq \frac{C}{\alpha} \exp \left(\frac{-(1-\epsilon) \lambda^{2}}{2} \psi\left(\frac{\lambda}{(n \alpha)^{\frac{1}{2}}}\right)\right)
$$

where $\omega_{n}(\alpha)$ is the modulus of continuity of the uniform empirical process.

Proof: Can be found in Einmahl (1993).

\subsection{LAW OF THE ITERATED LOGARITHM AND MODULUS OF CONTINUITY}

This section outlines the classical versions of the law of the iterated logarithm for Brownian motion, which describes the maximum fluctuation of Brownian motion, and a similar result for the modulus of continuity for Brownian motion, which describes the maximum local fluctuation of Brownian motion. As the limiting process of the uniform empirical process is Brownian bridge, we would expect that these results generalise to the uniform empirical process, and the generalisations are given at the end of the appropriate subsection. 


\subsubsection{LAW OF THE ITERATED LOGARITHM}

This section describes the law of the iterated logarithm, which gives the order of the maximum fluctuation of an empirical process. This lemma is from FELLER (1971a), among others.

Lemma 1.2.1: Let $\varphi(x)$ and $\Phi(x)$ be the density and distribution function of the standard normal distribution respectively. Then for $x>0$

$$
\left(\frac{1}{x}-\frac{1}{x^{3}}\right) \varphi(x) \leq 1-\Phi(x) \leq \frac{1}{x} \varphi(x)
$$

Proof: Using integration by parts

$$
\begin{aligned}
1-\Phi(x) & =\int_{x}^{\infty} \frac{1}{t}(t \varphi(t)) d t \\
& =\left.\frac{-1}{t} \varphi(t)\right|_{x} ^{\infty}-\int_{x}^{\infty} \frac{1}{t^{2}} \varphi(t) d t \\
& =\frac{1}{x} \varphi(x)-\int_{x}^{\infty} \frac{1}{t^{2}} \varphi(t) d t
\end{aligned}
$$

For the upper bound, as $x>0$ it follows $\int_{x}^{\infty} \frac{1}{t^{2}} \varphi(t) d t>0$, therefore

$$
1-\Phi(x) \leq \frac{1}{x} \varphi(x)
$$

For the lower bound, as $x>0$ and $\frac{1}{t^{2}} \downarrow$ as $t \uparrow$

$$
\begin{aligned}
1-\Phi(x) & \geq \frac{1}{x} \varphi(x)-\frac{1}{x^{2}} \int_{x}^{\infty} \varphi(t) d t \\
& =\frac{1}{x} \varphi(x)-\frac{1}{x^{2}}(1-\Phi(x)) \\
& \geq \frac{1}{x} \varphi(x)-\frac{1}{x^{2}} \frac{1}{x} \varphi(x)=\left(\frac{1}{x}-\frac{1}{x^{3}}\right) \varphi(x)
\end{aligned}
$$


Corollary 1.2.2: As $x \rightarrow \infty$,

$$
1-\Phi(x) \sim \frac{1}{x} \varphi(x)
$$

\section{Proof:}

$$
\frac{1}{x}-\frac{1}{x^{3}} \rightarrow \frac{1}{x}
$$

as $x \rightarrow \infty$. Using LEMma 1.2.1 the result follows.

The following theorem is from Ito \& MCKeAn JR (1974), among others. Consider $h(t) \in C(0,1]: h(t) \geq 0$, a non-negative continuous function on the interval $(0,1]$. Using Blumenthal's 0-1 law implies that $\mathbb{P}\left\{\limsup _{t \downarrow 0} \frac{\mathbb{S}(t)}{h(t)}<1\right\} \in$ $\{0,1\}$, where $\mathbb{S}(t)$ is Brownian motion (see Defintion 1.3.1).

Definition 1.2.3: $h(t)$ belongs to the upper class if $\mathbb{P}\left\{\limsup _{t \downarrow 0} \frac{\mathbb{S}(t)}{h(t)}<1\right\}=1$. $h(t)$ belongs to the lower class if $\mathbb{P}\left\{\limsup _{t \downarrow 0} \frac{\mathbb{S}(t)}{h(t)}<1\right\}=0$.

Lemma 1.2.4 (Kolmogorov's Test): If $h(t) \uparrow$ and $t^{-\frac{1}{2}} h(t) \downarrow$ for small $t>0$, then $h(t)$ belongs to the upper class if

$$
\int_{0}^{b} t^{-\frac{3}{2}} h(t) \exp \left(-\frac{h^{2}(t)}{2 t}\right) d t=\int_{0^{+}} t^{-\frac{3}{2}} h(t) \exp \left(-\frac{h^{2}(t)}{2 t}\right) d t
$$

converges for some $b>0$.

Proof: Define $\mathbb{P}_{0}$ to be the distribution of the Brownian motion which has initial point 0 . Let $T_{a} \leq t$ be the first passage time of level $a$ for Brownian motion. It will be shown in THEOREM 1.3.28 that the first passage time distribution is

$$
\mathbb{P}_{0}\left\{T_{a} \leq t\right\}=\int_{0}^{t} \frac{a}{\sqrt{2 \pi s^{3}}} \exp \left(-\frac{a^{2}}{2 s}\right) d s
$$

Let $0<a=t_{1}<t_{2}<\cdots<t_{n}=b \leq 1$ be increments of $(0,1]$ such that $h(t) \uparrow$ for $t \leq b$. Then, from Figure 1.1, 


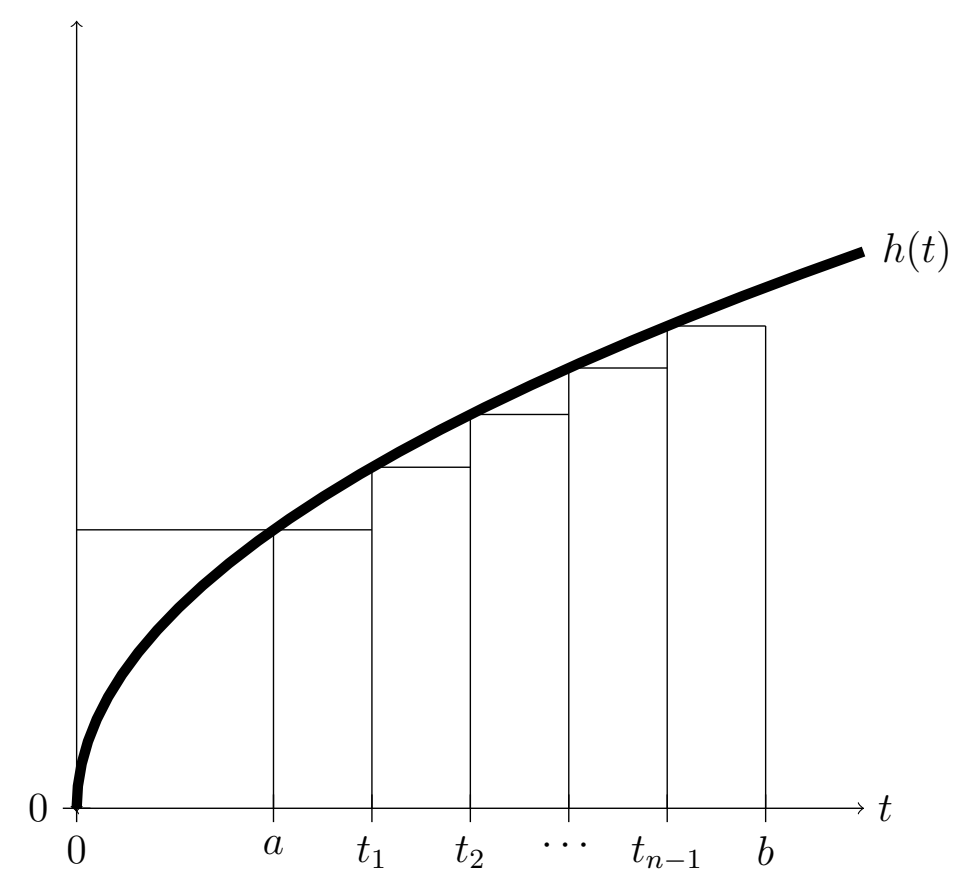

FiguRE 1.1: If $\mathbb{S}(t)$ exceeds $h(t)$ on $(a, b]$, then it must have already exceeded the stepwise approximation shown. 


$$
\begin{aligned}
\mathbb{P}_{0} & \{\mathbb{S}(t) \geq h(t) \text { for some } a \leq t \leq b\} \\
& \leq \mathbb{P}_{0}\left\{T_{h(a)} \leq a\right\}+\sum_{m \geq 2} \mathbb{P}_{0}\left\{t_{m-1}<T_{h\left(t_{m-1}\right)} \leq t_{m}\right\} \\
& =\int_{0}^{a} \frac{h(a)}{\sqrt{2 \pi t^{3}}} \exp \left(-\frac{h^{2}(a)}{2 t}\right) d t+\sum_{m \geq 2} \int_{t_{m-1}}^{t_{m}} \frac{h\left(t_{m-1}\right)}{\sqrt{2 \pi t^{3}}} \exp \left(-\frac{h^{2}\left(t_{m-1}\right)}{2 t}\right) d t
\end{aligned}
$$

As $\max \left\{\left|t_{m}-t_{m-1}\right|\right\} \downarrow 0$ as $n \rightarrow \infty$ this becomes

$$
\leq \int_{0}^{\frac{a}{h^{2}(a)}} \frac{\exp \left(-\frac{1}{2 t}\right) d t}{\sqrt{2 \pi t^{3}}}+\int_{a}^{b} \frac{h(t)}{\sqrt{2 \pi t^{3}}} \exp \left(-\frac{h^{2}(t)}{2 t}\right) d t
$$

At this stage we need to show that $\lim _{t \downarrow 0} t^{-\frac{1}{2}} h(t)=\infty$. Assume that $\lim _{t \downarrow 0} t^{-\frac{1}{2}} h(t) \rightarrow M$ for $M<\infty$. As $t^{-\frac{1}{2}} h(t) \downarrow$ and $h(t)$ is non-negative, it follows that $\lim _{t \downarrow 0} t^{-\frac{1}{2}} h(t) \neq 0$ unless $h(t)=0$, which is trivial. If $\lim _{t \downarrow 0} t^{-\frac{1}{2}} h(t) \rightarrow M<\infty$ for $M>0$, the integral

$$
\int_{0^{+}} t^{-\frac{3}{2}} h(t) \exp \left(-\frac{h^{2}(t)}{2 t}\right) d t \rightarrow \int_{0^{+}} \frac{M \exp \left(-\frac{M^{2}}{2}\right)}{t} d t=\infty
$$

which contradicts the assumption that $\int_{0^{+}} t^{-\frac{3}{2}} h(t) \exp \left(-\frac{h^{2}(t)}{2 t}\right) d t$ is finite. Therefore $\lim _{t \downarrow 0} t^{-\frac{1}{2}} h(t)=\infty$ and $\frac{a}{h^{2}(a)} \rightarrow 0$ as $a \rightarrow 0$. Letting $a \downarrow 0$ gives

$$
\mathbb{P}_{0}\{\mathbb{S}(t) \geq h(t) \text { for some } 0<t \leq b\} \leq \int_{0}^{b} \frac{h(t)}{\sqrt{2 \pi t^{3}}} \exp \left(-\frac{h^{2}(t)}{2 t}\right) d t \rightarrow 0
$$

as $b \downarrow 0$.

Theorem 1.2.5 (Local Law of the Iterated Logarithm):

$$
\mathbb{P}_{0}\left\{\limsup _{t \downarrow 0} \frac{\mathbb{S}(t)}{\sqrt{2 t \log \log \left(\frac{1}{t}\right)}}=1\right\}=1
$$


Proof: Consider the function $h(t)=(1+\epsilon) \sqrt{2 t \log \log \left(\frac{1}{t}\right)}$ for $\epsilon>0$. As $h(t)$ is positive, it is increasing if

$$
\begin{aligned}
\frac{d}{d t} t \log \log \left(\frac{1}{t}\right)= & \log \log \left(\frac{1}{t}\right)-\frac{1}{\log \left(\frac{1}{t}\right)}>0 \\
\Leftrightarrow \log \left(\frac{1}{t}\right) \log \log \left(\frac{1}{t}\right) & >1 \\
t & <e^{-1}
\end{aligned}
$$

Then $h(t)$ is increasing for $t<e^{-1}$ and $t^{-\frac{1}{2}} h(t)$ is decreasing for $t \leq 1$ as $\log \log \left(\frac{1}{t}\right)$ decreases as $t$ increases. We also need to show the convergence of $\int_{0^{+}} t^{-\frac{3}{2}} h(t) \exp \left(-\frac{h^{2}(t)}{2 t}\right) d t=\int_{0}^{\delta}(1+\epsilon) \sqrt{2 \log \log \left(\frac{1}{t}\right)}\left(\log \left(\frac{1}{t}\right)\right)^{-(1+\epsilon)^{2}} \frac{d t}{t}$ Letting $v=\log \log \left(\frac{1}{t}\right)$ gives

$$
\begin{aligned}
e^{v}=\log \left(\frac{1}{t}\right) \quad \frac{1}{t} & =\exp \left(e^{v}\right) \quad d t=-e^{v} \exp \left(-e^{v}\right) d v \\
& =\int_{\log \log \left(\frac{1}{\delta}\right)}^{\infty}(1+\epsilon) \sqrt{2 v}\left(e^{v}\right)^{1-(1+\epsilon)^{2}} d v .
\end{aligned}
$$

For $\epsilon>0$ we get convergence and for $-1<\epsilon<0$ the integral diverges, so it follows from LEMMA 1.2.4 that

$$
\mathbb{P}_{0}\left\{\limsup _{t \downarrow 0} \frac{\mathbb{S}(t)}{\sqrt{2 t \log \log \left(\frac{1}{t}\right)}} \leq 1\right\}=1
$$

Given $0<\epsilon<1$ and ignoring the set of Brownian paths for which 
$\limsup _{t \downarrow 0} \mathbb{S}(t)>\sqrt{2 t \log \log \left(\frac{1}{t}\right)}$, which has probability 0 , if

$$
A_{n}=\left\{B_{\epsilon^{n}}<(1-3 \sqrt{\epsilon}) \sqrt{2 \epsilon^{n} \log \log \epsilon^{-n}}\right\}
$$

occurs as $n \uparrow \infty$, then

$\mathbb{S}\left(\epsilon^{n}\right)-\mathbb{S}\left(\epsilon^{n+1}\right)<(1-3 \sqrt{\epsilon}) \sqrt{2 \epsilon^{n} \log \log \epsilon^{-n}}+\frac{3}{2} \sqrt{2 \epsilon^{n+1} \log \log \epsilon^{-n-1}}$ and $A_{n} \subseteq B_{n}$ as $n \rightarrow \infty$, where

$$
B_{n}=\left\{\mathbb{S}\left(\epsilon^{n}\right)-\mathbb{S}\left(\epsilon^{n+1}\right)<(1-\sqrt{\epsilon}) \sqrt{2 \epsilon^{n} \log \log \epsilon^{-n}}\right\}
$$

If $\theta=\frac{1-\sqrt{\epsilon}}{\sqrt{1-\epsilon}}<1$ and $c=\theta \sqrt{2 \log \log \epsilon^{-n}}$, then using COROLlaRY 1.2.2

$1-\mathbb{P}_{0}\left\{B_{n}\right\}=\int_{c}^{\infty} \varphi(b) d b \sim \frac{1}{\theta} \frac{1}{\log n+\log \log \epsilon^{-1}} \frac{1}{\sqrt{2 \pi}}\left(n^{-\theta^{2}}+\left(\log \left(\epsilon^{-1}\right)\right)^{-\theta^{2}}\right)$

so $1-\mathbb{P}_{0}\left\{B_{n}\right\}=C \frac{n^{-\theta^{2}}}{\sqrt{\log n}}$ where $C$ does not depend on $n$. Using Cauchy's condensation test

$$
\sum_{n=2}^{\infty} \frac{n^{-\theta^{2}}}{\sqrt{\log n}} \rightarrow \infty \Leftrightarrow \sum_{n=2}^{\infty} 2^{n} \frac{\left(2^{n}\right)^{-\theta^{2}}}{\sqrt{n \log 2}}=\sum_{n=2}^{\infty} \frac{\left(2^{n\left(1-\theta^{2}\right)}\right)}{\sqrt{n \log 2}} \rightarrow \infty
$$

which is true as $\theta^{2}<1$ implies $\frac{\left(2^{n\left(1-\theta^{2}\right)}\right)}{\sqrt{n \log 2}} \rightarrow \infty$ as $n \rightarrow \infty$. As $\sum_{n=2}^{\infty} \frac{n^{-\theta^{2}}}{\sqrt{\log n}}$ diverges and $B_{n}$ are independent, using the Borel-Cantelli lemma

$$
1-\mathbb{P}_{0}\left\{B_{n}\right\}=1 \Rightarrow \mathbb{P}_{0}\left\{B_{n}\right\}=0
$$

Therefore

$$
\begin{aligned}
\mathbb{P}_{0}\left\{\bigcup_{n \geq 1} \bigcap_{m \geq n} A_{m}\right\} & \leq \mathbb{P}_{0}\left\{\bigcup_{n \geq 1} \bigcap_{m \geq n} B_{m}\right\} \\
& =\lim _{n \uparrow \infty} \mathbb{P}_{0}\left\{\bigcap_{m \geq n} B_{m}\right\}=0
\end{aligned}
$$


This shows that

$$
\begin{aligned}
1 & =\mathbb{P}_{0}\left\{\mathbb{S}\left(\epsilon^{n}\right) \geq(1-3 \sqrt{\epsilon}) \sqrt{2 \epsilon^{n} \log \log \left(\epsilon^{-n}\right)}, \text { i.o., } n \uparrow \infty\right\} \\
& \leq \mathbb{P}_{0}\left\{\limsup _{t \downarrow 0} \frac{\mathbb{S}(t)}{\sqrt{2 \log \log \left(\frac{1}{t}\right)}} \geq 1-3 \sqrt{\epsilon}\right\}
\end{aligned}
$$

Letting $\epsilon \downarrow 0$ gives

$$
\mathbb{P}_{0}\left\{\limsup _{t \downarrow 0} \frac{\mathbb{S}(t)}{\sqrt{2 t \log \log \left(\frac{1}{t}\right)}} \geq 1\right\}=1
$$

This implies that

$$
\mathbb{P}_{0}\left\{\limsup _{t \downarrow 0} \frac{\mathbb{S}(t)}{\sqrt{2 t \log \log \left(\frac{1}{t}\right)}}=1\right\}=1
$$

REMARK 1.2.6: Kolmogorov's test also establishes that if

$$
\int_{0}^{b} t^{-\frac{3}{2}} h(t) \exp \left(-\frac{h^{2}(t)}{2 t}\right) d t=\int_{0^{+}} t^{-\frac{3}{2}} h(t) \exp \left(-\frac{h^{2}(t)}{2 t}\right) d t
$$

diverges for all $b>0$, then $h(t)$ must belong to the lower class. This is more difficult to prove than convergence implies upper class, but using Kolmogorov's test in this form means that proving the local law of the iterated logarithm is the same as showing

$$
h(t)=(1+\epsilon) \sqrt{2 t \log \log \left(\frac{1}{t}\right)} \quad \text { is upper class for } \epsilon>0
$$


and

$$
h(t)=(1-\epsilon) \sqrt{2 t \log \log \left(\frac{1}{t}\right)} \quad \text { is lower class for } \epsilon>0
$$

which was done in the proof given above.

Corollary 1.2.7: Let $\mathbb{U}$ be Brownian bridge. Then

$$
\mathbb{P}_{0}\left\{\limsup \frac{\mathbb{U}(t)}{\sqrt{2 t \log \log \left(\frac{1}{t}\right)}}=1\right\}=1
$$

Proof: Let $\mathbb{U}(t)=\mathbb{S}(t)-t \mathbb{S}(1)$. The result follows directly from TheOrem 1.2 .5

We conclude this subsection by considering law of the iterated logarithm results for empirical processes. The theorem is Smirnov's law of the iterated logarithm (see Smirnov (1944)), which will be proven using the following theorem.

Theorem 1.2.8: Let $\lambda_{n}$ be increasing. If $\sum_{n=1}^{\infty} \frac{\lambda_{n}^{2}}{n} \exp \left(-2 \lambda_{n}^{2}\right)<\infty$,

$$
\mathbb{P}\left\{\left\|\mathbb{U}_{n}\right\| \geq \lambda_{n} \text { i.o. }\right\}=0 .
$$

Alternatively, if $\sum_{n=1}^{\infty} \frac{\lambda_{n}^{2}}{n} \exp \left(-2 \lambda_{n}^{2}\right)=\infty$,

$$
\mathbb{P}\left\{\left\|\mathbb{U}_{n}\right\| \geq \lambda_{n} \text { i.o. }\right\}=1 \text {. }
$$

Proof: Refer to Chung (1949).

Theorem 1.2.9: Let $\mathbb{U}_{n}$ denote the uniform empirical process. Then

$$
\limsup _{n \rightarrow \infty} \frac{\left\|\mathbb{U}_{n}\right\|}{\sqrt{2 \log \log n}}=\frac{1}{2} \quad \text { almost surely }
$$


Proof: This proof follows Shorack \& Wellner (1986). The lower bound can be established by considering $\mathbb{U}_{n}\left(\frac{1}{2}\right)$, which is the average of $n$ independent Bernoulli $\left(\frac{1}{2}\right)$ random variables, centered by subtracting the expected value $\frac{1}{2}$. The law of iterated logarithm for Bernoulli variables (see FELLER (1971b), among others) implies that

$$
\limsup _{n \rightarrow \infty} \frac{\left\|\mathbb{U}_{n}\right\|}{\sqrt{2 \log \log n}} \geq \limsup _{n \rightarrow \infty} \frac{\mathbb{U}_{n}\left(\frac{1}{2}\right)}{\sqrt{2 \log \log n}} \geq \frac{1}{2} \quad \text { almost surely }
$$

For the upper bound let $\lambda_{n}=(1+\epsilon) \frac{\sqrt{2 \log \log (n)}}{2}$ for any $\epsilon>0$. We consider

$$
\begin{aligned}
\sum_{n=1}^{\infty} & \frac{\lambda_{n}^{2}}{n} \exp \left(-2 \lambda_{n}^{2}\right) \\
& =\sum_{n=1}^{\infty} \frac{(1+\epsilon)^{2}}{2} \frac{\log \log (n)}{n} \exp \left(-(1+\epsilon)^{2} \log \left((\log (n))^{-(1+\epsilon)^{2}}\right)\right) \\
& =\sum_{n=1}^{\infty} \frac{(1+\epsilon)^{2}}{2} \frac{\log \log (n)}{n(\log n)^{(1+\epsilon)^{2}}}
\end{aligned}
$$

Using the Cauchy condensation test, this will converge if and only if

$$
\sum_{n=1}^{\infty} \frac{(1+\epsilon)^{2}}{2} \frac{\log (n \log 2)}{n^{(1+\epsilon)^{2}}(\log 2)^{(1+\epsilon)^{2}}}
$$

converges, which converges if and only if

$$
\sum_{n=1}^{\infty} C n 2^{1-(1+\epsilon)^{2}}
$$

converges, for constant $C$ which does not depend on $n$, which is true for $\epsilon>0$. Using Theorem 1.2.8, it follows that

$$
\mathbb{P}\left\{\frac{\left\|\mathbb{U}_{n}\right\|}{\sqrt{2 \log \log n}}>\frac{1}{2}(1+\epsilon) \text { i.o. }\right\}=0
$$


for all $\epsilon>0$. Letting $\epsilon \downarrow 0$ completes the proof.

\subsubsection{LEVY MODULUS OF CONTINUITY}

Lemma 1.2.10 (Chung, Erdos, Sirao): If $h(t) \in C(0,1]$ is positive and if $h(t) \uparrow$ and $t^{-\frac{1}{2}} h(t) \downarrow$ as $t \downarrow 0$, then

$$
\mathbb{P}\left\{\lim _{\epsilon \downarrow 0} \sup _{t_{2}-t_{1}=\epsilon} \frac{\left|\mathbb{S}\left(t_{2}\right)-\mathbb{S}\left(t_{1}\right)\right|}{h(\epsilon)}<1 ; 0 \leq t_{1}<t_{2} \leq 1\right\}=1
$$

if for any $\delta>0$

$$
\int_{0+}^{\delta} t^{-\frac{7}{2}} h^{3}(t) \exp \left(-\frac{h^{2}(t)}{2 t}\right) d t<\infty
$$

and

$$
\mathbb{P}\left\{\lim _{\epsilon \downarrow 0} \sup _{t_{2}-t_{1}=\epsilon} \frac{\left|\mathbb{S}\left(t_{2}\right)-\mathbb{S}\left(t_{1}\right)\right|}{h(\epsilon)}<1 ; 0 \leq t_{1}<t_{2} \leq 1\right\}=0
$$

if for all $\delta>0$

$$
\int_{0+}^{\delta} t^{-\frac{7}{2}} h^{3}(t) \exp \left(-\frac{h^{2}(t)}{2 t}\right) d t=\infty
$$

Proof: The reader is referred to Chung et AL. (1959) for a proof of this result.

Using this lemma we intend to find a function $h(\epsilon)$ so that

$$
\mathbb{P}\left\{\lim _{\epsilon \downarrow 0} \sup _{t_{2}-t_{1}=\epsilon} \frac{\left|\mathbb{S}\left(t_{2}\right)-\mathbb{S}\left(t_{1}\right)\right|}{h(\epsilon)}=1\right\}=1 \quad 0 \leq t_{1}<t_{2} \leq 1
$$

As the variance of the increment $\mathbb{S}\left(t_{2}\right)-\mathbb{S}\left(t_{1}\right)$ is $\epsilon$ (if $t_{2}-t_{1}=\epsilon$ ), a reasonable first choice for the function $h(\epsilon)$ could be $h(\epsilon)=M \sqrt{\epsilon}$, for some constant $M$. For $M$ large enough we would initially expect that

$$
\mathbb{P}\left\{\lim _{\epsilon \downarrow 0} \sup _{t_{2}-t_{1}=\epsilon} \frac{\left|\mathbb{S}\left(t_{2}\right)-\mathbb{S}\left(t_{1}\right)\right|}{M \sqrt{\epsilon}} \leq 1\right\}=1 \quad 0 \leq t_{1}<t_{2} \leq 1
$$


because for fixed $\epsilon$, the probability of an increment of Brownian motion exceeding $M \sqrt{\epsilon} \rightarrow 0$ as $M \uparrow$. Using LEMma 1.2.10

$$
\int_{0+}^{\delta} t^{-\frac{7}{2}} M^{3} t^{\frac{3}{2}} \exp \left(-\frac{M^{2} t}{2 t}\right) d t=M^{3} \exp \left(-\frac{M^{2}}{2}\right) \int_{0+}^{\delta} t^{-2} d t=\infty
$$

so we conclude that

$$
\mathbb{P}\left\{\lim _{\epsilon \downarrow 0} \sup _{t_{2}-t_{1}=\epsilon} \frac{\left|\mathbb{S}\left(t_{2}\right)-\mathbb{S}\left(t_{1}\right)\right|}{M \sqrt{\epsilon}} \leq 1\right\}=0 \quad 0 \leq t_{1}<t_{2} \leq 1 .
$$

This means there will be infinitely many increments which cross $M \sqrt{\epsilon}$ as $\epsilon \rightarrow 0$. We need a different function for $h(\epsilon)$, which is given in the next theorem.

Theorem 1.2.11 (P. Levy's Holder condition):

$$
\mathbb{P}_{0}\left\{\lim _{\epsilon \downarrow 0} \sup _{t_{2}-t_{1}=\epsilon} \frac{\left|\mathbb{S}\left(t_{2}\right)-\mathbb{S}\left(t_{1}\right)\right|}{\sqrt{2 \epsilon \log \left(\frac{1}{\epsilon}\right)}}=1 ; 0 \leq t_{1}<t_{2} \leq 1\right\}=1
$$

Proof: Let $h(t)=(1+\epsilon) \sqrt{2 t \log \left(\frac{1}{t}\right)}$. We show equality in two parts.

Firstly by showing that the integral diverges for $\epsilon \leq 0$, so that the increments of Brownian motion cross $\sqrt{2 t \log \left(\frac{1}{t}\right)}$ infinitely often, which shows we have found a function that is not too large. Secondly by showing that for any $\epsilon>0$ the integral diverges, so if the function is increased by any small multiple, the increments of Brownian motion will no longer cross infinitely often. It is seen that $h(t) \uparrow$ for $t<e^{-1}, t^{-\frac{1}{2}} h(t) \downarrow$ for $t \leq 1$. Using LEMMA 1.2.10

$$
\begin{aligned}
\int_{0}^{\delta} t^{-\frac{7}{2}} h^{3}(t) \exp \left(-\frac{h^{2}(t)}{2 t}\right) d t & =2^{\frac{3}{2}}(1+\epsilon)^{3} \int_{0}^{\delta}\left(\log \left(\frac{1}{t}\right)\right)^{\frac{3}{2}} t^{(1+\epsilon)^{2}-2} d t \\
& =\int_{\log \left(\frac{1}{\delta}\right)}^{\infty} u^{\frac{3}{2}}\left(e^{u}\right)^{1-(1+\epsilon)^{2}} d u
\end{aligned}
$$

which diverges for small $\epsilon \leq 0$ and converges for $\epsilon>0$. 
We also want to show that other functions can also satisfy (1.7). Let

$$
h(t)=\sqrt{2 t\left[\log \left(\frac{1}{t}\right)+\frac{5}{2} \log _{2}\left(\frac{1}{t}\right)+\sum_{j=3}^{n-1} \log _{j}\left(\frac{1}{t}\right)+(1+\delta) \log _{n}\left(\frac{1}{t}\right)\right]}
$$

Then

$$
\begin{aligned}
-\frac{h^{2}(t)}{2 t}= & \log (t)+\log \left(\frac{1}{\left(\log \left(\frac{1}{t}\right)\right)^{\frac{5}{2}}}\right)+\sum_{i=2}^{n-2} \log \left(\frac{1}{\log _{i}\left(\frac{1}{t}\right)}\right) \\
& +\log \left(\frac{1}{\left(\log _{n-1}\left(\frac{1}{t}\right)\right)^{1+\delta}}\right)
\end{aligned}
$$

and

$$
\begin{aligned}
& \lim _{\epsilon \downarrow 0} \int_{0}^{\epsilon} t^{-\frac{7}{2}} h^{3}(t) \exp \left(-\frac{h^{2}(t)}{2 t}\right) d t \\
& =\lim _{\epsilon \downarrow 0} \int_{0}^{\epsilon} t^{-\frac{7}{2}} h^{3}(t) t \frac{1}{\left(\log \left(\frac{1}{t}\right)\right)^{\frac{5}{2}}} \frac{1}{\log _{2}\left(\frac{1}{t}\right)} \cdots \frac{1}{\log _{n-2}\left(\frac{1}{t}\right)} \frac{1}{\left(\log _{n-1}\left(\frac{1}{t}\right)\right)^{1+\delta}} d t
\end{aligned}
$$

Let $u=\log _{n}\left(\frac{1}{t}\right), d u=\frac{-1}{t} \frac{1}{\left(\log \left(\frac{1}{t}\right)\right)} \prod_{j=2}^{n-2} \frac{1}{\log _{j}\left(\frac{1}{t}\right)} \frac{1}{\left(\log _{n-1}\left(\frac{1}{t}\right)\right)} d t$ and $\frac{1}{t}=\exp _{n}(u)$. For $\epsilon$ small enough

$$
\begin{aligned}
& =\lim _{\epsilon \downarrow 0} \int_{\log _{n}\left(\frac{1}{\epsilon}\right)}^{\infty} \frac{\left(u(1+\delta)+\exp (u)+\ldots+\frac{5}{2} \exp _{n-1}(u)+\exp _{n-1}(u)\right)^{\frac{3}{2}} d u}{(\exp (u))^{\delta}\left(\exp _{n-1}(u)\right)^{\frac{3}{2}}} \\
& =\lim _{\epsilon \downarrow 0} \int_{\log _{n}}^{\infty}\left(\frac{1}{\epsilon}\right)\left(\frac{u(1+\delta)}{\exp _{n-1}(u)}+\cdots+1\right)^{\frac{3}{2}} \exp (-u \delta) d u
\end{aligned}
$$


which converges for $\delta>0$ and diverges for $\delta \leq 0$. Therefore, for $0 \leq t_{1}<t_{2} \leq 1$

$$
\mathbb{P}_{0}\left\{\lim _{\epsilon \downarrow 0} \sup _{t_{2}-t_{1}=\epsilon} \frac{\left|\mathbb{S}\left(t_{2}\right)-\mathbb{S}\left(t_{1}\right)\right|}{\sqrt{2 \epsilon\left[\log \left(\frac{1}{\epsilon}\right)+\frac{5}{2} \log _{2}\left(\frac{1}{\epsilon}\right)+\cdots+\log _{n}\left(\frac{1}{\epsilon}\right)\right]}}=1\right\}=1
$$

We finish this section by considering an extension of this type of results to the uniform empirical process.

Definition 1.2.12: The modulus of continuity $\omega_{n}$ of the uniform empirical process $\mathbb{U}_{n}$ is defined as

$$
\omega_{n}(a)=\sup _{0 \leq h \leq a} \sup _{0 \leq t \leq 1-h}\left|\mathbb{U}_{n}(t+h)-\mathbb{U}_{n}(t)\right|
$$

Theorem 1.2.13: Let $a_{n} \downarrow 0, n a_{n} \uparrow, \frac{\log \left(a_{n}^{-1}\right)}{\log \log (n)} \rightarrow \infty$ and $\frac{\log \left(a_{n}^{-1}\right)}{n a_{n}} \rightarrow 0$. Then

$$
\lim _{n \rightarrow \infty} \frac{\omega_{n}\left(a_{n}\right)}{\sqrt{2 a_{n} \log \log \left(\frac{1}{a_{n}}\right)}}=1 \quad \text { a.s. }
$$

Proof: Can be found in Shorack \& Wellner (1986), among others.

Other limiting behaviours are possible depending on the exact rate which $a_{n}$ converges to 0. An excellent reference for a discussion of this topic is SHORACK \& Wellner (1986).

\subsection{SOME LIMITING PROCESSES}

This section provides an introduction to the theory of Gaussian (or normal) processes, the natural limit of many empirical processes. The theory of Gaussian processes is of independent interest and has many applications 
beyond empirical processes, but will mainly serve to motivate the next section. The aim of this section is to give an overview of the theory behind some of the processes which can arise as limits of empirical processes. The following section follows SHORACK \& WELLNER (1986).

\subsubsection{GAUSSIAN PROCESSES}

Definition 1.3.1: Let $\mathbb{S}$ be a Gaussian process. Then if

1. $E[\mathbb{S}(t)]=0 ; \quad \forall t$

2. $\operatorname{Cov}\left[\mathbb{S}(t), \mathbb{S}\left(t^{\prime}\right)\right]=\min \left\{t, t^{\prime}\right\}=t \wedge t^{\prime} ; \quad \forall t, t^{\prime}$

$\mathbb{S}$ is called Brownian motion.

Definition 1.3.2: Let $T$ be an interval in $(-\infty, \infty)$. Then

$$
X(s, t] \equiv X(t)-X(s) ; \quad \forall s, t \in T
$$

is an increment of $X$.

Definition 1.3.3: If $X\left(t_{0}\right), X\left(t_{0}, t_{1}\right], \ldots, X\left(t_{k-1}, t_{k}\right]$ are independent random variables for all $k \geq 1$ and all $t_{0}<t_{1}<\ldots<t_{k}$ then $X$ has independent increments.

DeFinition 1.3.4: If

$$
\left(X\left(t_{1}+h\right), X\left(t_{2}+h\right), \ldots, X\left(t_{k}+h\right)\right) \cong\left(X\left(t_{1}\right), X\left(t_{2}\right), \ldots, X\left(t_{k}\right)\right)
$$

for all $k \geq 1, h \geq 0$ and all time points in $T$, then $X$ is a stationary process

Lemma 1.3.5: Let $0 \equiv t_{0}<t_{1}<\ldots<t_{k+1} \equiv 1$. Then

$$
\mathbb{S}\left(t_{1}\right)-\mathbb{S}\left(t_{0}\right), \ldots, \mathbb{S}\left(t_{k+1}\right)-\mathbb{S}\left(t_{k}\right)
$$


are independent random variables distributed as $N\left(0, t_{1}-t_{0}\right), \ldots, N\left(0, t_{k+1}-t_{k}\right)$ for all $k \geq 1$. Also $\mathbb{S}(0)=0$.

Proof: Follows directly from the definition of Brownian motion.

Definition 1.3.6: Let $\mathbb{U}$ be a Gaussian process. If $E[\mathbb{U}(t)]=0$ and

$$
\operatorname{Cov}\left(\mathbb{U}(t), \mathbb{U}\left(t^{\prime}\right)\right)=t \wedge t^{\prime}-t t^{\prime}
$$

for $0 \leq t, t^{\prime} \leq 1$, then $\mathbb{U}$ is Brownian bridge.

Definition 1.3.7: Let $\mathbb{X}$ be a Gaussian process. If $E[\mathbb{X}(t)]=0$ and

$$
\operatorname{Cov}\left(\mathbb{X}(t), \mathbb{X}\left(t^{\prime}\right)\right)=\exp \left(-\left|t-t^{\prime}\right|\right)
$$

for all $t, t^{\prime} \in \mathbb{R}$, then $\mathbb{X}$ is the Ornstein-Uhlenbeck process.

Definition 1.3.8: Let $\mathbb{S}$ be a Gaussian process. If $E\left[\mathbb{S}\left(t_{1}, t_{2}\right)\right]=0$ and

$$
\operatorname{Cov}\left(\mathbb{S}\left(t_{1}, t_{2}\right), \mathbb{S}\left(t_{1}^{\prime}, t_{2}^{\prime}\right)\right)=\left(t_{1} \wedge t_{1}^{\prime}\right)\left(t_{2} \wedge t_{2}^{\prime}\right)
$$

for all $t_{1}, t_{2}, t_{1}^{\prime}, t_{2}^{\prime} \in \mathbb{R}^{+}$, then $\mathbb{S}$ is the Brownian motion in 2-dimensional time.

Definition 1.3.9: Let $\mathbb{K}$ be a Gaussian process. If $E\left[\mathbb{K}\left(t_{1}, t_{2}\right)\right]=0$ and

$$
\operatorname{Cov}\left(\mathbb{K}\left(t_{1}, t_{2}\right), \mathbb{K}\left(t_{1}^{\prime}, t_{2}^{\prime}\right)\right)=\left(t_{1} \wedge t_{1}^{\prime}\right)\left(t_{2} \wedge t_{2}^{\prime}-t_{2} t_{2}^{\prime}\right)
$$

for all $t_{1}, t_{1}^{\prime} \in \mathbb{R}^{+}$and $t_{2}, t_{2}^{\prime} \in[0,1]$, then $\mathbb{K}$ is the Keifer process. Notice that this is Brownian motion in the first dimension and Brownian bridge in the second dimension.

Lemma 1.3.10: Define a random element $\mathbb{V}$ to be

$$
\mathbb{V}(t)=\mathbb{S}(t)-t \mathbb{S}(1) ; \quad 0 \leq t \leq 1
$$

Then $\mathbb{V}$ is Brownian bridge if $\mathbb{S}$ is Brownian motion. 


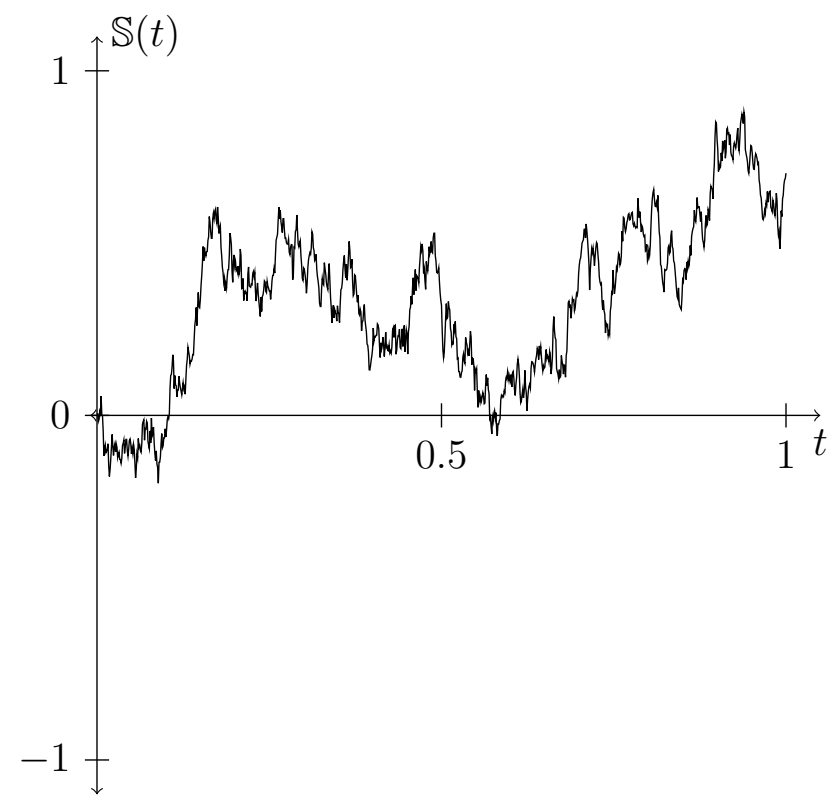

Figure 1.2: Simulation of Brownian motion path

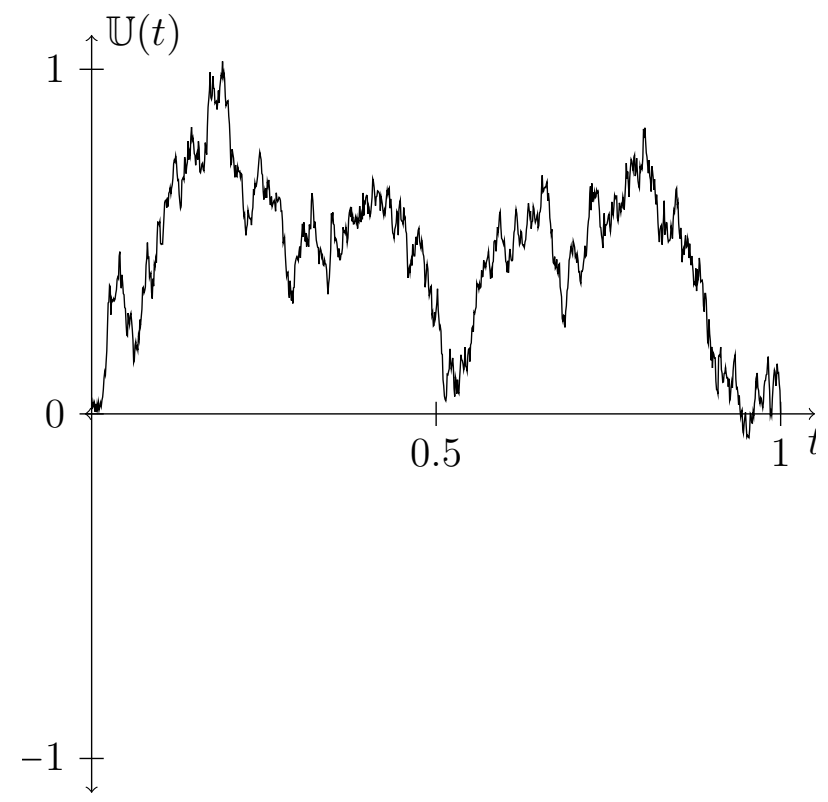

Figure 1.3: Simulation of Brownian Bridge path 


\section{Proof:}

$$
\begin{aligned}
E\left[\mathbb{V}(t) \mathbb{V}\left(t^{\prime}\right)\right] & =E\left[(\mathbb{S}(t)-t \mathbb{S}(1))\left(\mathbb{S}\left(t^{\prime}\right)-t^{\prime} \mathbb{S}(1)\right)\right] \\
& =E\left[\mathbb{S}(t) \mathbb{S}\left(t^{\prime}\right)\right]-E\left[t \mathbb{S}(1) \mathbb{S}\left(t^{\prime}\right)\right]-E\left[t^{\prime} \mathbb{S}(t) \mathbb{S}(1)\right]+E\left[t t^{\prime} \mathbb{S}(1) \mathbb{S}(1)\right] \\
& =t \wedge t^{\prime}-t t^{\prime}-t t^{\prime}+t t^{\prime} \\
& =t \wedge t^{\prime}-t t^{\prime}
\end{aligned}
$$

Therefore $\mathbb{V}$ is Brownian bridge.

Lemma 1.3.11: Let $\mathbb{U}$ be Brownian bridge on $[0,1]$. Then

$$
\mathbb{S}(t)=(1+t) \mathbb{U}\left(\frac{t}{1+t}\right) ; \quad t \geq 0
$$

is Brownian motion on $\mathbb{R}^{+}$.

\section{Proof:}

$$
\begin{aligned}
E\left[\mathbb{S}(t) \mathbb{S}\left(t^{\prime}\right)\right] & =E\left[(1+t) \mathbb{U}\left(\frac{t}{1+t}\right)\left(1+t^{\prime}\right) \mathbb{U}\left(\frac{t^{\prime}}{1+t^{\prime}}\right)\right] \\
& =(1+t)\left(1+t^{\prime}\right)\left[\min \left\{\frac{t}{1+t}, \frac{t^{\prime}}{1+t^{\prime}}\right\}-\frac{t t^{\prime}}{(1+t)\left(1+t^{\prime}\right)}\right] \\
& =\min \left\{t\left(1+t^{\prime}\right), t^{\prime}(1+t)\right\}-t t^{\prime} \\
& =t \wedge t^{\prime}
\end{aligned}
$$

Therefore $\mathbb{S}(t)$ is Brownian motion.

Lemma 1.3.12: If $\mathbb{S}$ is Brownian motion on $\mathbb{R}^{+}$and $r>0$, then

$$
\sqrt{r} \mathbb{S}\left(\frac{t}{r}\right)
$$

is Brownian motion. 


\section{Proof:}

$$
\begin{aligned}
\operatorname{Cov}\left(\sqrt{r} \mathbb{S}\left(\frac{t}{r}\right), \sqrt{r} \mathbb{S}\left(\frac{t^{\prime}}{r}\right)\right) & =E\left[r \mathbb{S}\left(\frac{t}{r}\right) \mathbb{S}\left(\frac{t^{\prime}}{r}\right)\right] \\
& =r \min \left\{\frac{t}{r}, \frac{t^{\prime}}{r}\right\}=t \wedge t^{\prime}
\end{aligned}
$$

Therefore $\sqrt{r} \mathbb{S}\left(\frac{t}{r}\right)$ is Brownian motion.

Lemma 1.3.13: If $\mathbb{S}$ is Brownian motion on $\mathbb{R}^{+}$and $r>0$, then

$$
\mathbb{S}(t+r)-\mathbb{S}(r)
$$

is Brownian motion.

\section{Proof:}

$$
\begin{aligned}
& E\left[(\mathbb{S}(t+r)-\mathbb{S}(r))\left(\mathbb{S}\left(t^{\prime}+r\right)-\mathbb{S}(r)\right)\right] \\
& =E\left[\mathbb{S}(t+r) \mathbb{S}\left(t^{\prime}+r\right)\right]-E\left[\mathbb{S}(r) \mathbb{S}\left(t^{\prime}+r\right)\right]-E[\mathbb{S}(t+r) \mathbb{S}(r)]+E[\mathbb{S}(r) \mathbb{S}(r)] \\
& =\min \left\{t+r, t^{\prime}+r\right\}-r-r+r \\
& =t \wedge t^{\prime}
\end{aligned}
$$

Therefore $\mathbb{S}(t+r)-\mathbb{S}(r)$ is Brownian motion.

Lemma 1.3.14: Let $\mathbb{S}$ be Brownian motion on $\mathbb{R}^{+}$. Then

$$
\mathbb{U}(t)=(1-t) \mathbb{S}\left(\frac{t}{1-t}\right)
$$

is Brownian bridge. 


\section{Proof:}

$$
\begin{aligned}
E\left[\mathbb{U}(t) \mathbb{U}\left(t^{\prime}\right)\right] & =(1-t)\left(1-t^{\prime}\right) E\left[\mathbb{S}\left(\frac{t}{1-t}\right) \mathbb{S}\left(\frac{t^{\prime}}{1-t^{\prime}}\right)\right] \\
& =(1-t)\left(1-t^{\prime}\right) \min \left\{\frac{t}{1-t}, \frac{t^{\prime}}{1-t^{\prime}}\right\} \\
& =t \wedge t^{\prime}-t t^{\prime}
\end{aligned}
$$

Therefore $\mathbb{U}(t)$ is Brownian bridge.

Lemma 1.3.15: Let $S: \mathbb{U}(t) \rightarrow \mathbb{S}(t)=(1+t) \mathbb{U}\left(\frac{t}{1+t}\right)$ and $T: \mathbb{S}(t) \mapsto$ $\mathbb{U}(t)=(1-t) \mathbb{S}\left(\frac{t}{1-t}\right)$. Then $S$ and $T$ are inverse transformations of each other.

Proof: Let $\mathbb{S}(t)$ be Brownian motion. Then $T(\mathbb{S}(t))=(1-t) \mathbb{S}\left(\frac{t}{1-t}\right)$ is Brownian bridge and we will define $\mathbb{U}^{\prime}(t)=T(\mathbb{S}(t))$. Then,

$$
S(T(\mathbb{S}(t)))=(1+t) \mathbb{U}^{\prime}\left(\frac{t}{1+t}\right)=(1+t)\left(1-\frac{t}{1+t}\right) \mathbb{S}\left(\frac{\frac{t}{1+t}}{1-\frac{t}{1+t}}\right)=\mathbb{S}(t)
$$

Now let $\mathbb{U}(t)$ be Brownian bridge. Then $S(\mathbb{U}(t))=(1+t) \mathbb{U}\left(\frac{t}{1+t}\right)=\mathbb{S}^{\prime}(t)$ and

$$
T(S(\mathbb{U}(t)))=(1-t) \mathbb{S}^{\prime}\left(\frac{t}{1-t}\right)=(1-t)\left(1+\frac{t}{1-t}\right) \mathbb{U}\left(\frac{\frac{t}{1-t}}{1+\frac{t}{1-t}}\right)=\mathbb{U}(t)
$$

Lemma 1.3.16: Define $\mathbb{Z}$ to be random element by

$$
\mathbb{Z}(t)=\frac{\mathbb{U}(t)+\mathbb{U}(1-t)}{\sqrt{2}} ; \quad 0 \leq t \leq 1
$$


where $\mathbb{U}$ is Brownian bridge. Then $\mathbb{Z}$ is Brownian motion on $\left[0, \frac{1}{2}\right]$. Further

$$
\mathbb{U}\left(\frac{t}{2}\right)-\mathbb{U}\left(1-\frac{t}{2}\right) ; \quad 0 \leq t \leq 1
$$

is Brownian bridge.

\section{Proof:}

$$
\begin{aligned}
E & {\left[\mathbb{Z}(t) \mathbb{Z}\left(t^{\prime}\right)\right] } \\
= & \frac{1}{2}\left(E\left[\mathbb{U}(t) \mathbb{U}\left(t^{\prime}\right)\right]+E\left[\mathbb{U}(t) \mathbb{U}\left(1-t^{\prime}\right)\right]\right. \\
& \left.+E\left[\mathbb{U}(1-t) \mathbb{U}\left(t^{\prime}\right)\right]+E\left[\mathbb{U}(1-t) \mathbb{U}\left(1-t^{\prime}\right)\right]\right) \\
= & \frac{1}{2}\left(\min \left\{t, t^{\prime}\right\}+\min \left\{t, 1-t^{\prime}\right\}+\min \left\{1-t, t^{\prime}\right\}+\min \left\{1-t, 1-t^{\prime}\right\}-1\right)
\end{aligned}
$$

At this point it is convenient to assume $t<t^{\prime}$. If $t<t^{\prime}$, then if $\mathbb{Z}$ is Brownian motion, the covariance being calculated should be $t$.

$$
\begin{aligned}
& =\frac{1}{2}\left(t+t+t^{\prime}+1-t^{\prime}-1\right) \\
& =\frac{1}{2}(2 t)=t
\end{aligned}
$$

Therefore $\mathbb{Z}$ is Brownian motion.

$$
\begin{aligned}
E & {\left[\left(\mathbb{U}\left(\frac{t}{2}\right)-\mathbb{U}\left(1-\frac{t}{2}\right)\right)\left(\mathbb{U}\left(\frac{t^{\prime}}{2}\right)-\mathbb{U}\left(1-\frac{t^{\prime}}{2}\right)\right)\right] } \\
= & \frac{1}{2} \min \left\{t, t^{\prime}\right\}-\frac{t t^{\prime}}{4}-\min \left\{\frac{t}{2}, 1-\frac{t^{\prime}}{2}\right\}+\frac{t}{2}-\frac{t t^{\prime}}{4} \\
& -\min \left\{\frac{t^{\prime}}{2}, 1-\frac{t}{2}\right\}+\frac{t^{\prime}}{2}-\frac{t t^{\prime}}{4}+\min \left\{1-\frac{t}{2}, 1-\frac{t^{\prime}}{2}\right\}-1+\frac{t}{2}+\frac{t^{\prime}}{2}-\frac{t t^{\prime}}{4}
\end{aligned}
$$


Assume $t<t^{\prime}$. This would lead to $t-t t^{\prime}$ if this process is indeed Brownian bridge

$$
\begin{aligned}
& =\frac{t}{2}-\frac{t}{2}+\frac{t}{2}-\frac{t^{\prime}}{2}+\frac{t^{\prime}}{2}+1-\frac{t^{\prime}}{2}-1+\frac{t}{2}+\frac{t^{\prime}}{2}-t t^{\prime} \\
& =t-t t^{\prime}
\end{aligned}
$$

Therefore $\mathbb{U}\left(\frac{t}{2}\right)-\mathbb{U}\left(1-\frac{t}{2}\right)$ is Brownian bridge on $\left[0, \frac{1}{2}\right]$.

Lemma 1.3.17: Let $\mathbb{U}$ and $\mathbb{V}$ be independent Brownian bridges. Then for $0 \leq a \leq 1$

$$
\sqrt{1-a} \mathbb{U}+\sqrt{a} \mathbb{V}
$$

is also Brownian bridge.

Proof: First, note that because $\mathbb{U}$ and $\mathbb{V}$ are independent, $E\left[\mathbb{U}(t) \mathbb{V}\left(t^{\prime}\right)\right]=0$ for all $t$ and $t^{\prime}$. Therefore the cross terms can be ignored in the expansion below.

$$
\begin{aligned}
& E\left[(\sqrt{1-a} \mathbb{U}(t)+\sqrt{a} \mathbb{V}(t))\left(\sqrt{1-a} \mathbb{U}\left(t^{\prime}\right)+\sqrt{a} \mathbb{V}\left(t^{\prime}\right)\right)\right] \\
& =(1-a)\left(\mathbb{U}(t) \mathbb{U}\left(t^{\prime}\right)\right)+a\left(\mathbb{V}(t) \mathbb{V}\left(t^{\prime}\right)\right) \\
& =(1-a)\left(t \wedge t^{\prime}-t t^{\prime}\right)+a\left(t \wedge t^{\prime}-t t^{\prime}\right)
\end{aligned}
$$

Therefore $\sqrt{1-a} \mathbb{U}-\sqrt{a} \mathbb{V}$ is Brownian bridge.

Lemma 1.3.18: Let $\mathbb{U}$ be Brownian bridge and $Z$ be a $N(0,1)$ random variable independent of $\mathbb{U}$. Then $\mathbb{U}+t Z$ is Brownian motion on $[0,1]$.

Proof: First, note that because $\mathbb{U}$ and $Z$ are independent, $E[\mathbb{U} Z]=0$. Therefore the cross terms can be ignored in the expansion below.

$$
\begin{aligned}
E\left[(\mathbb{U}(t)+t Z)\left(\mathbb{U}\left(t^{\prime}\right)+t^{\prime} Z\right)\right] & =E\left[\mathbb{U}(t) \mathbb{U}\left(t^{\prime}\right)\right]+t t^{\prime} E\left[Z^{2}\right] \\
& =t \wedge t^{\prime}-t t^{\prime}+t t^{\prime}=t \wedge t^{\prime}
\end{aligned}
$$

Therefore $\mathbb{U}+t Z$ is Brownian motion. 
Lemma 1.3.19: $\mathbb{S}(1)$ and $\mathbb{U}(t)$ are independent.

Proof: $E[\mathbb{S}(1) \mathbb{U}(t)]=E[\mathbb{S}(1) \mathbb{S}(t)]-t E[\mathbb{S}(1) \mathbb{S}(1)]=t-t=0$

Lemma 1.3.20: Let $\mathbb{S}$ be Brownian motion on $[0, \infty)$. Then the time reversal process

$$
t \mathbb{S}\left(\frac{1}{t}\right)
$$

for $t \geq 0$ is also Brownian motion.

\section{Proof:}

$$
\begin{aligned}
E\left[t \mathbb{S}\left(\frac{1}{t}\right) t^{\prime} \mathbb{S}\left(\frac{1}{t^{\prime}}\right)\right] & =t t^{\prime} \min \left\{\frac{1}{t}, \frac{1}{t^{\prime}}\right\} \\
& =t^{\prime} \wedge t
\end{aligned}
$$

Therefore $t \mathbb{S}\left(\frac{1}{t}\right)$ is Brownian motion.

Lemma 1.3.21: Let $\mathbb{S}$ be Brownian motion on $\mathbb{R}^{+}$. Then

$$
\mathbb{X}(t)=e^{-t} \mathbb{S}\left(e^{2 t}\right)
$$

is a Ornstein-Uhlenbeck process.

\section{Proof:}

$$
\begin{aligned}
\operatorname{Cov}\left(e^{-t} \mathbb{S}\left(e^{2 t}\right), e^{-t^{\prime}} \mathbb{S}\left(e^{2 t^{\prime}}\right)\right) & =e^{-t-t^{\prime}} E\left[\mathbb{S}\left(e^{2 t}\right) \mathbb{S}\left(e^{2 t^{\prime}}\right)\right] \\
& =e^{-t-t^{\prime}} \min \left\{e^{2 t}, e^{2 t^{\prime}}\right\} \\
& =e^{-\left|t-t^{\prime}\right|}
\end{aligned}
$$

Therefore $\mathbb{X}(t)$ is an Ornstein-Uhlenbeck process. 
Leмma 1.3.22: Let $\mathbb{S}$ be a Ornstein-Uhlenbeck process on $\mathbb{R}$. Then

$$
\mathbb{U}(t)= \begin{cases}\sqrt{t(1-t) \mathbb{X}}\left(\frac{1}{2} \log \left(\frac{t}{1-t}\right)\right) & 0 \leq t \leq 1 \\ 0 & t=0 \text { or } t=1\end{cases}
$$

is Brownian bridge on $[0,1]$.

\section{Proof:}

$$
E\left[\mathbb{U}(t) \mathbb{U}\left(t^{\prime}\right)\right]=E\left[\sqrt{t t^{\prime}(1-t)\left(1-t^{\prime}\right) \mathbb{X}}\left(\frac{1}{2} \log \left(\frac{t}{1-t}\right)\right) \mathbb{X}\left(\frac{1}{2} \log \left(\frac{t^{\prime}}{1-t^{\prime}}\right)\right)\right]
$$

Assuming $t<t^{\prime}$

$$
\begin{aligned}
& =\sqrt{t t^{\prime}(1-t)\left(1-t^{\prime}\right)} \exp \left(-\log \left(\left(\frac{t\left(1-t^{\prime}\right)}{t^{\prime}(1-t)}\right)^{\frac{1}{2}}\right)\right) \\
& =\sqrt{t t^{\prime}(1-t)\left(1-t^{\prime}\right)} \sqrt{\frac{t\left(1-t^{\prime}\right)}{t^{\prime}(1-t)}}=t-t t^{\prime}
\end{aligned}
$$

Therefore it is Brownian bridge.

Lemma 1.3.23: Let $\mathbb{U}$ be a Brownian bridge. Define

$$
\mathbb{B}(t)=\frac{[\mathbb{U}(a t)-t \mathbb{U}(a)]}{\sqrt{a}}
$$

for fixed $0<a \leq 1$. Then $\mathbb{B}$ is Brownian bridge on $[0,1]$ and is independent of $\{\mathbb{U}(t): a \leq t \leq 1\}$. 


\section{Proof:}

$$
\begin{aligned}
& E\left[\mathbb{B}(t) \mathbb{B}\left(t^{\prime}\right)\right] \\
& =\frac{1}{a}\left(E\left[\mathbb{U}(a t) \mathbb{U}\left(a t^{\prime}\right)-\mathbb{U}(a t) t^{\prime} \mathbb{U}(a)-\mathbb{U}\left(a t^{\prime}\right) t \mathbb{U}(a)+t t^{\prime} \mathbb{U}(a) \mathbb{U}(a)\right]\right) \\
& =\frac{1}{a}\left(a t \wedge a t^{\prime}-a^{2} t t^{\prime}-t^{\prime}\left(a t-a^{2} t\right)-t\left(a t^{\prime}-a t^{\prime 2}\right)+t t^{\prime} a(1-a)\right) \\
& =t \wedge t^{\prime}-t t^{\prime}
\end{aligned}
$$

To show independence consider the covariance between $\mathbb{B}$ and $\mathbb{U}$ $\left(0 \leq t \leq a \leq t^{\prime} \leq 1\right)$.

$$
\begin{aligned}
E\left[\mathbb{B}(t) \mathbb{U}\left(t^{\prime}\right)\right] & =\frac{1}{\sqrt{a}}\left(E\left[\mathbb{U}(a t) \mathbb{U}\left(t^{\prime}\right)\right]-t E\left[\mathbb{U}(a) \mathbb{U}\left(t^{\prime}\right)\right]\right) \\
& =\frac{1}{\sqrt{a}}\left(a t-a t t^{\prime}-t\left(a-a t t^{\prime}\right)\right) \\
& =0
\end{aligned}
$$

As they are uncorrelated and Gaussian, they are independent.

Lemma 1.3.24: Let $\mathbb{S}$ be Brownian motion and $\mathbb{U}$ be Brownian bridge. Then

1. $\mathbb{S}(t)-\int_{0}^{t} \frac{\mathbb{S}(r)}{r} d r ; \quad 0 \leq t \leq 1$

2. $\mathbb{U}(t)-\int_{0}^{t} \frac{\mathbb{U}(r)}{r} d r ; \quad 0 \leq t \leq 1$

3. $\mathbb{U}(t)+\int_{0}^{t} \frac{\mathbb{U}(r)}{1-r} d r ; \quad 0 \leq t \leq 1$

are all Brownian motion.

\section{Proof:}


1. Assuming $t<t^{\prime}$

$$
\begin{aligned}
E & {\left[\left(\mathbb{S}(t)-\int_{0}^{t} \frac{\mathbb{S}(r)}{r} d r\right)\left(\mathbb{S}\left(t^{\prime}\right)-\int_{0}^{t^{\prime}} \frac{\mathbb{S}(r)}{r} d r\right)\right] } \\
= & E\left[\mathbb{S}(t) \mathbb{S}\left(t^{\prime}\right)\right]-E\left[\mathbb{S}(t) \int_{0}^{t^{\prime}} \frac{\mathbb{S}(r)}{r} d r\right]-E\left[\mathbb{S}\left(t^{\prime}\right) \int_{0}^{t} \frac{\mathbb{S}(r)}{r} d r\right] \\
& +E\left[\int_{0}^{t} \frac{\mathbb{S}(r)}{r} d r \int_{0}^{t^{\prime}} \frac{\mathbb{S}(r)}{r} d r\right] \\
= & t-\int_{0}^{t} \frac{E[\mathbb{S}(t) \mathbb{S}(r)]}{r} d r-\int_{t}^{t^{\prime}} \frac{E[\mathbb{S}(t) \mathbb{S}(r)]}{r} d r-\int_{0}^{t} \frac{E\left[\mathbb{S}\left(t^{\prime}\right) \mathbb{S}(r)\right]}{r} d r \\
& +\int_{0}^{t} \int_{0}^{t} \frac{E\left[\mathbb{S}(r) \mathbb{S}\left(r^{\prime}\right)\right]}{r r^{\prime}} d r d r^{\prime}+\int_{t}^{t^{\prime}} \int_{0}^{t} \frac{E\left[\mathbb{S}(r) \mathbb{S}\left(r^{\prime}\right)\right]}{r r^{\prime}} d r d r^{\prime} \\
= & t-\int_{0}^{t} d r-t \int_{t}^{t^{\prime}} \frac{d r}{r}-\int_{0}^{t} d r+2 \int_{0}^{t} \int_{0}^{r^{\prime}} \frac{1}{r^{\prime}} d r d r^{\prime}+\int_{t}^{t^{\prime}} \int_{0}^{t} \frac{1}{r^{\prime}} d r d r^{\prime} \\
= & t-t-t \int_{t}^{t^{\prime}} \frac{d r}{r}-t+2 t+t \int_{t}^{t^{\prime}} \frac{1}{r^{\prime}} d r^{\prime} \\
= & t
\end{aligned}
$$

Therefore $\mathbb{S}(t)-\int_{0}^{t} \frac{\mathbb{S}(r)}{r} d r ; \quad 0 \leq t \leq 1$ is Brownian motion.

2.

$$
\begin{aligned}
\mathbb{U}(t)-\int_{0}^{t} \frac{\mathbb{U}(r)}{r} d r & =\mathbb{S}(t)-t \mathbb{S}(1)-\int_{0}^{t} \frac{\mathbb{S}(r)}{r} d r-\mathbb{S}(1) \int_{0}^{t} d r \\
& =\mathbb{S}(t)-\int_{0}^{t} \frac{\mathbb{S}(r)}{r} d r
\end{aligned}
$$

Therefore using 1 , we see that $\mathbb{U}(t)-\int_{0}^{t} \frac{\mathbb{U}(r)}{r} d r ; \quad 0 \leq t \leq 1$ is Brownian motion.

3. Assume $t<t^{\prime}$

$$
\begin{aligned}
& E\left[\left(\mathbb{U}(t)+\int_{0}^{t} \frac{\mathbb{U}(r)}{1-r} d r\right)\left(\mathbb{U}\left(t^{\prime}\right)+\int_{0}^{t^{\prime}} \frac{\mathbb{U}(r)}{1-r} d r\right)\right] \\
& =E\left[\mathbb{U}(t) \mathbb{U}\left(t^{\prime}\right)\right]+\int_{0}^{t^{\prime}} \frac{E[\mathbb{U}(t) \mathbb{U}(r)]}{1-r} d r+\int_{0}^{t} \frac{E\left[\mathbb{U}\left(t^{\prime}\right) \mathbb{U}(r)\right]}{1-r} d r \\
& \quad+\int_{0}^{t^{\prime}} \int_{0}^{t} \frac{E\left[\mathbb{U}(r) \mathbb{U}\left(r^{\prime}\right)\right]}{(1-r)\left(1-r^{\prime}\right)} d r d r^{\prime}
\end{aligned}
$$


At this point it is convenient to consider the integrals seperately.

$$
\begin{aligned}
& \int_{0}^{t^{\prime}} \frac{E[\mathbb{U}(t) \mathbb{U}(r)]}{1-r} d r \\
& =\int_{0}^{t} \frac{E[\mathbb{U}(t) \mathbb{U}(r)]}{1-r} d r+\int_{t}^{t^{\prime}} \frac{E[\mathbb{U}(t) \mathbb{U}(r)]}{1-r} d r \\
& =(1-t) \int_{0}^{t} \frac{r}{1-r} d r+t\left(t^{\prime}-t\right) \\
& =-t+\int_{0}^{t} \frac{1}{1-r} d r-t \int_{0}^{t} \frac{r}{1-r} d r+t\left(t^{\prime}-t\right) \\
& \int_{0}^{t} \frac{E\left[\mathbb{U}\left(t^{\prime}\right) \mathbb{U}(r)\right]}{1-r} d r \\
& =\left(1-t^{\prime}\right) \int_{0}^{t} \frac{r}{1-r} d r \\
& =-t+\int_{0}^{t} \frac{1}{1-r} d r-t^{\prime} \int_{0}^{t} \frac{r}{1-r} d r \\
& \int_{0}^{t^{\prime}} \int_{0}^{t} \frac{E\left[\mathbb{U}(r) \mathbb{U}\left(r^{\prime}\right)\right]}{(1-r)\left(1-r^{\prime}\right)} d r d r^{\prime} \\
& =2 \int_{0}^{t} \int_{r^{\prime}}^{t} \frac{r^{\prime}}{\left(1-r^{\prime}\right)} d r d r^{\prime}+\int_{0}^{t} \int_{t}^{t^{\prime}} \frac{r^{\prime}}{\left(1-r^{\prime}\right)} d r d r^{\prime} \\
& =2 t \int_{0}^{t} \frac{r}{(1-r)} d r-2 \int_{0}^{t} \frac{r^{2}}{(1-r)} d r+\left(t^{\prime}-t\right) \int_{0}^{t} \frac{r}{(1-r)} d r \\
& =2 t \int_{0}^{t} \frac{r}{(1-r)} d r-2 \int_{0}^{t} \frac{1}{(1-r)} d r+\left(t^{2}+2 t\right)+\left(t^{\prime}-t\right) \int_{0}^{t} \frac{r}{(1-r)} d r
\end{aligned}
$$

Putting all this together gives the required result, that $\mathbb{U}(t)+\int_{0}^{t} \frac{\mathbb{U}(r)}{1-r} d r ; \quad 0 \leq t \leq 1$ is Brownian motion.

Lemma 1.3.25: The sequence of functions $\left\{\frac{\sqrt{2} \sin (k \pi t)}{k \pi}\right\}_{k=0}^{\infty}=\left\{f_{k}(t)\right\}_{k=0}^{\infty}$ are orthogonal on $0 \leq t \leq 1$.

Proof: Let $k \neq j$, then

$$
\int_{0}^{1} f_{k}(t) f_{j}(t) d t=\frac{2}{k j \pi^{2}} \int_{0}^{1} \sin (k \pi t) \sin (j \pi t) d t
$$


Using integration by parts

$$
=\frac{2}{k j \pi^{2}}\left[-\left.\frac{\sin (k \pi t) \cos (j \pi t)}{j \pi}\right|_{0} ^{1}+\frac{k}{j} \int_{0}^{1} \cos (k \pi t) \cos (j \pi t) d t\right]
$$

Using integration by parts again and evaluating the first expression, letting $I=\int_{0}^{1} f_{k}(t) f_{j}(t) d t$

$$
\begin{aligned}
I & =\frac{2}{k j \pi^{2}} \frac{k}{j}\left[\left.\frac{\cos (k \pi t) \sin (j \pi t)}{j \pi}\right|_{0} ^{1}+\frac{k}{j} \int_{0}^{1} \sin (k \pi t) \sin (j \pi t) d t\right] \\
& =\frac{k^{2}}{j^{2}} I
\end{aligned}
$$

As $k \neq j, I=0$.

Theorem 1.3.26: Let $Z_{0}, Z_{1}, \ldots$ be i.i.d. $N(0,1)$ random variables. Define

$$
\mathbb{U}(t) \equiv \sum_{k=1}^{\infty} Z_{k} \frac{\sqrt{2} \sin (k \pi t)}{k \pi} ; \quad 0 \leq t \leq 1
$$

and

$$
\mathbb{S}(t) \equiv Z_{0} t+\sum_{k=1}^{\infty} Z_{k} \frac{\sqrt{2} \sin (k \pi t)}{k \pi} ; \quad 0 \leq t \leq 1
$$

then $\mathbb{U}$ is Brownian bridge and $\mathbb{S}$ is Brownian motion on $[0,1]$. This establishes the existence of Brownian motion and Brownian bridge in 1-dimension.

\section{Proof:}

$$
\begin{aligned}
& \operatorname{Cov}\left(\mathbb{U}(t), \mathbb{U}\left(t^{\prime}\right)\right) \\
& =E\left[\left(\sum_{k=1}^{\infty} Z_{k} \frac{\sqrt{2} \sin (k \pi t)}{k \pi}\right)\left(\sum_{j=1}^{\infty} Z_{j} \frac{\sqrt{2} \sin (j \pi t)}{j \pi}\right)\right]
\end{aligned}
$$


As $Z_{i} Z_{j}$ are independent for $i \neq j$ the expected value of these is 0 , so

$$
\begin{aligned}
& =\sum_{k=1}^{\infty} E\left[Z_{k}^{2} \frac{2 \sin (k \pi t) \sin \left(k \pi t^{\prime}\right)}{k^{2} \pi^{2}}\right] \\
& =\sum_{k=1}^{\infty} \frac{2 \sin (k \pi t) \sin \left(k \pi t^{\prime}\right)}{k^{2} \pi^{2}}
\end{aligned}
$$

which is seen to be the bivariate Fourier series of $t \wedge t^{\prime}-t t^{\prime}$. To show $\mathbb{S}$ is Brownian motion use LEMMA 1.3.18 as

$$
\mathbb{S}(t) \equiv Z_{0} t+\sum_{k=1}^{\infty} Z_{k} \frac{\sqrt{2} \sin (k \pi t)}{k \pi}=Z_{0} t+\mathbb{U}(t)
$$

Lemma 1.3.27: Let $\Pi: \mathbb{S}(t) \mapsto \mathbb{S}(t)-t \mathbb{S}(1)$ and $\Upsilon: \mathbb{U}(t) \mapsto(1+t) \mathbb{U}\left(\frac{t}{1+t}\right)$. Begin with $\mathbb{S}(t)=\mathbb{S}^{0}(t)$ Brownian motion and define inductively $\mathbb{U}^{i}(t)=$ $\Pi\left(\mathbb{S}^{i}(t)\right)$ for $i=0,1, \ldots$ and $\mathbb{S}^{i}=\Upsilon\left(\mathbb{U}^{i-1}(t)\right)$ for $i=1,2, \ldots$ Then $\mathbb{U}^{i}(t)$ are Brownian bridges and $\mathbb{S}^{i}(t)$ are Brownian motions, with

$$
\mathbb{S}^{n}(t)=(1+n t) \mathbb{S}\left(\frac{t}{1+n t}\right)-n t \mathbb{S}\left(\frac{1}{n}\right)
$$

Proof: Assuming that $\mathbb{S}^{n}(t)=(1+n t) \mathbb{S}\left(\frac{t}{1+n t}\right)-n t \mathbb{S}\left(\frac{1}{n}\right)$ then

$$
\begin{aligned}
\mathbb{U}^{n}(t) & =\Pi\left(\mathbb{S}^{n}(t)\right) \\
& =(1+n t) \mathbb{S}\left(\frac{t}{1+n t}\right)-n t \mathbb{S}\left(\frac{1}{n}\right)-(n+1) t \mathbb{S}\left(\frac{1}{n+1}\right)+n t \mathbb{S}\left(\frac{1}{n}\right) \\
& =(1+n t) \mathbb{S}\left(\frac{t}{1+n t}\right)-(n+1) t \mathbb{S}\left(\frac{1}{n+1}\right)
\end{aligned}
$$




$$
\begin{aligned}
\mathbb{S}^{n+1}(t) & =\Upsilon\left(\mathbb{U}^{n}(t)\right) \\
& =(1+t)\left(1+\frac{n t}{1+t}\right) \mathbb{S}\left(\frac{\frac{t}{1+t}}{1+\frac{n t}{1+t}}\right)-(1+t)(n+1) \frac{t}{1+t} \mathbb{S}\left(\frac{1}{n+1}\right) \\
& =\mathbb{S}^{n+1}(t)=(1+(n+1) t) \mathbb{S}\left(\frac{t}{1+(n+1) t}\right)-(n+1) t \mathbb{S}\left(\frac{1}{n+1}\right)
\end{aligned}
$$

Using LEMmA 1.3.11 and LEMmA 1.3.10 completes the proof by induction.

\subsubsection{REFLECTION PRINCIPLES}

The following subsection follows SHORACK \& WELLner (1986).

Theorem 1.3.28: Let $\mathbb{S}$ be Brownian motion and $T_{x}$ be the first moment the Brownian motion crosses level $x$. Then for $b>0$

$$
\mathbb{P}\left\{T_{b}<t\right\}=\int_{0}^{t} \frac{b}{\sqrt{2 \pi s^{3}}} \exp \left(-\frac{b^{2}}{2 s}\right) d s
$$

Proof: This proof follows the proof given in FELLER (1971a).

$$
\mathbb{P}\left\{T_{b}<t\right\}=\mathbb{P}\left\{T_{b}<t, \mathbb{S}(t)>b\right\}+\mathbb{P}\left\{T_{b}<t, \mathbb{S}(t) \leq b\right\}
$$


As $\mathbb{S}(t)$ is continuous, $\mathbb{P}\left\{T_{b}<t, \mathbb{S}(t)>b\right\}=\mathbb{P}\{\mathbb{S}(t)>b\}$. Consider the second term. At $\tau$, using the strong Markov property we have a new Brownian motion $(\mathbb{S}(t)-\mathbb{S}(\tau))$ with respect to the starting point $(\tau, b)$. Therefore the probability of $\mathbb{S}(t) \leq b$ is $\frac{1}{2}$, the same as the probability of Brownian motion starting at 0 being less than 0 at any given $t \geq 0$.

$$
\begin{aligned}
& =\mathbb{P}\{\mathbb{S}(t)>b\}+\frac{1}{2} \mathbb{P}\left\{T_{b}<t\right\} \\
\Rightarrow \mathbb{P}\left\{T_{b}<t\right\} & =2 \mathbb{P}\{\mathbb{S}(t)>b\} \\
& =2\left(1-\Phi\left(\frac{b}{\sqrt{t}}\right)\right) \\
& =2 \int_{0}^{t} \frac{d}{d s}\left(1-\Phi\left(\frac{b}{\sqrt{s}}\right)\right) d s \\
& =2 \int_{0}^{t} \frac{b}{2 \sqrt{2 \pi s^{3}}} \exp \left(-\frac{b^{2}}{2 s}\right) d s=\int_{0}^{t} \frac{b}{\sqrt{2 \pi s^{3}}} \exp \left(-\frac{b^{2}}{2 s}\right) d s
\end{aligned}
$$

Theorem 1.3.29: Let $\mathbb{S}$ be Brownian motion. Then for $b>0$

$$
\begin{aligned}
\mathbb{P}\left\{\sup _{0 \leq s \leq t}|\mathbb{S}(s)| \geq b\right\} & =4 \sum_{k=0}^{\infty} \mathbb{P}\{(4 k-3) b<N(0, t)<(4 k-1) b\} \\
& =1-\sum_{k=-\infty}^{\infty}(-1)^{k} \mathbb{P}\{(2 k-1) b<N(0, t)<(2 k+1) b\}
\end{aligned}
$$

Proof: To prove the main result it is first required to define

$$
\begin{aligned}
& \left\{A_{+}\right\} \equiv\{\mathbb{S} \text { crosses the level } b \text { in }[0, t]\} \\
& \left\{A_{-}\right\} \equiv\{\mathbb{S} \text { crosses the level }-b \text { in }[0, t]\}
\end{aligned}
$$

This allows us to inductively define

$$
\begin{aligned}
& \left\{A_{+-}\right\} \equiv\{\mathbb{S} \text { crosses the level } b \text { then crosses level }-b \text { in }[0, t]\} \\
& \left\{A_{-+}\right\} \equiv\{\mathbb{S} \text { crosses the level }-b \text { then crosses level }+b \text { in }[0, t]\}
\end{aligned}
$$


and similarly for $A_{+-+}, A_{-+-}, A_{+-+-}, \ldots$. The idea is to expand out the event $\left\{\sup _{0 \leq s \leq t}|\mathbb{S}(s)| \geq b\right\}$ in terms of the events defined above. Clearly

$$
\mathbb{P}\left\{\sup _{0 \leq s \leq t}|\mathbb{S}(s)| \geq b\right\}<\mathbb{P}\left\{A_{+}\right\}+\mathbb{P}\left\{A_{-}\right\}
$$

as the event where $\mathbb{S}$ crosses both $b$ and $-b$ is counted twice. So then

$$
\mathbb{P}\left\{\sup _{0 \leq s \leq t}|\mathbb{S}(s)| \geq b\right\}>\mathbb{P}\left\{A_{+}\right\}-\mathbb{P}\left\{A_{+-}\right\}+\mathbb{P}\left\{A_{-}\right\}-\mathbb{P}\left\{A_{-+}\right\}
$$

This leads us to conclude that

$$
\begin{aligned}
\mathbb{P}\left\{\sup _{0 \leq s \leq t}|\mathbb{S}(s)| \geq b\right\} & =\mathbb{P}\left\{A_{+}\right\}-\mathbb{P}\left\{A_{+-}\right\}+\mathbb{P}\left\{A_{+-+}\right\}-\ldots \\
& +\mathbb{P}\left\{A_{-}\right\}-\mathbb{P}\left\{A_{-+}\right\}+\mathbb{P}\left\{A_{-+-}\right\}-\ldots
\end{aligned}
$$

By symmetry

$$
=2\left(\mathbb{P}\left\{A_{+}\right\}-\mathbb{P}\left\{A_{+-}\right\}+\mathbb{P}\left\{A_{+-+}\right\}-\ldots\right)
$$

Using the idea in Figure 1.4 and Theorem 1.3.28

$$
=2 \sum_{k=1}^{\infty}(-1)^{k+1} 2 \mathbb{P}\{N(0, t)>(2 k-1) b\}
$$

By considering the area remaining under the graph of the normal density after each $k$ in FiguRE 1.5

$$
\begin{aligned}
& =4 \sum_{k=1}^{\infty} \mathbb{P}\{(4 k-3) b<N(0, t)<(4 k-1) b\} \\
& =1-\sum_{k=-\infty}^{\infty}(-1)^{k} \mathbb{P}\{(2 k-1) b<N(0, t)<(2 k+1) b\}
\end{aligned}
$$




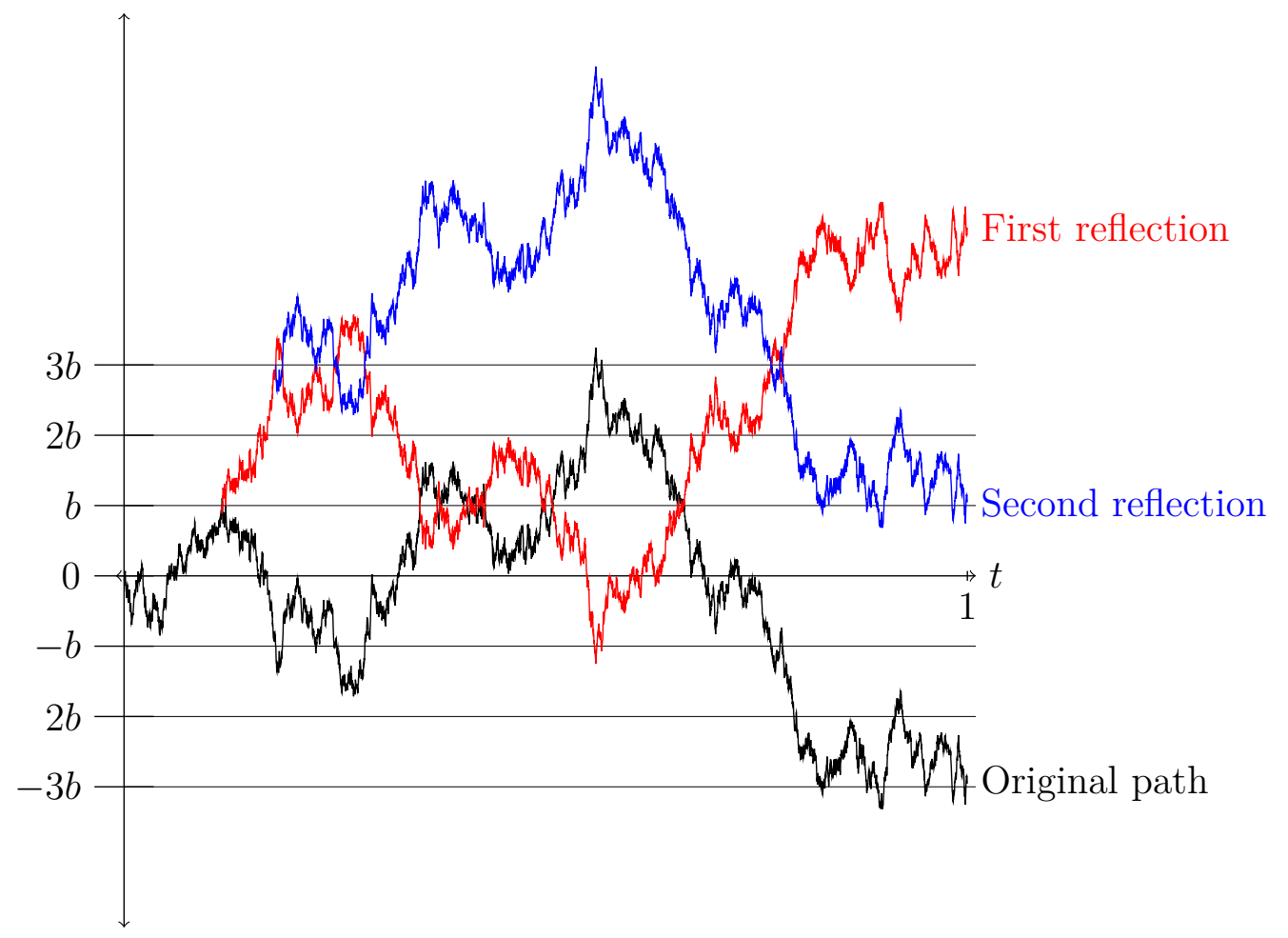

Figure 1.4: The red line is the reflection of the Brownian motion in the line $y=b$ after the Brownian motion hits $b$. The blue line is the reflection of the red line in the line $y=3 b$ after the Brownian motion hits $y=-b$. This justifies why $\mathbb{S}(t) \geq b$ and $\mathbb{S}(t) \leq-b$ is the same as $\mathbb{S}(t) \geq 3 b$. An extension of this idea to when the Brownian motion repeatedly crosses the levels $b$ and $-b$ is used in THEOREM 1.3.29 


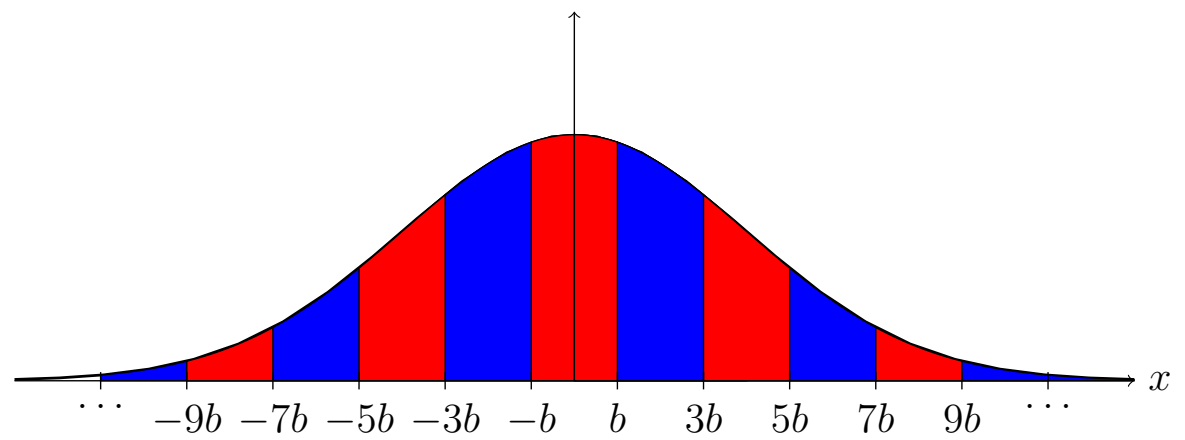

Figure 1.5: $\sum_{k=1}^{\infty} \mathbb{P}\{(4 k-3) b<N(0,1)<(4 k-1) b\}$ is the blue area on the positive half axis. By symmetry this is equal to the blue area in the negative half axis and red area $=1-$ blue area. Using symmetry and simplifying we get $2 \sum_{k=1}^{\infty}(-1)^{k+1} 2 \mathbb{P}\{N(0,1)>(2 k-1) b\}=1-$ red area + blue area.

Theorem 1.3.30: Let $\mathbb{S}(t)$ be Brownian motion. For $a \geq 0$ and $b>0$

$$
\mathbb{P}\left\{\sup _{t \geq 0} \frac{\mathbb{S}(t)}{a t+b} \geq 1\right\}=\exp (-2 a b)
$$

Proof: We first note some properties. Let $\phi(a, b)=\mathbb{P}\left\{\sup _{t \geq 0} \mathbb{S}(t) \geq a t+b\right\}$. Then $\phi(a, b) \geq \mathbb{P}\{\mathbb{S}(t) \geq a+b\}>0$ for all $a \geq 0$ and $b>0, \phi(a, b)$ decreases in $b$ and $\phi\left(a, b_{1}+b_{2}\right)=\phi\left(a, b_{1}\right) \phi\left(a, b_{2}\right)$, which is shown in Figure 1.6. The only solution of the functional equation $\phi\left(a, b_{1}+b_{2}\right)=\phi\left(a, b_{1}\right) \phi\left(a, b_{2}\right)$ in $b$ is

$$
\begin{gathered}
\phi(a, b)=\exp (-\psi(a) b) \\
\exp (-\psi(a) b)=\phi(a, b) \\
=E_{\tau}[\phi(a, a \tau)]
\end{gathered}
$$




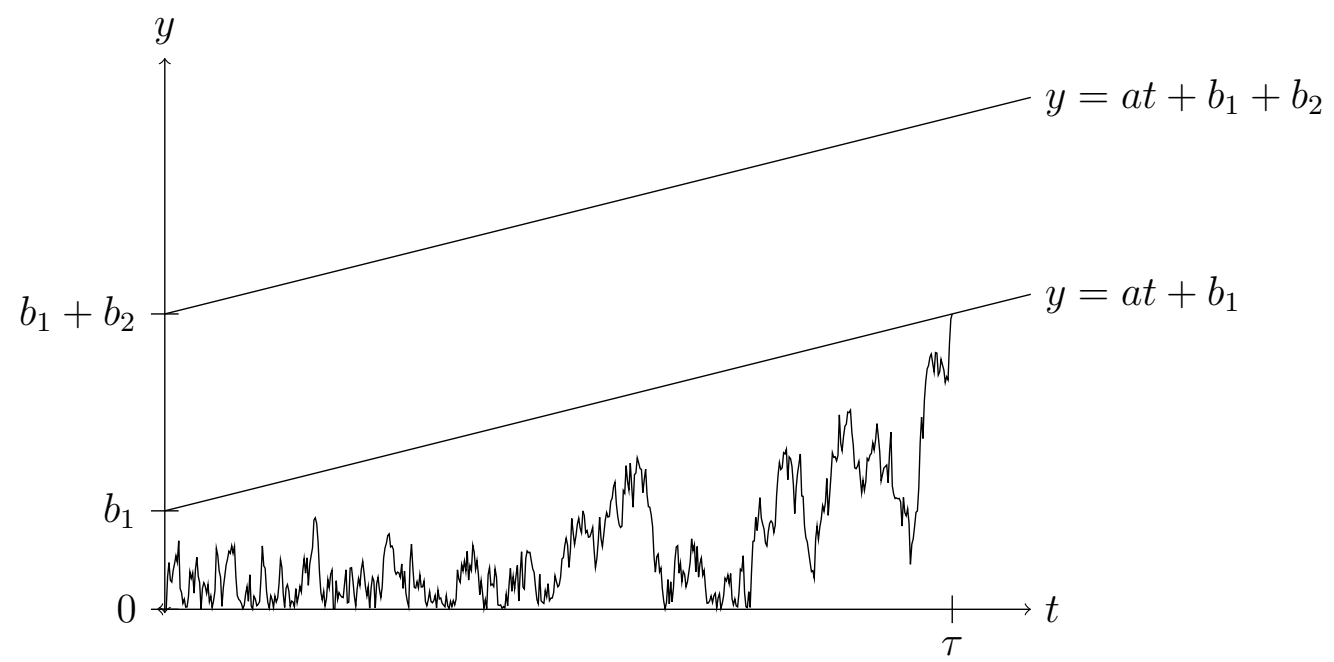

FiguRE 1.6: At the instant $\tau$ when $\mathbb{S}(\tau)=a \tau+b_{1}$, we have the same problem again, but with $\phi\left(a, b_{2}\right)$ relative to the point $(\tau, \mathbb{S}(\tau))$

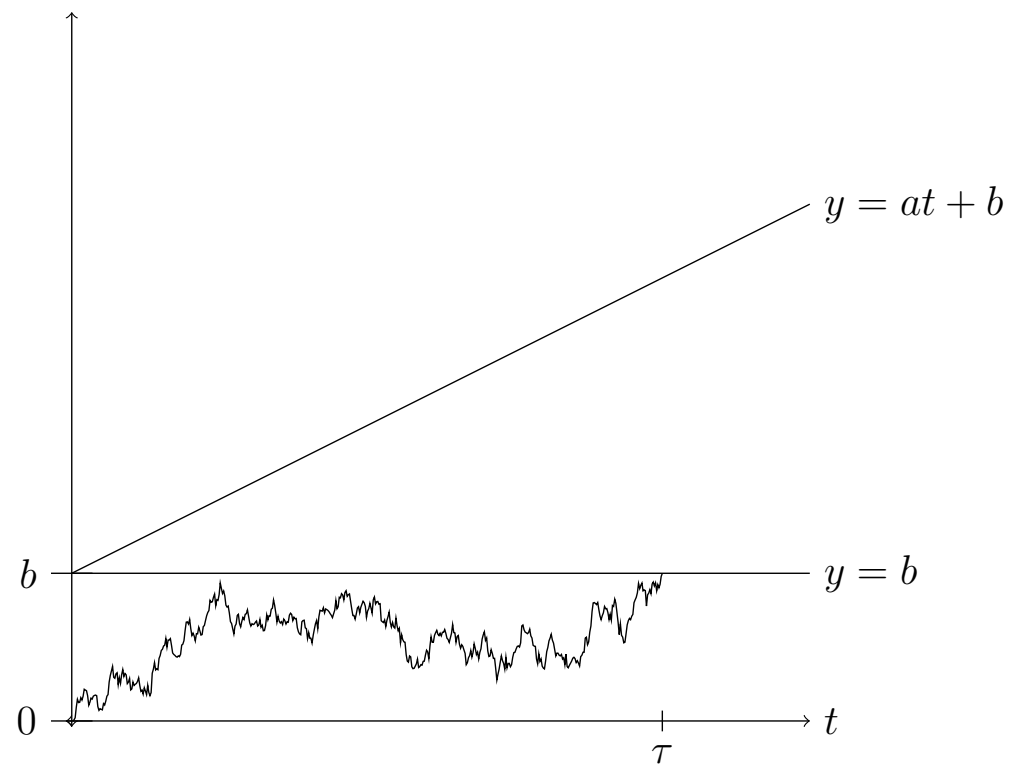

FiguRE 1.7: Relative to $(\tau, b)$, the sloping line has equation $y=a t+a \tau$, where $\tau$ is the first instant $\mathbb{S}(t)=b$. 
Using THEOREM 1.3.28

$$
=\int_{0}^{\infty} \exp (-\psi(a) a s) \frac{b}{\sqrt{2 \pi s^{3}}} \exp \left(-\frac{b^{2}}{2 s}\right) d s
$$

Let $y^{2}=\frac{b^{2}}{2 s}$

$$
=\frac{2}{\sqrt{\pi}} \int_{0}^{\infty} \exp \left(-\left(y^{2}+\frac{c}{y^{2}}\right)\right) d y
$$

where $c=\frac{b^{2} a \psi(a)}{2}$.

$$
=\exp (-b \sqrt{2 a \psi(a)})
$$

Therefore $\psi(a)=2 a$ and

$$
\mathbb{P}\left\{\sup _{t \geq 0} \frac{\mathbb{S}(t)}{a t+b} \geq 1\right\}=\exp (-2 a b)
$$

Theorem 1.3.31: Let $\mathbb{U}$ be Brownian bridge. For all $b>0$

$$
\mathbb{P}\left\{\sup _{0 \leq t \leq 1} \mathbb{U}(t)>b\right\}=\exp \left(-2 b^{2}\right)
$$

Proof: Using Lemma 1.3.14

$$
\begin{aligned}
\mathbb{P}\left\{\sup _{0 \leq t \leq 1} \mathbb{U}(t)>b\right\} & =\mathbb{P}\left\{\sup _{0 \leq t \leq 1}(1-t) \mathbb{S}\left(\frac{t}{1-t}\right)>b\right\} \\
& =\mathbb{P}\left\{\sup _{r>0} \frac{\mathbb{S}(r)}{1+r}>b\right\}
\end{aligned}
$$


Using TheOREM 1.3.30

$$
=\exp \left(-2 b^{2}\right)
$$

Theorem 1.3.32: Let $\mathbb{S}(t)$ be Brownian motion. Then for $a \geq 0$ and $b>0$

$$
\mathbb{P}\left\{\sup _{t \geq 0} \frac{|\mathbb{S}(s)|}{a t+b} \geq 1\right\}=2 \sum_{k=1}^{\infty}(-1)^{k+1} \exp \left(-2 k^{2} a b\right)
$$

Proof: Following Doob (1949), consider the more general problem of Brownian motion intersecting a sequence of lines. Let $\left\{\left[u_{n}, v_{n}\right]\right\}_{n=1}^{\infty}$ be a sequence of lines in the form $u_{n} t+v_{n}$ for $u_{n} \geq 0, v_{n} \geq 0$. Let $t_{1}$ be the first instant that $\mathbb{S}(t)$ intersects $\left[u_{1}, v_{1}\right]$, if such a point exists; if $t_{1}$ is defined, then define $t_{2}$ to be the first instant $t>t_{1}$ that $\mathbb{S}(t)$ intersects $\left[-u_{2},-v_{2}\right]$. Define $t_{3}, t_{4}, \ldots$ similarly. Let $\pi_{n}$ be the probability that $t_{n}$ exists, that is, $\mathbb{S}(t)$ meets the lines $\left[u_{1}, v_{1}\right],\left[-u_{2},-v_{2}\right], \ldots,\left[(-1)^{n+1} u_{n},\left(-1^{n+1}\right) v_{n}\right]$ in at least $n$ successive points. We aim to show that $\pi_{n}$ can be calculated from $\pi_{n-1}$ for any $n$ by changing the gradient and intercepts of the lines, so to show the dependence on the underlying parameters $u_{i}$ and $v_{i}$ the notation

$$
\pi_{n}=\pi_{n}\left(u_{1}, v_{1} ; \ldots ; u_{n}, v_{n}\right)
$$

wil be used.

Let $Q$ be the point $\left(t_{n-1}, \mathbb{S}\left(t_{n-1}\right)\right)$ and assume that $n$ is even. The argument for $n$ being odd follows by changing signs. From Figure 1.3.2 we see that starting at $Q$ we must hit a line $\left[-u_{n},-u_{n-1} t n-1-v_{n-1}-u_{n} t_{n-1}-v_{n}\right]$, which has probability, from THEOREM 1.3.30,

$$
\exp \left(-2 u_{n}\left(u_{n-1} t_{n-1}+v_{n-1}+u_{n} t_{n-1}+v_{n}\right)\right)
$$

We can replace this line by any other line that has the same probability, still depending on $t_{n-1}$. Choose the line with slope $-\left(u_{n-1}+u_{n}\right)$ which is 


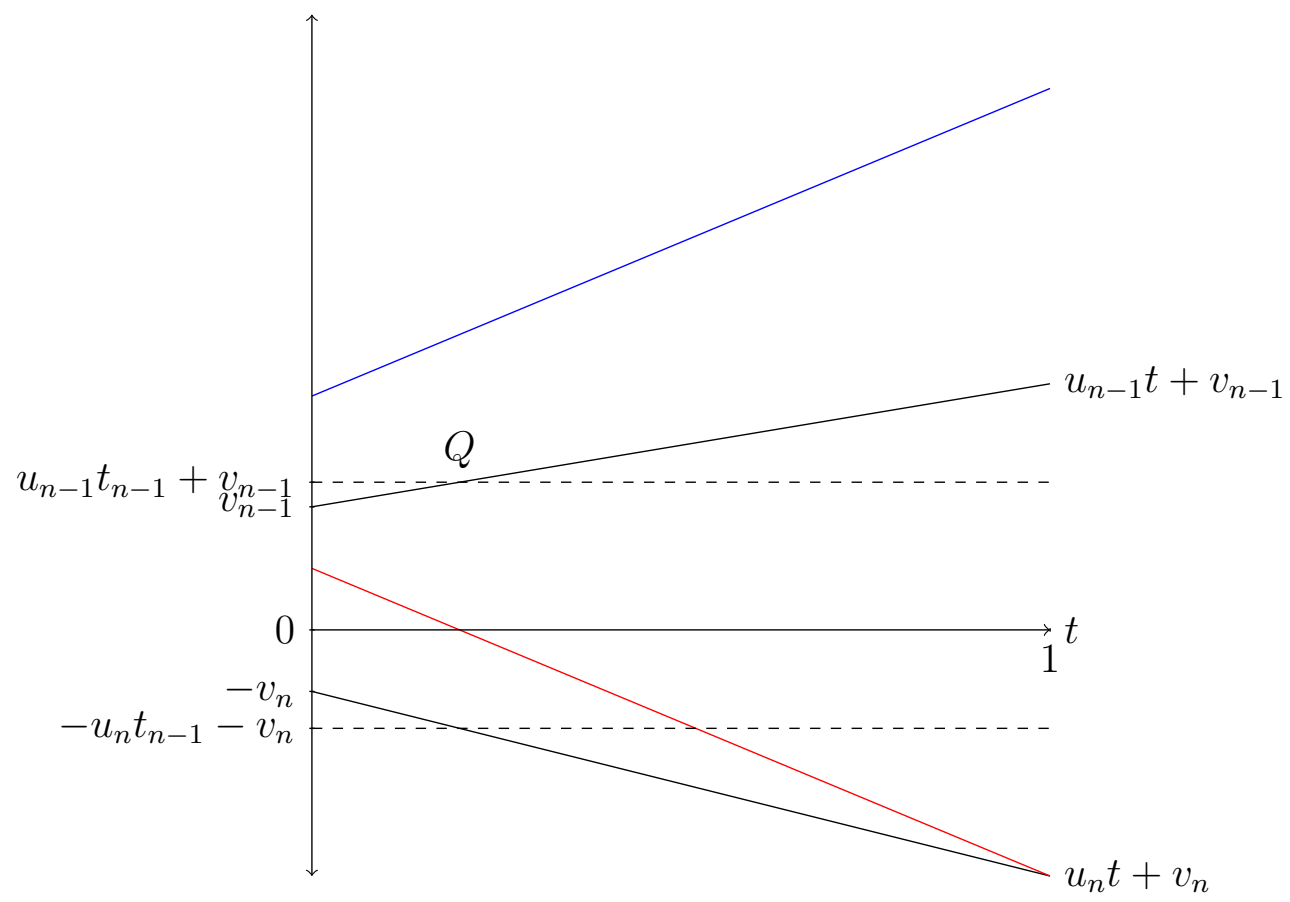

Figure 1.8: Assuming $Q$ is the first intersection of the Brownian motion and the line $u_{n-1} t+v_{n-1}$, the red line is the line with the same probability of intersection as $u_{n} t+v_{n}$ relative to the point $Q$, while the blue line is the red line reflected in the dashed line intersecting $Q$ parallel to the $t$ axis.

$\frac{u_{n}}{u_{n-1}+u_{n-1}}\left(u_{n-1} t_{n-1}+v_{n-1}+u_{n} t_{n-1}+v_{n}\right)$ units below $Q$ at $t_{n-1}$. Using the strong Markov property we may reflect this line in the line parallel to the $t$ axis going through the point $Q$. The reflected line has parameters

$$
\left[u_{n-1}+u_{n}, \frac{u_{n-1} v_{n-1}+u_{n} v_{n}+2 u_{n} v_{n-1}}{u_{n-1}+u_{n}}\right]
$$

so the reflected line is independent of $t_{n-1}$. Therefore

$$
\begin{aligned}
& \pi_{n}\left(u_{1}, v_{1} ; \ldots ; u_{n}, v_{n}\right) \\
& \quad=\pi_{n-1}\left(u_{1}, v_{1} ; \ldots ; u_{n-2}, v_{n-2} ; u_{n-1}+u_{n}, \frac{u_{n-1} v_{n-1}+u_{n} v_{n}+2 u_{n} v_{n-1}}{u_{n-1}+u_{n}}\right)
\end{aligned}
$$

As in Theorem 1.3.29, define events 
$\left\{A_{+}\right\} \equiv\{\mathbb{S}$ crosses the level $a t+b$ for $t \geq 0\}$

$\left\{A_{-}\right\} \equiv\{\mathbb{S}$ crosses the level $-a t-b$ for $t \geq 0\}$

This allows us to inductively define

$\left\{A_{+-}\right\} \equiv\{\mathbb{S}$ crosses the level $a t+b$ then crosses level $-a t-b$ for $t \geq 0\}$

$\left\{A_{-+}\right\} \equiv\{\mathbb{S}$ crosses the level $-a t-b$ then crosses level $a t+b$ for $t \geq 0\}$

and similarly for $A_{+-+}, A_{-+-}, \ldots$

Therefore

$$
\begin{aligned}
\mathbb{P}\left\{\sup _{t \geq 0} \frac{|\mathbb{S}(t)|}{a t+b} \geq 1\right\} & =2\left(\mathbb{P}\left\{A_{+}\right\}-\mathbb{P}\left\{A_{+-}\right\}+\mathbb{P}\left\{A_{+-+}\right\}-\ldots\right) \\
& =2 \sum_{k=1}^{\infty}(-1)^{k+1} \pi_{k}(\underbrace{a, b ; a, b ; \ldots ; a, b}_{k \text { times }}) \\
& =2 \sum_{k=1}^{\infty}(-1)^{k+1} \pi_{1}(k a, k b)
\end{aligned}
$$

Using THEOREM 1.3.30

$$
=2 \sum_{k=1}^{\infty}(-1)^{k+1} \exp \left(-2 k^{2} a b\right)
$$

Theorem 1.3.33: Let $\mathbb{U}$ be Brownian bridge. Then for all $b>0$

$$
\mathbb{P}\left\{\sup _{0 \leq t \leq 1}|\mathbb{U}(t)| \geq b\right\}=2 \sum_{k=1}^{\infty}(-1)^{k+1} \exp \left(-2 k^{2} b^{2}\right)
$$

\section{Proof:}

$$
\mathbb{P}\left\{\sup _{0 \leq t \leq 1}|\mathbb{U}(t)| \geq b\right\}=\mathbb{P}\left\{\sup _{r>0} \frac{|\mathbb{S}(r)|}{1+r} \geq b\right\}
$$


Using THEOREM 1.3.32

$$
=2 \sum_{k=1}^{\infty}(-1)^{k+1} \exp \left(-2 k^{2} b^{2}\right)
$$

\subsection{CENTRAL LIMIT THEOREM FOR EMPIRICAL PROCESSES}

This section outlines central limit theorem type results for the empirical process. We now are dealing with convergence of processes, so we need to consider the theory of convergence in metric spaces to prove the central limit theorem for empirical processes. The first four subsections introduce the theory of convergence in metric spaces, the fifth subsection consider a first application of convergence in metric spaces to prove Donsker's theorem, while showing the existence of the Weiner measure and the final subsection outlines results relevant to empirical processes.

\subsubsection{THEORY OF WEAK CONVERGENCE}

We will begin by outlining the theory of weak convergence. This is a generalisation of convergence in distribution to metric spaces.

Definition 1.4.1: Let $\Omega$ be any set. A metric space $(\Omega, \rho)$ is a pair consisting of a set $\Omega$ and a metric $\rho: \Omega \times \Omega \mapsto \mathbb{R}$ such that:

(a) $\rho(x, y)=0$ iff $x=y$ for all $x, y \in \Omega$.

(b) $\rho(x, y)=\rho(y, x)$ for all $x, y \in \Omega$.

(c) $\rho(x, y) \leq \rho(x, z)+\rho(z, y)$ for all $x, y, z \in \Omega$. 
Definition 1.4.2: A metric space $(\Omega, \rho)$ is complete if every Cauchy (fundmental) sequence in $\Omega$ converges to a limit in $\Omega$.

Definition 1.4.3: A set $B \subseteq \Omega$ is compact if every open covering of $B$ has a finite subcovering.

Let $(S, \rho)$ be a metric space with $\mathcal{S}$ the Borel $\sigma$-field, generated by the open sets.

Definition 1.4.4: A probability measure $\mathbb{P}$ on $(S, \mathcal{S})$ is tight if $\forall \epsilon>0$ there exists compact set $K$ such that $\mathbb{P}\{K\}>1-\epsilon$.

Definition 1.4.5: If probability measures $\mathbb{P}_{n}$ and $\mathbb{P}$ satisfy $\mathbb{P}_{n} f \rightarrow \mathbb{P} f$ for every bounded, continuous real function $f$ on $S$, then $\mathbb{P}_{n}$ converges weakly to $\mathbb{P}$, denoted $\mathbb{P}_{n} \Rightarrow \mathbb{P}$.

Theorem 1.4.6: The following are equivalent:

(i) $\mathbb{P}_{n} \Rightarrow \mathbb{P}$.

(ii) $\mathbb{P}_{n} f \rightarrow \mathbb{P} f$ for all bounded, uniformly continuous $f$.

(iii) $\limsup _{n \rightarrow \infty} \mathbb{P}_{n} F \leq \mathbb{P} F$ for all closed $F$.

(iv) $\liminf _{n \rightarrow \infty} \mathbb{P}_{n} G \geq \mathbb{P} G$ for all open $G$.

(v) $\mathbb{P}_{n}\{A\} \rightarrow \mathbb{P}\{A\}$ for all $\mathbb{P}$-continuity sets $A$ (sets where $\mathbb{P}\{\partial A\}=0$ ).

\section{Proof:}

(i) $\Rightarrow$ (ii) : If it holds for bounded and continuous $f$, then it clearly holds for bounded and uniformly continuous $f$.

(ii) $\Rightarrow$ (iii) : Choose $f(x)=\left(1-\frac{\rho(x, F)}{\epsilon}\right) \vee 0$. It is bounded between 0 and 1 and is uniformly continuous as $|f(x)-f(y)| \leq \frac{\rho(x, y)}{\epsilon}$. Let 
$F_{\epsilon}=\{x: \rho(x, F)<\epsilon\}$. Then it follows, as $x \in F$ implies $f(x)=1$ and $x \notin F_{\epsilon}$ implies $f(x)=0$,

$$
\begin{gathered}
\mathbb{I}_{\{F\}}(x) \leq f(x) \leq \mathbb{I}_{\left\{F_{\epsilon}\right\}}(x) . \\
\limsup _{n \rightarrow \infty} \mathbb{P}_{n} F \leq \limsup _{n \rightarrow \infty} \mathbb{P}_{n} f=\mathbb{P} f \leq \mathbb{P} F_{\epsilon}
\end{gathered}
$$

As $F$ is closed, letting $\epsilon \downarrow 0$ gives the required result.

(iii) $\Rightarrow$ (iv) : Follows immediately by complementation of (iii).

(iv) \& (iii) $\Rightarrow(\mathrm{v})$ : Let $A^{\circ}$ and $A^{-}$be the interior (largest open subset of A) and closure (smallest closed superset of A), it follows that

$$
\begin{aligned}
\mathbb{P}\left\{A^{\circ}\right\} & \leq \liminf _{n \rightarrow \infty} \mathbb{P}_{n}\left\{A^{\circ}\right\} \leq \liminf _{n \rightarrow \infty} \mathbb{P}_{n}\{A\} \\
& \leq \limsup _{n \rightarrow \infty} \mathbb{P}_{n}\{A\} \leq \limsup _{n \rightarrow \infty} \mathbb{P}_{n}\left\{A^{-}\right\} \leq \mathbb{P}\left\{A^{-}\right\}
\end{aligned}
$$

If $A$ is a $\mathbb{P}$-continuity set, then $\mathbb{P}\left\{A^{\circ}\right\}=\mathbb{P}\left\{A^{-}\right\}$and (v) follows.

$(\mathrm{v}) \Rightarrow(\mathrm{i})$ : By linearity we may assume that the bounded $f$ satisfies $0<f<1$. So $\mathbb{P} f=\int_{0}^{1} \mathbb{P}\{f>t\} d t$ and $\mathbb{P}_{n} f=\int_{0}^{1} \mathbb{P}_{n}\{f>t\} d t$. As $f$ is continuous, $\partial\{f>t\} \subseteq\{f=t\}$ and $\{f>t\}$ is a $\mathbb{P}$-continuity set except for countably many $t$. From $(v)$ it follows that $\mathbb{P}_{n}\{f>t\} \rightarrow \mathbb{P}\{f>t\}$ so using the bounded convergence theorem

$$
\mathbb{P}_{n} f=\int_{0}^{1} \mathbb{P}_{n}\{f>t\} d t \rightarrow \int_{0}^{1} \mathbb{P}\{f>t\} d t=\mathbb{P} f
$$

Let $h$ map $S$ to another metric space $S^{\prime}$, with metric $\rho^{\prime}$ and Borel $\sigma$-field $\mathcal{S}^{\prime}$. If $h$ is measurable $\mathcal{S} / \mathcal{S}^{\prime}\left(A^{\prime} \in \mathcal{S}^{\prime}\right.$ implies $\left.A \in \mathcal{S}\right)$, then each $\mathbb{P}$ on $(S, \mathcal{S})$ induces a probability measure $\mathbb{P} h^{-1}$ (defined by $\mathbb{P} h^{-1}\{A\}=\mathbb{P}\left\{h^{-1} A\right\}$ ).

Theorem 1.4.7: If $h$ is continuous, then $\mathbb{P}_{n} \Rightarrow \mathbb{P}$ implies $\mathbb{P}_{n} h^{-1} \Rightarrow \mathbb{P} h^{-1}$. 
Proof: Let $f$ be bounded and continuous on $S^{\prime}$, then as $h$ is continuous, it follows that $f h$ is continuous and bounded on $S$, so by change of variable it follows that

$$
\begin{aligned}
& \int_{S^{\prime}} f(y) d \mathbb{P}_{n} h^{-1}(y)=\int_{S} f(h(x)) d \mathbb{P}_{n}(x) \\
& \stackrel{\mathbb{P}_{n} \Rightarrow \mathbb{P}}{\longrightarrow} \int_{S} f(h(x)) d \mathbb{P}(x)=\int_{S^{\prime}} f(y) d \mathbb{P} h^{-1}(y) .
\end{aligned}
$$

\subsubsection{CONVERGENCE IN DISTRIBUTION}

We are considering mappings $X$ from a probability space $(\Omega, \mathcal{F}, P)$ to a metric space $S . \quad X$ is a random element if it is measurable $\mathcal{F} / \mathcal{S}(S \in \mathcal{S}$ implies $\left.X^{-1} S \in \mathcal{F}\right)$. The distribution of $X$ is the probability measure $\mathbb{P}=P X^{-1}$ on $(S, \mathcal{S})$ defined for $A \in \mathcal{S}$ by

$$
\mathbb{P}\{A\}=P\left\{X^{-1}(A)\right\}=P\{\omega: X(\omega) \in A\} .
$$

This is also called the law of $X$, denoted $\mathcal{L}(X)$.

Definition 1.4.8: A sequence $\left\{X_{n}\right\}_{n=1}^{\infty}$ of random elements converges in distribution to a random element $X$ if $\mathbb{P}_{n} \Rightarrow \mathbb{P}$, where $\mathbb{P}_{n}$ and $\mathbb{P}$ are the distribution functions of $X_{n}$ and $X$. This is denoted $X_{n} \stackrel{d}{\rightarrow} X$ and is also sometimes denoted $X_{n} \Rightarrow X$.

We require $X, X_{1}, X_{2}, \ldots$ to map into the same metric space $(S)$ with the same topology but the underlying probability spaces may all be different. This is because we are concerned with the distributions which the background probability spaces induce on $S$. As

$$
E[f(X)]=\int_{\Omega} f(X(\omega)) d P(\omega)=\int_{S} f(x) d \mathbb{P}(x)=\mathbb{P} f
$$


letting $E_{n}$ and $E$ denote expectation on $\left(\Omega_{n}, \mathcal{F}_{n}, P_{n}\right)$ and $(\Omega, \mathcal{F}, P)$, the background probability spaces for $X_{n}$ and $X$, it follows that $\mathbb{P}_{n} f \Rightarrow \mathbb{P} f$ if and only if $E_{n}\left[f\left(X_{n}\right)\right] \rightarrow E[f(X)]$. Letting $P$ and $E$ refer to probabilities and expectations in the background probability space we are working in, it follows that $X_{n} \stackrel{d}{\rightarrow} X$ if and only if $E\left[f\left(X_{n}\right)\right] \rightarrow E[f(X)]$.

Corollary 1.4.9: The following are equivalent:

(i) $X_{n} \stackrel{d}{\rightarrow} X$.

(ii) $E\left[f\left(X_{n}\right)\right] \rightarrow E[f(X)]$ for all bounded, uniformly continuous $f$.

(iii) $\limsup _{n \rightarrow \infty} P\left\{X_{n} \in F\right\} \leq P\{X \in F\}$ for all closed $F$.

(iv) $\liminf _{n \rightarrow \infty} P\left\{X_{n} \in G\right\} \geq P\{X \in G\}$ for all open $G$.

(v) $P\left\{X_{n} \in A\right\} \rightarrow P\{X \in A\}$ for all $X$-continuity sets $A$ (sets where $P\{X \in \partial A\}=0)$.

Proof: Follow directly from Theorem 1.4.6 applied to the mapping $X$.

Definition 1.4.10: Let $a$ be an element of $S$. If, for all $\epsilon>0$

$$
P\left\{\rho\left(X_{n}, a\right)<\epsilon\right\} \rightarrow 1
$$

then $X_{n}$ converges in probability to $a$. This is denoted $X_{n} \stackrel{P}{\rightarrow} a$

RESULT 1.4.11: $X_{n}$ converges in probability to $a$ if and only if $X_{n} \stackrel{d}{\rightarrow} a$

Proof: Given $X_{n}$ converges in probability to $a$, let $G$ be open and $a \in G$. For $\epsilon$ small enough,

$$
\lim _{n} \inf P\left\{X_{n} \in G\right\} \geq \lim _{n} P\left\{\rho\left(X_{n}, a\right)<\epsilon\right\}=1=P\{a \in G\} .
$$

Using Corollary 1.4.9 (iv), $X_{n} \stackrel{d}{\rightarrow} a$. 
Now let $X_{n} \stackrel{d}{\rightarrow} a$. The set $G_{2}=\{x: \rho(x, a)<\epsilon\}$ is open, so it follows that

$$
\liminf _{n} P\left\{X_{n} \in G_{2}\right\} \geq P\left\{a \in G_{2}\right\}=1
$$

Therefore it follows that $P\left\{\rho\left(X_{n}, a\right)<\epsilon\right\} \rightarrow 1$.

Let $\left(X_{n}, Y_{n}\right)$ be a random element of $S \times S$ and consider the random variable $\rho\left(X_{n}, Y_{n}\right)$ which takes value $\rho\left(X_{n}(\omega), Y_{n}(\omega)\right)$ at point $\omega$.

Theorem 1.4.12: Let $\left(X_{n}, Y_{n}\right)$ be random elements of $S \times S$. If $X_{n} \Rightarrow X$ and $\rho\left(X_{n}, Y_{n}\right) \stackrel{d}{\rightarrow} 0$, then $Y_{n} \Rightarrow X$.

Proof: Let $F_{\epsilon}=\{x: \rho(x, F) \leq \epsilon\}$. Then either $Y_{n} \in F$ and $\rho\left(X_{n}, Y_{n}\right)<\epsilon$ which implies $X_{n} \in F$, or $\rho\left(X_{n}, Y_{n}\right) \geq \epsilon$

$$
\mathbb{P}\left\{Y_{n} \in F\right\} \leq \mathbb{P}\left\{\rho\left(X_{n}, Y_{n}\right) \geq \epsilon\right\}+\mathbb{P}\left\{X_{n} \in F_{\epsilon}\right\}
$$

As $F_{\epsilon}$ is closed, using Corollary 1.4.9 (iii)

$$
\limsup _{n \rightarrow \infty} \mathbb{P}\left\{Y_{n} \in F\right\} \leq \limsup _{n \rightarrow \infty} \mathbb{P}\left\{X_{n} \in F_{\epsilon}\right\} \leq \mathbb{P}\left\{X \in F_{\epsilon}\right\}
$$

If $F$ is closed, then $F_{\epsilon} \downarrow F$ as $\epsilon \downarrow$. Using Corollary 1.4.9 (iii) again completes the proof.

Corollary 1.4.13: Let $\left(X, Y_{n}\right)$ be random elements of $S \times S$. If $\rho\left(X, Y_{n}\right) \stackrel{d}{\rightarrow}$ 0 , then $Y_{n} \stackrel{d}{\rightarrow} X$.

Proof: Choose the $X_{n}$ in Theorem 1.4.12 to be the sequence $X_{n}=X$ for all $n$.

We restrict our attention for the remainder of this subsection to random variables, where $S=\mathbb{R}$. 
Theorem 1.4.14: If $X_{n} \stackrel{d}{\rightarrow} X$, then $E|X| \leq \liminf _{n} E\left|X_{n}\right|$

Proof: By Theorem 1.4.7 $\left|X_{n}\right| \stackrel{d}{\rightarrow}|X|$. Using Corollary 1.4.9(v) $P\left\{\left|X_{n}\right|>t\right\} \rightarrow P\{|X|>t\}$, which is open, so using Corollary 1.4.9 (iv) and Fatou's lemma

$$
\begin{aligned}
E|X| & =\int_{0}^{\infty} P\{|X|>t\} d t \\
& \leq \int_{0}^{\infty} \liminf _{n \rightarrow \infty} P\left\{\left|X_{n}\right|>t\right\} d t \\
& \leq \liminf _{n \rightarrow \infty} \int_{0}^{\infty} P\left\{\left|X_{n}\right|>t\right\} d t=\liminf _{n \rightarrow \infty} E\left|X_{n}\right|
\end{aligned}
$$

\subsubsection{PROHOROV'S THEOREM}

Let $\Pi$ be a family of probability measures on $(S, \mathcal{S})$. We extend the definition of tightness for a probability measure to the family of probability measures in the following way.

Definition 1.4.15: The family $\Pi$ is tight if, for every $\epsilon$ there exists a compact set $K$ such that $\mathbb{P}\{K\}>1-\epsilon$ for every $\mathbb{P}$ in $\Pi$.

Definition 1.4.16: $\Pi$ is relatively compact if every sequence of elements of $\Pi$ contains a weakly convergent subsequence. This means that for every sequence $\left\{\mathbb{P}_{n}\right\}_{n=1}^{\infty}$ there exists a subsequence $\left\{\mathbb{P}_{n_{i}}\right\}_{n=1}^{\infty}$ and a probability measure $Q$, defined on $(S, \mathcal{S})$ but not necessarily an element of $\Pi$, such that $\mathbb{P}_{n_{i}} \stackrel{d}{\rightarrow} Q$ as $i \rightarrow \infty$. We also require that $Q\{S\}=1$, so that probability cannot escape in the limit.

The reason to introduce relative compactness is that on the space $C[0,1]$, the space of continuous functions on the unit interval, relative compactness and convergence of the finite-dimensional distributions implies weak convergence. 
This idea also extends to other spaces. The idea is then to find a way of proving relative compactness. This is done by using Prohorov's theorem.

Definition 1.4.17: A set $A$ is dense in $B$, if $\forall x \in B$ such that $x \in J$ for open $J$, then $J \cap A \neq \emptyset$. This is equivalent to $B \subseteq A^{-}$

Definition 1.4.18: The space $S$ is separable if it contains a countable, dense subset. A subset $M$ of $S$ is separable if there is a countable set $D$ that is dense in $M\left(M \subseteq D^{-}\right)$

Lemma 1.4.19: Suppose that the subset $M$ of $S$ is separable. Then there is a countable class $\mathcal{A}$ of open sets with the property that, if $x \in G \cap M$ for $G$ open, then $x \subseteq A \subseteq A^{-} \subseteq G$ for some $A \in \mathcal{A}$.

Proof: Let $D$ be a countable, dense subset of $M$ and take $\mathcal{A}$ to be the balls $B(d, r)$ for $d \in D$ and $r$ rational, so $\mathcal{A}$ is countable and open. If $x \in G \cap M$ and $G$ is open, choose $\epsilon$ so that $B(x, \epsilon) \subseteq G$, which exists as $G$ is open, then choose $d \in D$ so that $\rho(x, d)<\frac{\epsilon}{2}$, which exists as $D$ is dense in $D$ is dense. Finally choose a rational $r$ so that $\rho(x, d)<r<\frac{\epsilon}{2}$. It immediately follows that $x \subseteq B(d, r) \subseteq B(d, r)^{-} \subseteq B(x, \epsilon) \subseteq G$

Theorem 1.4.20 (Prohorov's Theorem): Let $\Pi$ be a family of probability measures. Then

(i) If $\Pi$ is tight, then $\Pi$ is relatively compact.

(ii) If $\Pi$ is relatively compact and $S$ is separable and complete, then $\Pi$ is tight.

\section{Proof:}

(i) Let $\left\{\mathbb{P}_{n}\right\}_{n=1}^{\infty}$ be a sequence in the tight family $\Pi$. Choose compact sets $K_{j}$ so that $K_{1} \subseteq K_{2} \subseteq K_{3} \cdots$ and $\mathbb{P}_{j}\left\{K_{j}\right\}>1-\frac{1}{j}$. The set 
$\bigcup_{i=1}^{n} K_{i}$ is separable. Therefore we can construct $\mathcal{H}$ consisting of $\emptyset$ and finite unions of the sets $A^{-} \cap K_{j}$ for $j \geq 1$ and $A \in \mathcal{A}$, the class of open sets that exist by LEMMA 1.4.19. Using the diagonal method that can be found in Billingsley (1986), there exists a subsequence $\left\{\mathbb{P}_{n(i)}\right\}_{i=1}^{\infty}$ for which the limit $\alpha(H)=\lim _{i \rightarrow \infty} \mathbb{P}_{n(i)}\{H\}$ exists for each $H$ in the countable class $\mathcal{H}$. Claim that the probability measure is $\mathbb{P}\{G\}=\sup _{H \subset G} \alpha(H)$ for all open sets $G$. If such a measure exists then for $H \subseteq G$ it follows that $\alpha(H) \leq \liminf _{i} \mathbb{P}_{n(i)}\{G\}$, which implies that $\mathbb{P}\{G\} \leq \liminf _{i} \mathbb{P}_{n(i)}\{G\}$, so by TheOREM 1.4.6 $\mathbb{P}_{n(i)} \stackrel{d}{\rightarrow} \mathbb{P}$. The remainder of the proof is to show that $\mathbb{P}$ is a probability measure. It follows as $K_{j}$ has a finite covering of $\mathcal{A}$ sets by compactness, that $K_{j}$ lies in $\mathcal{H}$. Therefore

$$
1 \geq \mathbb{P}\{S\} \geq \sup _{j} \alpha\left(K_{j}\right) \geq \sup _{u}\left(1-\frac{1}{u}\right)=1 .
$$

Some measurability concerns still remain, which are dealt with in Billingsley (1999).

(ii) Let $G_{n}$ be open sets increasing to $S$. First it will be proved that $\forall \epsilon>0$ there is an $n$ such that $\mathbb{P}\left\{G_{n}\right\}>1-\epsilon$ for all $\mathbb{P}$ in $\Pi$. Assume not; then for each $n$ there exists $\mathbb{P}_{n}$ in $\Pi$ with $\mathbb{P}_{n} G_{n} \leq 1-\epsilon$. As $\Pi$ is relatively compact, there is a subsequence $\mathbb{P}_{n(i)} \Rightarrow \mathbb{Q}$ for some probability measure $\mathbb{Q}$. This is impossible as

$$
\mathbb{Q}\left\{G_{n}\right\} \leq \liminf _{i} \mathbb{P}_{n(i)}\left\{G_{n}\right\} \leq \liminf _{i} \mathbb{P}_{n(i)}\left\{G_{n(i)}\right\} \leq 1-\epsilon
$$

but $G_{n} \uparrow S$. From separability there exists a sequence $A_{k_{1}}, A_{k_{2}}, \ldots$ of open balls of radius $\frac{1}{k}$ which cover $S$. Then there exists an $n_{k}$ such that for all $\mathbb{P}$ in $\Pi, \mathbb{P}\left\{\bigcup_{i \leq n_{k}} A_{k i}\right\}>1-\frac{\epsilon}{2^{k}}$ as the sequence $A_{k_{1}}, A_{k_{2}}, \ldots$ covers $S$. The set $A=\bigcap_{k \geq 1} \bigcup_{i \leq n_{k}} A_{k i}$ is totally bounded 
and as the space $S$ is complete, the closure of $A$ is compact. Let $K=A$. Then $\mathbb{P}\{K\}>1-\epsilon$ for all $\mathbb{P}$ in $\Pi$.

Theorem 1.4.21: If the sequence $\left\{\mathbb{P}_{n}\right\}$ is relatively compact and the finitedimensional distributions of $\mathbb{P}_{n}$ converge weakly to the finite-dimensional distributions of $\mathbb{P}$, then $\mathbb{P}_{n} \stackrel{n}{\Rightarrow} \mathbb{P}$

Proof : Let $\mathbb{A} \pi_{t_{1}, \ldots, t_{k}}^{-1}$ represent the finite-dimensional distributions of some probability measure $\mathbb{A}$. As $\left\{\mathbb{P}_{n}\right\}$ is relatively compact, each subsequence $\left\{\mathbb{P}_{m}\right\}$ contains a further subsequence $\left\{\mathbb{P}_{m(i)}\right\}$ converging weakly to some $\mathbb{Q}$. From THEOREM 1.4.7 it follows that $\mathbb{P}_{m(i)} \pi_{t_{1}, \ldots, t_{k}}^{-1} \Rightarrow \mathbb{Q} \pi_{t_{1}, \ldots, t_{k}}^{-1}$ and using the convergence of the finite-dimensional distributions it follows that $\mathbb{Q} \pi_{t_{1}, \ldots, t_{k}}^{-1}=\mathbb{P} \pi_{t_{1}, \ldots, t_{k}}^{-1}$ for all $t_{1}, \ldots, t_{k}$. This implies that $\mathbb{P}_{n} \Rightarrow \mathbb{P}$

\subsubsection{THE SPACE $c$}

We now consider the space $C[0,1]$ (abbreviated to $C$ ) with the uniform topology, with the metric

$$
\rho(x, y)=\|x-y\|=\sup _{0 \leq t \leq 1}|x(t)-y(t)|
$$

Weak convergence does not follow from convergence of finite-dimensional distributions alone, but weak convergence does follow from relative compactness and convergence of finite-dimensional distributions. From Prohorov's theorem we have the following.

Theorem 1.4.22: Let $\mathbb{P}_{n}, \mathbb{P}$ be probability measures on $(C, \mathcal{C})$. If the finitedimensional distributions of $\mathbb{P}_{n}$ converge weakly to the finite-dimensional distributions of $\mathbb{P}$ and if $\{\mathbb{P}\}_{n=1}^{\infty}$ is tight, then $\mathbb{P}_{n} \Rightarrow \mathbb{P}$. 
Proof: Follows immediately from Theorem 1.4.20 and Theorem 1.4.21

To use TheOrem 1.4.22 in $C$, we need to understand relative compactness in $C$. This is the statement of the Arzela-Ascoli theorem. Before the statement of the Arzela-Ascoli theorem, the modulus of continuity is defined.

Definition 1.4.23: The modulus of continuity of an arbitrary function $x$ (not necessarily in $C$ ) on $[0,1]$ is

$$
w_{x}(\delta)=\sup _{|s-t| \leq \delta}|x(s)-x(t)|, \quad 0<\delta \leq 1
$$

Theorem 1.4.24 (Arzela-Ascoli): The set $A$ in $C$ is relatively compact (each sequence in $A$ contains a convergent subsequence) iff

$$
\sup _{x \in A}|x(0)|<\infty
$$

and

$$
\lim _{\delta \rightarrow 0} \sup _{x \in A} w_{x}(\delta)=0
$$

Proof: Let $A$ be relatively compact. As $C$ is a metric space, this is equivalent to $A^{-}$is compact, which implies (1.9). Given fixed $\delta>0$, $\left|w_{x}(\delta)-w_{y}(\delta)\right| \leq 2 \rho(x, y)$, so $w_{x}(\delta)$ is continuous in $x$. As $w_{x}\left(\frac{1}{n}\right)$ is non-increasing in $n$, Dini's theorem applies, which states that for functions that are continuous and monotonically decreasing to 0 , the convergence in uniform on compact sets and (1.10) follows as $A^{-}$is compact.

Now let $A$ satisfy (1.9) and (1.10). Choose $k$ large enough that $\sup _{x \in A} w_{x}\left(\frac{1}{k}\right)$ is finite. Using the triangle inequality

$$
|x(t)| \leq|x(0)|+\sum_{i=1}^{k}\left|x\left(\frac{i t}{k}\right)-x\left(\frac{(i-1) t}{k}\right)\right|
$$


and it follows that

$$
\sup _{0 \leq t \leq 1} \sup _{x \in A}|x(t)|<\infty
$$

We want to use (1.10) and (1.11) to prove that $A$ is relatively compact. Let $\left\{t_{k}\right\}_{k=1}^{\infty}$ be an enumeration of the rationals in [0,1]. Using (1.11) it follows that $\left\{x\left(t_{k}\right)\right\}_{x \in A}$ is bounded for given $k$, so there exists a subsequence (of a given sequence of elements in $A)\left\{x_{n_{1}}\right\}_{n_{1}=1}^{\infty} \in A$ such that $\left\{x_{n_{1}}\left(t_{1}\right)\right\}_{n_{1}=1}^{\infty}$ converges. Continuing for $t_{2}, t_{3}, \ldots$ we find $\left\{x_{n_{2}}\right\}_{n_{2}=1}^{\infty}$ a subsequence of $\left\{x_{n_{1}}\right\}_{n_{1}=1}^{\infty}$ which converges on for $t_{2}$ and $\left\{x_{n_{i}}\right\}_{n_{i}=1}^{\infty}$ a subsequence of $\left\{x_{n_{i-1}}\right\}_{n_{i-1}=1}^{\infty}$ which converges on for $t_{i}$. Therefore the sequence of functions $\left\{x_{n}\right\}_{n=1}^{\infty}$, where $x_{j}$ is the $j$-th function of the sequence $\left\{x_{n_{j}}\right\}_{n_{j}=1}^{\infty}$, converges for rational $t_{k} \in[0,1]$. So there exists an $N$ such that

$$
\left|x_{n}\left(t_{k}\right)-x_{m}\left(t_{k}\right)\right|<\frac{\epsilon}{3} \quad \text { for } m, n \geq N
$$

By (1.10), for given $\epsilon$ and any $t \in[0,1]$ there exists a $t_{k}$ such that $\sup _{x \in A}\left|x(t)-x\left(t_{k}\right)\right|<\frac{\epsilon}{3}$ when $\left|t-t_{k}\right|<\delta$. Further for given $\delta$ we only need a finite number of $t_{k}$, as $\bigcup_{k=1}^{\infty} B\left(t_{k}, \delta\right)$ form an open cover of $[0,1]$, so there is a finite subcover, $B\left(t_{1^{\prime}}, \delta\right), B\left(t_{2^{\prime}}, \delta\right), \ldots, B\left(t_{j^{\prime}}, \delta\right)$. Therefore for any $t \in[0,1]$ we have

$$
\sup _{t \in[0,1]}\left|x_{n}(t)-x_{m}(t)\right| \leq\left|x_{n}(t)-x_{n}\left(t_{k}\right)\right|+\left|x_{n}\left(t_{k}\right)-x_{m}\left(t_{k}\right)\right|+\left|x_{m}(t)-x_{m}\left(t_{k}\right)\right|
$$

So for $m, n$ greater that some finite $N^{\prime}$

$$
\leq \frac{\epsilon}{3}+\frac{\epsilon}{3}+\frac{\epsilon}{3}=\epsilon
$$

So the sequence $\left\{x_{n}\right\}_{n=1}^{\infty}$ Cauchy in $C$ with respect to the uniform metric, therefore the limit is continuous as $C$ is complete. Therefore $A$ is relatively compact. 
Now we want to consider probability measures on $(C, \mathcal{C})$.

Theorem 1.4.25: The sequence of probability measures $\left\{\mathbb{P}_{n}\right\}$ is tight if and only if the following two conditions hold

(a) For all $\eta>0$, there exists $a$ and $N$ such that

$$
\mathbb{P}_{n}\{x:|x(0)| \geq a\} \leq \eta, \quad n \geq N
$$

(b) For all $\eta>0$ and $\epsilon>0$, there exists a $0<\delta<1$ and an $N$ such that

$$
\mathbb{P}_{n}\left\{x: w_{x}(\delta) \geq \epsilon\right\} \leq \eta, \quad n \geq N
$$

(b) is equivalent to

$$
\lim _{\delta \rightarrow 0} \limsup _{n \rightarrow \infty} \mathbb{P}_{n}\left\{x: w_{x}(\delta) \geq \epsilon\right\}=0
$$

Proof: Let $\left\{\mathbb{P}_{n}\right\}$ be tight. For given $\eta$, there is a compact $K$ such that $\mathbb{P}_{n}\{K\}>1-\eta$ for all $n$. By ThEOREM 1.4.24 for large enough $a$ we have $K \subseteq\{x:|x(0)| \leq a\}$ and for small enough $\delta$ we have $K \subseteq\left\{x: w_{x}(\delta) \leq \epsilon\right\}$, so (a) and (b) hold for $N=1$.

Let (a) and (b) hold. We want to show that any given probability measure on $C$ is tight, and then extend this to the sequence of probability measures. Since $C$ is separable, for each $k$ there is a sequence of open $\frac{1}{k}$ balls $A_{k 1}, A_{k 2}, \ldots$ covering $C$. Choose $n_{k}$ large enough so that $\mathbb{P}\left\{\bigcup_{i \leq n_{k}} A_{k i}\right\}>1-\frac{\epsilon}{2 k}$. As $C$ is complete, the totally bounded set $\left\{\bigcap \bigcup_{k \geq 1} A_{i \leq n_{k}}\right\}$ has compact closure $K$, with $\mathbb{P}\{K\}>1-\epsilon$. Therefore using the necessity of the thoerem, (a) and (b) hold for each $\mathbb{P}_{n}$ individually. By choosing $a$ to be the maximum of $a$ that work for the first $j$ elements of $\left\{\mathbb{P}_{n}\right\}$, and choosing $\delta$ to be the mminimum of 
the $\delta$ that work for the first $j$ elements of $\left\{\mathbb{P}_{n}\right\}$, we have that (a) and (b) hold for finitely many elements of $\left\{\mathbb{P}_{n}\right\}$, so we may assume that $N=1$.

Given $\eta$, from (a) we may choose $a$ so that, if $B=\{x:|x(0)| \leq a\}$, then $\mathbb{P}_{n}\{B\} \geq 1-\eta$ for all $n$. Similarly, from (b) we may choose $\delta_{k}$ so that, if $B_{k}=\left\{x: w_{x}\left(\delta_{k}\right)<\frac{1}{k}\right\}$, then $\mathbb{P}_{n}\left\{B_{k}\right\} \geq 1-\frac{\eta}{2^{k}}$ for all $n$. Let $K$ be the closure of $A=B \cap\left(\bigcap_{k} B_{k}\right)$, then $\mathbb{P}_{n}\{K\} \geq 1-2 \eta$ and from ThEOREM 1.4.24 it follow that $A$ is relatively compact and $K$ is compact. Therefore $\left\{\mathbb{P}_{n}\right\}$ is tight.

Theorem 1.4.26: Let $0=t_{0}<t_{1}<\cdots<t_{v}=1$ and

$$
\min _{1<i<v}\left(t_{i}-t_{i-1}\right) \geq \delta
$$

Then for arbitrary $x$,

$$
w_{x}(\delta) \leq 3 \max _{1 \leq i \leq v}\left(\sup _{t_{i-1} \leq s \leq t_{i}}\left|x(s)-x\left(t_{i}\right)\right|\right)
$$

and for arbitrary $\mathbb{P}$,

$$
\mathbb{P}\left\{x: w_{x}(\delta) \geq 3 \epsilon\right\} \leq \sum_{i=1}^{v} \mathbb{P}\left\{x: \sup _{t_{i-1} \leq s \leq t_{i}}\left|x(s)-x\left(t_{i}\right)\right| \geq \epsilon\right\}
$$

Proof: Let $m$ be the maximum in (1.15). If $s$ and $t$ lie in the same interval $\left[t_{i-1}, t_{i}\right]$, then

$$
|x(s)-x(t)| \leq\left|x(s)-x\left(t_{i-1}\right)\right|+\left|x(t)-x\left(t_{i-1}\right)\right| \leq 2 m .
$$

If $s$ and $t$ lie in adjacent intervals, then

$$
|x(s)-x(t)| \leq\left|x(s)-x\left(t_{i-1}\right)\right|+\left|x\left(t_{i}\right)-x\left(t_{i-1}\right)\right|+\left|x(t)-x\left(t_{i-1}\right)\right| \leq 3 m .
$$


Therefore (1.15) holds. Therefore

$$
\begin{aligned}
\mathbb{P}\left\{x: w_{x}(\delta) \geq 3 \epsilon\right\} & \leq \mathbb{P}\left\{x: 3 \max _{1 \leq i \leq v}\left(\sup _{t_{i-1} \leq s \leq t_{i}}\left|x(s)-x\left(t_{i}\right)\right|\right) \geq 3 \epsilon\right\} \\
& \leq \sum_{i=1}^{v} \mathbb{P}\left\{x: \sup _{t_{i-1} \leq s \leq t_{i}}\left|x(s)-x\left(t_{i}\right)\right| \geq \epsilon\right\}
\end{aligned}
$$

\subsubsection{CONSTRUCTION OF THE WEINER MEASURE}

We want to prove that the partial sum process weakly converges to Brownian motion. The first step is to prove the existence of the limiting process, while the second is to prove that this is indeed the correct limiting process. The ideas used in this section can then be generalised to empirical processes, defined on a suitable space to be discussed in the next subsection. This subsection follows Billingsley (1999).

Definition 1.4.27: The Wiener measure, $\mathbb{W}$, is a probability measure on $(C, \mathcal{C})$ that satisfies the following:

(i) Each $x_{t}$ is normally distributed under $W$ with mean 0 and variance $t$

$$
\mathbb{W}\left\{x_{t} \leq a\right\}=\frac{1}{\sqrt{2 \pi t}} \int_{-\infty}^{\alpha} \exp \left(-\frac{u^{2}}{2 t}\right) d u
$$

with $\mathbb{W}\left\{x_{t}=0\right\}=1$

(ii) The stochastic process $\left\{x_{t}: 0 \leq t \leq 1\right\}$ has independent increments under $\mathbb{W}$

The Wiener measure is the measure associated with Brownian motion. What we want to do is prove the existence on $(C, \mathcal{C})$ of a probability measure having a specified finite-dimensional distribution. 
Definition 1.4.28: Given a sequence $\xi_{1}, \xi_{2}, \ldots$ of independent and identically distributed random variables on some probability space having mean 0 and finite, positive variance $\sigma^{2}$. Let $S_{n}=\sum_{i=1}^{n} \xi_{i}$, with $S_{0}=0$, and let $X^{n}(\omega)$ be an element of $C$ defined by

$$
X_{t}^{n}(\omega)=\frac{1}{\sigma \sqrt{n}} S_{\lfloor n t\rfloor}(\omega)+(n t-\lfloor n t\rfloor) \frac{1}{\sigma \sqrt{n}} \xi_{\lfloor n t\rfloor+1}(\omega)
$$

$X^{n}(\omega)$ is the linear interpolation between the values $X_{\frac{i}{n}}^{n}(\omega)=\frac{1}{\sigma \sqrt{n}} S_{i}(\omega)$ defined by the partial sums $S_{i}: i=1,2, \ldots, n$.

Theorem 1.4.29: There exists on $(C, \mathcal{C})$ a probability measure $\mathbb{W}$ having the finite-dimensional distribution specified by

$$
\begin{aligned}
\mathbb{W} & \left\{x_{t_{i}}-x_{t_{i-1}} \leq \alpha_{i}: i=1, \ldots, k\right\} \\
& =\prod_{i=1}^{k} \frac{1}{\sqrt{2 \pi\left(t_{i}-t_{i-1}\right)}} \int_{-\infty}^{\alpha_{i}} \exp \left(-\frac{u^{2}}{2\left(t_{i}-t_{i-1}\right)}\right) d u
\end{aligned}
$$

Proof: The first step is to show that the finite-dimensional distributions of $X^{n}$ converge to the finite-dimensional distributions of $\mathbb{W}$. Using Chebyshev's inequality $(n t-\lfloor n t\rfloor) \frac{1}{\sigma \sqrt{n}} \xi_{\lfloor n t\rfloor+1}(\omega) \stackrel{d}{\rightarrow} 0$ as $n \rightarrow \infty$, and by the central limit theorem $\frac{1}{\sigma \sqrt{n}} S_{\lfloor n t\rfloor}(\omega) \Rightarrow \sqrt{t} N$, where $N$ is the standard normal distribution. Using ThEOREM 1.4.12 $X_{t}^{n} \stackrel{d}{\rightarrow} \sqrt{t} N$. The same argument applied to the differences show that $\left(X_{t_{1}}^{n}, X_{t_{2}}^{n}-X_{t_{1}}^{n}, \ldots, X_{t_{k}}^{n}-X_{t_{k-1}}^{n}\right)$ converge to $\left(N_{1}, N_{2}, \ldots, N_{k}\right)$ with $N_{i}$ having variance $t_{i}-t_{i-1}$. Letting $\mathbb{P}_{n}$ be the distribution on $C$ of $X^{n}$, for each $t_{1}, \ldots, t_{k}$ we have $\mathbb{P}_{n} \pi_{t_{1}, \ldots, t_{k}} \Rightarrow \mathbb{W} \pi_{t_{1}, \ldots, t_{k}}$, so the finite-dimensional distributions converge.

The second step is to show that $X^{n}$ is tight, which implies that $X^{n}$ is relatively compact, so $\mathbb{P}_{n} \stackrel{d}{\rightarrow} \mathbb{W}$. We will show that

$$
\lim _{\lambda \rightarrow 0} \limsup _{n \rightarrow \infty} \lambda^{2} P\left\{\max _{k \leq n}\left|S_{k}\right| \geq \lambda \sigma \sqrt{n}\right\}=0
$$


implies that $X^{n}$ is tight. Using Theorem 1.4.25, we see condition (a) is satisfied as $X_{0}^{n}=0$ for all $n$. For condition (b) we show that, for $\epsilon>0$

$$
\lim _{\delta \rightarrow 0} \limsup _{n \rightarrow \infty} P\left\{w\left(X^{n}, \delta\right) \geq \epsilon\right\}=0 .
$$

Using Theorem 1.4.26, if EquATION (1.14) holds, then

$$
P\left\{w\left(X^{n}, \delta\right) \geq 3 \epsilon\right\} \leq \sum_{i=1}^{v} P\left\{\sup _{t_{i-1} \leq s \leq t_{i}}\left|X_{s}^{n}-X_{t}^{n}\right| \geq \epsilon\right\}
$$

Choosing $t_{i}=\frac{m_{i}}{n}$ for integer $m_{i}$ satisfying $0=m_{0}<m_{1}<\cdots<m_{v}=n$ allows us to evaluate the supremum in 1.18 as a maximum over the carefully chosen $t_{i}$ :

$$
\begin{aligned}
P\left\{w\left(X^{n}, \delta\right) \geq 3 \epsilon\right\} & \leq \sum_{i=1}^{v} P\left\{\max _{m_{i-1} \leq k \leq m_{i}} \frac{\left|S_{k}-S_{m_{i-1}}\right|}{\sigma \sqrt{n}} \geq \epsilon\right\} \\
& =\sum_{i=1}^{v} P\left\{\max _{k \leq m_{i}-m_{i-1}}\left|S_{k}\right| \geq \epsilon \sigma \sqrt{n}\right\} \quad \text { (By independence) }
\end{aligned}
$$

We want to further modify our choice of $m_{i}$ by letting $m_{i}=i m$ for some integer $m$, where $m$ is a function of $n$ and $\delta$. Choose $m$ as $m\lceil n \delta\rceil$ for $i<v$ and choose $v=\left\lceil\frac{n}{m}\right\rceil$, then

$$
m_{v}-m_{v-1} \leq m, \quad v=\left\lceil\frac{n}{m}\right\rceil \stackrel{n}{\rightarrow} \frac{1}{\delta}<\frac{2}{\delta}, \quad \frac{n}{m} \stackrel{n}{\rightarrow} \frac{1}{\delta}>\frac{1}{2 \delta}
$$

so for large $n$

$$
\begin{aligned}
P\left\{w\left(X^{n}, \delta\right) \geq 3 \epsilon\right\} & \leq v P\left\{\max _{k \leq m}\left|S_{k}\right| \geq \epsilon \sigma \sqrt{n}\right\} \\
& \leq \frac{2}{\delta} P\left\{\max _{k \leq m}\left|S_{k}\right| \geq \frac{\epsilon \sigma \sqrt{m}}{\sqrt{2 \delta}}\right\}
\end{aligned}
$$


Let $\lambda=\frac{\epsilon}{\sqrt{2 \delta}}$, so $\lambda \rightarrow \infty$ as $\delta \rightarrow 0$

$$
\leq \frac{4 \lambda^{2}}{\epsilon^{2}} P\left\{\max _{k \leq m}\left|S_{k}\right| \geq \lambda \sigma m\right\}
$$

Using (1.17), for given $\epsilon>0$ and $\eta>0$, there exists a $\lambda$ such that

$$
\frac{4 \lambda^{2}}{\epsilon^{2}} \limsup _{m \rightarrow \infty} P\left\{\max _{k \leq m}\left|S_{k}\right| \geq \lambda \sigma m\right\}<\eta
$$

Letting $m \rightarrow \infty$ implies $n \rightarrow \infty$ and letting $\lambda \rightarrow \infty$ implies $\delta \rightarrow 0$ therefore

$$
\lim _{\delta \rightarrow 0} \limsup _{n \rightarrow \infty} P\left\{w\left(X^{n}, \delta\right) \geq \epsilon\right\}=\lim _{\lambda \rightarrow 0} \limsup _{n \rightarrow \infty} \lambda^{2} P\left\{\max _{k \leq n}\left|S_{k}\right| \geq \lambda \sigma \sqrt{n}\right\}=0
$$

The last step is to show that (1.17) holds. Using Etemadi's inequality implies that

$$
P\left\{\max _{u \leq m}\left|S_{u}\right| \geq \alpha\right\} \leq 3 \max _{u \leq m} P\left\{\left|S_{u}\right| \geq \frac{\alpha}{3}\right\}
$$

so (1.17) holds if

$$
\lim _{\lambda \rightarrow 0} \limsup _{n \rightarrow \infty} \lambda^{2} \max _{k \leq n} P\left\{\left|S_{k}\right| \geq \lambda \sigma \sqrt{n}\right\}=0 .
$$

To prove existence we may use whatever sequence of $\xi_{i}$ we choose, so choose $\xi_{i}$ to be independent standard normal distributions. This means $\frac{S_{k}}{\sqrt{k}}$ also has a normal distribution and as

$$
P\{|N| \geq \lambda\}<\frac{E\left[N^{4}\right]}{\lambda^{4}}=\frac{3}{\lambda^{4}}
$$

we have

$$
P\left\{\left|S_{k}\right| \geq \lambda \sigma \sqrt{n}\right\}=P\{\sqrt{k}|N| \geq \lambda \sigma \sqrt{n}\}<\frac{3}{\lambda^{4} \sigma^{4}}
$$

which implies (1.17), which implies that $X^{n}$ is tight, so using THEorem 1.4.22, we have proved the existence of the Wiener measure.

We have shown that for $\xi_{i}$ standard normal that the Wiener measure exists, 
but it is well-known that $X^{n}$ converges to a Wiener measure for any sequence of independent and identically distributed $\xi_{i}$ with mean 0 and finite, positive variance $\sigma^{2}$. This is the statement of Donsker's theorem, which is proved below.

Theorem 1.4.30 (Donsker's Theorem): If $\xi_{1}, \xi_{2}, \ldots$ are independent and identically distributed with mean 0 and finite, positive variance $\sigma^{2}$, then if $\mathbb{P}_{n}$ is the probability measure associated with $X^{n}$, then $\mathbb{P}_{n} \stackrel{d}{\rightarrow} \mathbb{W}$

Proof: Following from the proof of TheOREM 1.4.29 all that remains is to show that

$$
\lim _{\lambda \rightarrow 0} \limsup _{n \rightarrow \infty} \lambda^{2} \max _{k \leq n} P\left\{\left|S_{k}\right| \geq \lambda \sigma \sqrt{n}\right\}=0
$$

for $\xi_{1}, \xi_{2}, \ldots$ not necessarily standard normal. Using the central limit theorem, if $k_{\lambda}$ is large enough, then for $k_{\lambda} \leq k \leq n$ then

$$
P\left\{\left|S_{k}\right| \geq \lambda \sigma \sqrt{n}\right\} \leq P\left\{\left|S_{k}\right| \geq \lambda \sigma \sqrt{k}\right\}<\frac{3}{\lambda^{4}}
$$

For $k<k_{\lambda}$ we can use Chebyshev's inequality to get

$$
P\left\{\left|S_{k}\right| \geq \lambda \sigma \sqrt{n}\right\} \leq \frac{k_{\lambda} \sigma^{2}}{\lambda^{2} \sigma^{2} n}=\frac{k_{\lambda}}{\lambda^{2} n}
$$

Therefore the maximum in (1.19) is bounded by $\left(\frac{3}{\lambda^{4}}\right) \vee\left(\frac{k_{\lambda}}{\lambda^{2} n}\right)$

\subsubsection{CENTRAL LIMIT THEOREM FOR EMPIRICAL PROCESSES}

We begin by outlining the central limit theorem for empirical processes in $\mathbb{R}$.

Lemma 1.4.31: For $r=1,2, \ldots$ and any choice of $0 \leq t_{1}<t_{2}<\cdots<t_{r} \leq 1$,

$$
\left\langle\mathbb{U}_{n}\left(t_{1}\right), \mathbb{U}_{n}\left(t_{2}\right), \ldots, \mathbb{U}_{n}\left(t_{r}\right)\right\rangle \stackrel{d}{\rightarrow}\left\langle\mathbb{U}\left(t_{1}\right), \mathbb{U}\left(t_{2}\right), \ldots, \mathbb{U}\left(t_{r}\right)\right\rangle
$$




\section{Proof:}

$$
\begin{aligned}
E\left[\mathbb{U}\left(t_{j}\right) \mathbb{U}\left(t_{k}\right)\right]= & \frac{1}{n} E\left[\left(\sum_{i=1}^{n}\left(\mathbb{I}_{\left\{0, t_{j}\right\}}\left(X_{i}\right)-t_{j}\right)\right)\left(\sum_{i=1}^{n}\left(\mathbb{I}_{\left\{0, t_{j}\right\}}\left(X_{i}\right)-t_{j}\right)\right)\right] \\
= & \frac{1}{n} \sum_{i \neq l} E\left[\mathbb{I}_{\left\{0, t_{j}\right\}}\left(X_{i}\right)-t_{j}\right] E\left[\mathbb{I}_{\left\{0, t_{k}\right\}}\left(X_{l}\right)-t_{k}\right] \\
& +\frac{1}{n} \sum_{i=1}^{n} E\left[\left(\mathbb{I}_{\left\{0, t_{j}\right\}}\left(X_{i}\right)-t_{j}\right)\left(\mathbb{I}_{\left\{0, t_{j}\right\}}\left(X_{i}\right)-t_{j}\right)\right] \\
= & E\left[\left(\mathbb{I}_{\left\{0, t_{j}\right\}}\left(X_{1}\right)-t_{j}\right)\left(\mathbb{I}_{\left\{0, t_{j}\right\}}\left(X_{1}\right)-t_{j}\right)\right] \\
= & t_{j} \wedge t_{k}-t_{j} t_{k}
\end{aligned}
$$

Therefore the covariance matrices of $\mathbb{U}_{n}$ and $\mathbb{U}$ are the same, so using the multivariate central limit theorem it follows

$$
\left\langle\mathbb{U}_{n}\left(t_{1}\right), \mathbb{U}_{n}\left(t_{2}\right), \ldots, \mathbb{U}_{n}\left(t_{r}\right)\right\rangle \stackrel{d}{\rightarrow}\left\langle\mathbb{U}\left(t_{1}\right), \mathbb{U}\left(t_{2}\right), \ldots, \mathbb{U}\left(t_{r}\right)\right\rangle
$$

To show weak convergence of the uniform empirical process to Brownian bridge requires an extension to the theory developed so far in this section. The empirical process $\mathbb{U}_{n}(t)$ is not continuous, so we cannot apply the theory of the space $C$ to establish weak convergence. Following BiLlingsley (1999) we define the space $D$ and a metric for $D$.

Definition 1.4.32: Let $D=D[0,1]$ be the space of real functions $x$ on $[0,1]$ that are right-continuous and have left-hand limits:

(i) For $0 \leq t<1, x\left(t^{+}\right)=\lim _{s \downarrow t} x(s)$ exists and $x\left(t^{+}\right)=x(t)$.

(ii) For $0<t \leq 1, x\left(t^{-}\right)=\lim _{s \downarrow t} x(s)$.

Functions satisfying (i) and (ii) are called cadlag functions. 
Definition 1.4.33: Let $\Lambda$ denote the class of strictly increasing, continuous mappings $[0,1] \mapsto[0,1]$. If $\lambda \in \Lambda$, then $\lambda(0)=0$ and $\lambda(1)=1$. Let $\|x\|=\sup _{t}|x(t)|$ (which is bounded $\forall x \in D$ ) and let $I$ be the indentity map on $[0,1]$, then

$$
d(x, y)=\inf _{\lambda \in \Lambda}\{\|\lambda-I\| \vee\|x-y(\lambda)\|\}
$$

This metric defines the Skohorod topology, and we state that under this metric the space $D$ is separable and there exists an equivalent metric under which $D$ is complete. The proof of the following theorem is modified from EInMAHL (1993).

Theorem 1.4.34: In the space $D, \mathbb{U}_{n} \stackrel{d}{\rightarrow} \mathbb{U}$ in $D$, where $\mathbb{U}$ is Brownian bridge.

Proof: For each $m \in \mathbb{N}$, define $A_{m}: D \mapsto D$ by

$$
\left(A_{m}(x)\right)(t)=\left\{\begin{array}{ll}
x\left(\frac{j-1}{m}\right), & \frac{j-1}{m} \leq t<\frac{j}{m} \quad j=1, \ldots, m \\
x(1), & t=1
\end{array}\right\}
$$

Choose $f \in C_{d}^{*}(D)$, the real-valued functions on $D$ that are bounded, uniformly continuous with respect to $d$ and measurable. Then

$$
\begin{aligned}
& 0 \leq\|f\|_{D} \leq C<\infty \quad 0 \leq \delta_{f}(\epsilon)=\sup _{d(x, y)<\epsilon}|f(x)-f(y)|<\infty \\
& \text { On }\left\{d\left(\mathbb{U}_{n}, A_{m}\left(\mathbb{U}_{\ltimes}\right)\right)<\epsilon\right\} \quad \mid f\left(\mathbb{U}_{n}\right)-f\left(A_{m}\left(\left(\mathbb{U}_{n}\right)\right) \mid \leq \delta_{d}(\epsilon)\right. \\
& \text { On }\left\{d\left(\mathbb{U}_{n}, A_{m}\left(\mathbb{U}_{\ltimes}\right)\right) \geq \epsilon\right\} \quad \mid f\left(\mathbb{U}_{n}\right)-f\left(A_{m}\left(\left(\mathbb{U}_{n}\right)\right) \mid \leq 2 C\right.
\end{aligned}
$$


with the same inequalities holding for $\mathbb{U}$. It follows that

$$
\begin{aligned}
\mid E[ & \left.f\left(\mathbb{U}_{n}\right)\right]-E[f(\mathbb{U})] \mid \\
\leq & \left|E\left[f\left(\mathbb{U}_{n}\right)\right]-E\left[f\left(A_{m}\left(\mathbb{U}_{n}\right)\right)\right]\right|+\left|E\left[f\left(A_{m}\left(\mathbb{U}_{n}\right)\right)\right]-E\left[f\left(A_{m}(\mathbb{U})\right)\right]\right| \\
& +\left|E\left[f\left(A_{m}(\mathbb{U})\right)\right]-E[f(\mathbb{U})]\right| \\
\leq & \delta_{d}(\epsilon)+2 C \mathbb{P}\left\{d\left(\mathbb{U}_{n}, A_{m}\left(\mathbb{U}_{n}\right)\right) \geq \epsilon\right\}+\left|E\left[f\left(A_{m}\left(\mathbb{U}_{n}\right)\right)\right]-E\left[f\left(A_{m}(\mathbb{U})\right)\right]\right| \\
& +\delta_{d}(\epsilon)+2 C \mathbb{P}\left\{d\left(\mathbb{U}, A_{m}(\mathbb{U})\right) \geq \epsilon\right\}
\end{aligned}
$$

Letting $\omega_{n}(\iota)$ be the modulus of continuity for the uniform empirical process at stage $n, d\left(\mathbb{U}_{n}, A_{m}\left(\mathbb{U}_{n}\right)\right) \leq \omega_{n}\left(\frac{1}{m}\right)$. For $0<\epsilon \leq \frac{1}{2}$, using THEOREM 1.1.29

$$
\begin{aligned}
\mathbb{P}\left\{d\left(\mathbb{U}_{n}, A_{m}\left(\mathbb{U}_{n}\right)\right) \geq \epsilon\right\} & \leq \mathbb{P}\left\{\omega_{n}\left(\frac{1}{m}\right) \geq \epsilon\right\} \\
& \leq C^{\prime} m \exp \left(\frac{-(1-\epsilon) \epsilon^{2} m}{2} \psi\left(\frac{\epsilon m}{\sqrt{n}}\right)\right)
\end{aligned}
$$

Therefore, using Definition 1.1.23,

$$
\limsup _{n \rightarrow \infty} \mathbb{P}\left\{d\left(\mathbb{U}_{n}, A_{m}\left(\mathbb{U}_{n}\right)\right) \geq \epsilon\right\} \leq C^{\prime} m \exp \left(\frac{1}{2}(1-\epsilon) \epsilon^{2} m\right) \rightarrow 0
$$

as $m \rightarrow \infty$. As the sample paths of $\mathbb{U}$ are uniformly continuous on $[0,1]$, we have $d\left(\mathbb{U}, A_{m}(\mathbb{U})\right) \rightarrow 0$ almost surely as $m \rightarrow \infty$. Therefore, for all $\epsilon>0$,

$$
\mathbb{P}\left\{d\left(\mathbb{U}, A_{m}(\mathbb{U})\right) \geq \epsilon\right\} \rightarrow 0
$$

as $m \rightarrow \infty$.

Finally from Lemma 1.4.31 it follows that $E\left[f\left(A_{m}\left(\mathbb{U}_{n}\right)\right)\right]=E\left[f\left(A_{m}(\mathbb{U})\right)\right]$, therefore $E\left[f\left(\mathbb{U}_{n}\right)\right] \rightarrow E[f(\mathbb{U})]$ and by CoRollary 1.4.9, $\mathbb{U}_{n} \stackrel{d}{\rightarrow} \mathbb{U}$.

The previous theorem can also be proved by similar ideas used for proving Donsker's theorem in $C$, by first deriving a theorem which generalises ArzelaAscoli to $D$, then deriving a probabilistic equuivalent and showing that the uniform empirical process satisfies these conditions. This approach is taken in 


\section{Billingsley (1999)}

For the remaider of this subsection we are considering the $\mathcal{F}$-indexed empirical process $\mathbb{G}_{n}$ introduced in SECTION 1.1.3. The remainder of this sections outlines extensions to the function parametric empirical process following VAN DER VAART \& WeLLner (1996). Let $f \in \mathcal{F}$, then we consider the map

$$
f \mapsto \mathbb{G}_{n} f=\sqrt{n}\left(\mathbb{P}_{n}-\mathbb{P}\right) f=\frac{1}{\sqrt{n}} \sum_{i=1}^{n}\left(f\left(X_{i}\right)-\int f d \mathbb{P}\right)
$$

For a given $f \in \mathcal{F}$ it follows from the central limit theorem, if $\mathbb{P} f^{2}<\infty$

$$
\mathbb{G}_{n} f \stackrel{d}{\rightarrow} N\left(0, \mathbb{P}(f-\mathbb{P} f)^{2}\right)
$$

The aim is to make this statement uniform over $\mathcal{F}$, that is,

$$
\left\|\mathbb{G}_{n}-\mathbb{G}\right\|_{\mathcal{F}}=\sup _{f \in \mathcal{F}}\left|\left(\mathbb{G}_{n}-\mathbb{G}\right) f\right| \rightarrow 0
$$

where $\mathbb{G}$ is an appropriate limiting process. It is assumed that $\sup _{f \in \mathcal{F}}|f(x)-P f|<$ $\infty$ for all $x$, which implies that $\left\|\mathbb{G}_{n}\right\|_{\mathcal{F}}<\infty$, so $\mathbb{G}_{n}$ is a bounded functional on the space $\mathcal{F}$.

Definition 1.4.35: Let $T$ be a metric space. Then $l^{\infty}(T)$ is defined to be the set of all uniformly bounded real functions on $T$. So for $f \in l^{\infty}(T)$, $\sup _{t \in T}|f(t)|<\infty$

This means that the empirical process $\left\{\mathbb{G}_{n} f: f \in \mathcal{F}\right\}$ is an element of $l^{\infty}(\mathcal{F})$. We consider the nature of the limit distribution $\mathbb{G}$ by first considering the marginal distributions. Let $f_{1}, f_{2}, \ldots, f_{k} \in \mathcal{F}$ be any finite set of functions. If $f_{1}, f_{2}, \ldots, f_{k}$ are square integrable, then it follows from the multivariate 
central limit theorem that

$$
\left(\mathbb{G}_{n} f_{1}, \mathbb{G}_{n} f_{2}, \ldots, \mathbb{G}_{n} f_{k}\right) \stackrel{d}{\rightarrow} N(\mathbf{0}, \boldsymbol{\Sigma})
$$

where

$$
\boldsymbol{\Sigma}=\left[\begin{array}{cccc}
\mathbb{P}\left(f_{1}-\mathbb{P} f_{1}\right)^{2} & \mathbb{P} f_{1} f_{2}-\mathbb{P} f_{1} \mathbb{P} f_{2} & \cdots & \mathbb{P} f_{1} f_{k}-\mathbb{P} f_{1} \mathbb{P} f_{k} \\
\mathbb{P} f_{1} f_{2}-\mathbb{P} f_{1} \mathbb{P} f_{2} & \mathbb{P}\left(f_{2}-\mathbb{P} f_{2}\right)^{2} & \cdots & \mathbb{P} f_{2} f_{k}-\mathbb{P} f_{2} \mathbb{P} f_{k} \\
\vdots & \vdots & \ddots & \vdots \\
\mathbb{P} f_{1} f_{k}-\mathbb{P} f_{1} \mathbb{P} f_{k} & \mathbb{P} f_{2} f_{k}-\mathbb{P} f_{2} \mathbb{P} f_{k} & \cdots & \mathbb{P}\left(f_{k}-\mathbb{P} f_{k}\right)^{2}
\end{array}\right]
$$

At this stage if a limit process exists, then $\{\mathbb{G} f: f \in \mathcal{F}\}$ must be a zero-mean Gaussian process with covariance

$$
E\left[\mathbb{G} f_{1} \mathbb{G} f_{2}\right]=\mathbb{P} f_{1} f_{2}-\mathbb{P} f_{1} \mathbb{P} f_{2},
$$

which is the $\mathbb{P}$-Brownian bridge. This requires tightness to establish $\mathbb{G}_{n} \stackrel{d}{\rightarrow} \mathbb{G}$ in $l^{\infty}(\mathcal{F})$, and such a class $\mathcal{F}$ for which this convergence is true is called a Donsker class.

Sufficient conditions for a class to be Donsker depend on the entropy numbers of the class, introduced in SECTION 1.1.3. A finite class of square integrable functions is always Donsker, while the class of all square integrable, uniformly bounded functions is almost never Donsker. It is shown in VAN DER VAART \& WeLlner (1996) that

$$
\int_{0}^{\infty} \sqrt{\log N_{[]}\left(\epsilon, \mathcal{F}, L_{2}(\mathbb{P})\right)} d \epsilon<\infty
$$

is a sufficient condition for a class $\mathcal{F}$ to be $\mathbb{P}$-Donsker. To arrive at this requires an extension of the theory of weak convergence outlined so far ot the space $l^{\infty}(\mathcal{F})$.

We would like to use the theory which has been developed so far, but an element of $l^{\infty}$ is not necessarily continuous, so we cannot use the Arzela-Ascoli theorem used in $C$. However we know that Prohorov's theorem still applies, so 
the idea is to characterise tightness in $l^{\infty}$ in a similar way to the Arzela-Ascoli theorem. This is the idea of asymptotic equicontinuity. The condition which ensures asymptotic equicontinuity for $\mathbb{G}_{n}$ is, for every $\epsilon>0$,

$$
\lim _{\delta \downarrow} \limsup _{n \rightarrow \infty} \mathbb{P}\left\{\sup _{\rho_{\mathbb{P}}(f-g)<\delta}\left|\mathbb{G}_{n}(f-g)\right|>\epsilon\right\}=0
$$

where $\rho_{\mathbb{P}}(f)=\left(\mathbb{P}(f-\mathbb{P} f)^{2}\right)^{\frac{1}{2}}$ is a seminorm.

Notice the similarity between the definition of asymptotic equicontinuity and condition (b) of TheOREM 1.4.25. This leads to the following theorem:

Theorem 1.4.36: A class $\mathcal{F}$ is Donsker if and only if $\mathcal{F}$ is totally bounded in $\mathcal{L}_{2}(\mathbb{P})$ and is asymptotically equicontinuous.

Proof: Can be found in, for example, van Der VaART \& Wellner (1996). 


\section{Collection of Research Problems}

\subsection{FOURIER ANALYSIS OF MIXTURES}

\subsubsection{CHARACTERISTIC FUNCTIONS}

Definition 2.1.1: Let $X$ be a random variable with distribution function $F$. The characteristic function of $X$ (or $F$ ) is the function $\psi$ defined for real $t$ by

$$
\psi(t)=E[\exp (i t X)]=\int_{-\infty}^{\infty} e^{i t x} d F(x)
$$

It can equivalently be defined as

$$
\psi(t)=u(t)+i v(t)
$$

where

$$
u(t)=\int_{-\infty}^{\infty} \cos (t x) d F(x) \quad v(t)=\int_{-\infty}^{\infty} \sin (t x) d F(x)
$$

For distributions $F$ with a density $f$ this is

$$
\psi(t)=\int_{-\infty}^{\infty} e^{i t x} f(x) d x
$$

Lemma 2.1.2: Let $\psi(t)$ be the characteristic function of the random variable $X$ with distribution $F$. Then

1. $\psi(t)$ is uniformly continuous

2. For constants $a, b, a X+b$ has the characteristic function

$$
E[\exp (i t(a X+b))]=e^{i b t} \psi(a t)
$$

\section{Proof:}


1. It is useful to note that $\left|e^{i t x}\right|=1$, where $|()$.$| denotes the complex$ modulus. Therefore

$$
\begin{aligned}
\left|e^{i t(x+h)}-e^{i t x}\right| & \leq\left|e^{i t x}\right|\left|e^{i t h}-1\right| \\
& =\left|e^{i t h}-1\right| \\
& =\left|i t h+\frac{(i t h)^{2}}{2 !}+\ldots\right|=(h t)^{2}+o\left(h^{3}\right)
\end{aligned}
$$

So for $h$ sufficiently small this tends to 0 . Therefore $\psi(t)$ is uniformly continuous.

2 .

$$
E[\exp (i t(a X+b))]=e^{i b t} E[\exp (i t(a X))]=e^{i b t} E[\exp (i(a t) X)]=e^{i b t} \psi(a t)
$$

Lemma 2.1.3: Let $X_{1}$ and $X_{2}$ be independent random varaibles. Then

$$
E\left[\exp \left(i t\left(X_{1}+X_{2}\right)\right)\right]=\psi_{X_{1}}(t) \psi_{X_{2}}(t)
$$

where $\psi_{X_{i}}(t)$ represents the characteristic function of the random variable $X_{i}$.

\section{Proof :}

$$
\begin{aligned}
E\left[\exp \left(i t\left(X_{1}+X_{2}\right)\right)\right] & =E\left[\exp \left(i t X_{1}\right) \exp \left(i t X_{2}\right)\right] \\
& =E\left[\exp \left(i t X_{1}\right)\right] E\left[\exp \left(i t X_{2}\right)\right] \quad \text { (By independence) } \\
& =\psi_{X_{1}}(t) \psi_{X_{2}}(t)
\end{aligned}
$$

Corollary 2.1.4: 1 . Let $X_{1}, X_{2}, \ldots, X_{n}$ be a finite sequence of independent random variables, with distribution functions $F_{1}, F_{2}, \ldots, F_{n}$ 
respectively. Then if

$$
U=\sum_{i=1}^{n} X_{i}
$$

the characteristic function of $U$ is

$$
E[\exp (i t U)]=\prod_{i=1}^{n} \psi_{X_{i}}(t)
$$

2. A characteristic function is real iff $X$ is symmetric.

\section{Proof:}

1. The result follows directly Lemma 2.1.3.

2. Assuming that the characteristic function uniquely defines a distribution function

$$
\psi(t) \in \mathbb{R} \Leftrightarrow \psi(t)=\overline{\psi(t)} \Leftrightarrow \psi_{X}(t)=\psi_{-X}(t)
$$

Therefore a characteristic function is real iff $X$ is symmetric.

EXAMPLE: Let $X$ be the standard normal distribution, with density

$$
f(x)=\frac{1}{\sqrt{2 \pi}} \exp \left(\frac{-x^{2}}{2}\right) \quad-\infty<x<\infty
$$


The characteristic function can be obtained by

$$
\begin{aligned}
E[\exp (i t X)] & =\int_{-\infty}^{\infty} \exp (i t x) \frac{1}{\sqrt{2 \pi}} \exp \left(\frac{-x^{2}}{2}\right) d x \\
& =\exp \left(\frac{-t^{2}}{2}\right) \int_{-\infty}^{\infty} \frac{1}{\sqrt{2 \pi}} \exp \left(\frac{-(x-i t)^{2}}{2}\right) d x \\
& =\exp \left(\frac{-t^{2}}{2}\right) \int_{-\infty}^{\infty} \frac{1}{\sqrt{2 \pi}} \exp \left(\frac{-(y)^{2}}{2}\right) d y \quad(\text { Let } y=x-i t) \\
& =\exp \left(\frac{-t^{2}}{2}\right)
\end{aligned}
$$

(As the above integral is the normal density over $\mathbb{R}$ )

EXAMPLE: Let $X$ be the uniform distribution on $(a, b)$ for $a<b$, with density

$$
f(x)= \begin{cases}\frac{1}{b-a} & \mathrm{a}<\mathrm{x}<\mathrm{b} \\ 0 & \text { otherwise }\end{cases}
$$

The characteristic function is

$$
\begin{aligned}
E[\exp (i t X)] & =\int_{a}^{b} \exp (i t x) \frac{1}{b-a} d x \\
& =\left.\frac{1}{i t(b-a)} \exp (i t x)\right|_{a} ^{b} \\
& =\frac{\exp (i t b)-\exp (i t a)}{i t(b-a)}
\end{aligned}
$$

Theorem 2.1.5: Let $\psi$ be the characteristic function of the distribution $F$ and suppose that $\psi \in L$. Then $f$ has bounded continuous density $f$ given by

$$
f(x)=\frac{1}{2 \pi} \int_{-\infty}^{\infty} e^{-i t x} \psi(t) d t
$$

\section{Proof :}

$$
\begin{aligned}
e^{-i t x} \psi(t) & =\int_{-\infty}^{\infty} e^{i t(y-x)} d F(y) \\
\Rightarrow \int_{-\infty}^{\infty} e^{-i t x} \psi(t) d G(t) & =\int_{-\infty}^{\infty} \int_{-\infty}^{\infty} e^{i t(y-x)} d F(y) d G(t)
\end{aligned}
$$


Reversing the order of integration and letting $\gamma(t)$ denote the characteristic function of $G$

$$
=\int_{-\infty}^{\infty} \gamma(y-x) d F(y)
$$

Define a function

$$
N(x)=\frac{1}{\sqrt{2 \pi}} \exp \left(\frac{-x^{2}}{2}\right)
$$

and let $G$ be the normal distribution with variance $a^{-2}$. This implies that $g(x)=a N(a x)$ and $\gamma(x)=\sqrt{2 \pi} N\left(\frac{x}{a}\right)$

$$
\begin{aligned}
\Rightarrow \int_{-\infty}^{\infty} e^{-i t x} \psi(t) g(t) d t & =\int_{-\infty}^{\infty} \sqrt{2 \pi} N\left(\frac{y-x}{a}\right) \\
\Rightarrow \frac{1}{2 \pi} \int_{-\infty}^{\infty} e^{-i t x} \psi(t) \exp \left(-\frac{a^{2} t^{2}}{2}\right) d t & =\int_{-\infty}^{\infty} \frac{1}{a} N\left(\frac{x-t}{a}\right) d F(y)
\end{aligned}
$$

The right hand side of the above expression is the convolution of $F$ with a normal distribution with mean 0 and variance $a^{2}$. Therefore

$$
f(x)=\lim _{a \downarrow 0}\left\{\int_{-\infty}^{\infty} \frac{1}{a} N\left(\frac{x-t}{a}\right) d F(x)\right\}
$$

On the other hand, taking the limit of the left hand side gives

$$
\lim _{a \downarrow 0}\left\{\frac{1}{2 \pi} \int_{-\infty}^{\infty} e^{-i t x} \psi(t) e^{-\frac{a^{2} t^{2}}{2}} d t\right\}=\frac{1}{2 \pi} \int_{-\infty}^{\infty} e^{i t x} \psi(t) d t
$$

Therefore

$$
f(x)=\frac{1}{2 \pi} \int_{-\infty}^{\infty} e^{i t x} \psi(t) d t
$$

Theorem 2.1.6: Let $X$ be a random variable with distribution function $F$ 
and characteristic function $\psi(t)$. For $a<b$

$$
F(b)-F(a)=\frac{1}{2 \pi} \int_{-\infty}^{\infty} \psi(t) \frac{e^{-i a t}-e^{-i b t}}{i t} d t
$$

Proof: Consider the convolution of $F$ with a uniform random variable $U$ distributed over the interval $(-b,-a)$, which is independent of $X$. Let $g$ denote the density of this convolution. By conditioning on the value of $X$ it is seen that

$$
g(x)=\int_{x+a}^{x+b} \frac{1}{b-a} d F(x)=\frac{F(x+b)-F(x+a)}{b-a}
$$

Let $\chi(t)$ be the characteristic function of $G$. As $U$ is independent of $X$,

$$
\chi(t)=\psi(t)\left(\int_{-b}^{-a} \frac{e^{i t x}}{b-a} d x\right)=\psi(t) \frac{e^{-i a t}-e^{-i b t}}{i t(b-a)}
$$

Using Theorem 2.1.5 we know that

$$
g(x)=\frac{1}{2 \pi} \int_{-\infty}^{\infty} \chi(t) e^{-i t x} d t
$$

Therefore

$$
\begin{aligned}
\frac{F(x+b)-F(x+a)}{b-a} & =g(x) \\
& =\frac{1}{2 \pi} \int_{-\infty}^{\infty} \chi(t) e^{-i t x} d t \\
& =\frac{1}{2 \pi} \int_{-\infty}^{\infty} \psi(t) \frac{e^{-i a t}-e^{-i b t}}{i t(b-a)} e^{-i t x} d t
\end{aligned}
$$

Let $x=0$ and cancel the $(b-a)^{-1}$ term on each side to get

$$
F(b)-F(a)=\frac{1}{2 \pi} \int_{-\infty}^{\infty} \psi(t) \frac{e^{-i a t}-e^{-i b t}}{i t} d t
$$




\subsubsection{MIXTURES}

Definition 2.1.7: Let $F_{1}, F_{2}, \ldots$ be probability distributions with characteristic functions $\psi_{1}(t), \psi_{2}(t), \ldots, \psi_{n}(t)$. If $p_{i} \geq 0$ and $\sum_{i=1}^{n} p_{i}=1$, then the mixture is defined as

$$
U=\sum_{i=1}^{n} p_{i} F_{i}
$$

Lemma 2.1.8: The mixture is a probability distribution with characteristic function

$$
\psi_{U}(t)=\sum_{i=1}^{n} p_{i} \psi_{F_{i}}(t)
$$

\section{Proof:}

$$
\begin{aligned}
E[\exp (i t x)] & =\int_{-\infty}^{\infty} \exp (i t x) d U(x) \\
& =\int_{-\infty}^{\infty} \exp (i t x)\left(\sum_{i=1}^{n} p_{i} d F_{i}(x)\right) \\
& =\sum_{i=1}^{n} p_{i}\left(\int_{-\infty}^{\infty} \exp (i t x) d F_{i}(x)\right) \\
& =\sum_{i=1}^{n} p_{i} \psi_{F_{i}}(t)
\end{aligned}
$$

Let $t=0$ and $\psi_{U}(0)=1$, which implies that the mixture is a valid probability distribution.

This leads to an interesting problem. Given a mixture of normal distributions with parameters $\mu_{i}$ and $\sigma_{i}^{2}$, if the characteristic function of the mixture was divided by a factor of $\exp \left(-\frac{\sigma_{0}^{2}}{2}\right)$, where $\sigma_{0}^{2}<\min _{i=1, \ldots, n}\left\{\sigma_{i}^{2}\right\}$, can we "remove the background noise' to find the means of the underlying distributions. This corresponds to removing a normal component of mean 0 and variance $\sigma_{0}^{2}$ from each of the components of the mixture. 
This was implemented using numerical methods to construct the characteristic function and the inverse of the characteristic function after division by $\exp \left(-\frac{\sigma_{0}^{2}}{2}\right)$. As the characteristic function is a complex integral, it was evaluated by approximating the real integral and imaginary integral seperately. Let $\Delta=\frac{b_{x}-a_{x}}{n}$, where $\left[a_{x}, b_{x}\right]$ is the interval over the real axis for which the mixture distribution $(X)$ is going to be numerically approximated. Let $a_{x}=x_{0}, x_{k}=x_{k-1}+\Delta$ for $k=1, \ldots, n$. Then

$$
\int_{-\infty}^{\infty} \exp (i t x) d F(x)=\int_{-\infty}^{\infty} \cos (t x) d F(x)+i \int_{-\infty}^{\infty} \sin (t x) d F(x)
$$

So we approximate as folllows

$$
\begin{aligned}
\int_{-\infty}^{\infty} \cos (t x) d F(x) & \approx \sum_{i=0}^{n} \cos \left(t x_{i}\right) f\left(x_{i}\right) \Delta \\
\int_{-\infty}^{\infty} \sin (t x) d F(x) & \approx \sum_{i=0}^{n} \sin \left(t x_{i}\right) f\left(x_{i}\right) \Delta
\end{aligned}
$$

where $f(x)$ is the density of the mixture. This gives an expression for the real and imaginary parts of the characteristic function as a function of $t$, so we must also evaluate these expressions over a set of values for $t$. Let $\left[a_{t}, b_{t}\right]$ be the interval where the characteristic function is going to be numerically evaluated on. Then $\Delta_{t}=\frac{b_{t}-a_{t}}{n}$ and $a_{t}=t_{0}, t_{k}=t_{k-1}+\Delta_{t}$ for $k=1, \ldots, n$. So this sum is evaluated over the set of values for $t_{i}$.

After this has been done, every value of the characteristic function is divided by $\exp \left(-\frac{\sigma_{0}^{2} t^{2}}{2}\right)$. Because this is very large (for $|t| \geq 3$ ) and the error involved in numerically evaluating the integrals is significant when multiplying by such a large value, the characteristic function needs to be smoothed by saying any real or imaginary part with modulus $<\epsilon$ is now $=0$, for $\epsilon$ sensible compared to the value of $\sigma_{0}^{2}$. This is a sensible procedure as the error in evaluating the integral for the characteristic function is about $10^{-17}$, so removing these terms is necessary when $t \geq 3$. As we know that the density is a function in $\mathbb{R}$, we may simplify the inversion formula in Theorem 2.1.5 and approximate the 
inversion by

$$
f^{\prime}(x) \approx \frac{1}{2 \pi} \sum_{i=1}^{n}\left(\cos \left(t_{i} x\right) \mathcal{R}\left(\psi\left(t_{i}\right)\right) \Delta_{t}+\sin \left(t_{i} x\right) \mathcal{I}\left(\psi\left(t_{i}\right)\right) \Delta_{t}\right)
$$

where $\mathcal{R}()$ is the real part and $\mathcal{I}()$ is the imaginary part. Results are provided in the figures at the end of this section.

The last mixture distribution considered in this talk is the mixture of Cauchy random variables. Consider the artificial example of a number of light sources placed a given perpendicular distance from a screen. If a light is randomly chosen and then emits a light ray at a uniformly generated angle (on $(-\pi, \pi)$ ), then the resulting positions where the light rays hit the screen will have a distribution which is a mixture of Cauchy distributions. The modifications to the previous method that are needed to deal with the Cauchy distribution are to used the Cauchy distribution function instead of the normal and to multiply by $\exp (\gamma|t|)$ instead of $\exp \left(\frac{\sigma^{2} t^{2}}{2}\right)$, the characteristic function of the Cauchy distribution with scale parameter $\gamma$ (the characteristic function of the Cauchy distribution with location parameter $\mu$ and scale parameter $\gamma$ is $\exp (i \mu t-\gamma|t|)$, so the division of a central Cauchy distribution with parameter $\gamma^{\prime}<\gamma$ will give a Cauchy distribution with a reduced scale parameter). Results are provided in the figures at the end of this section. 

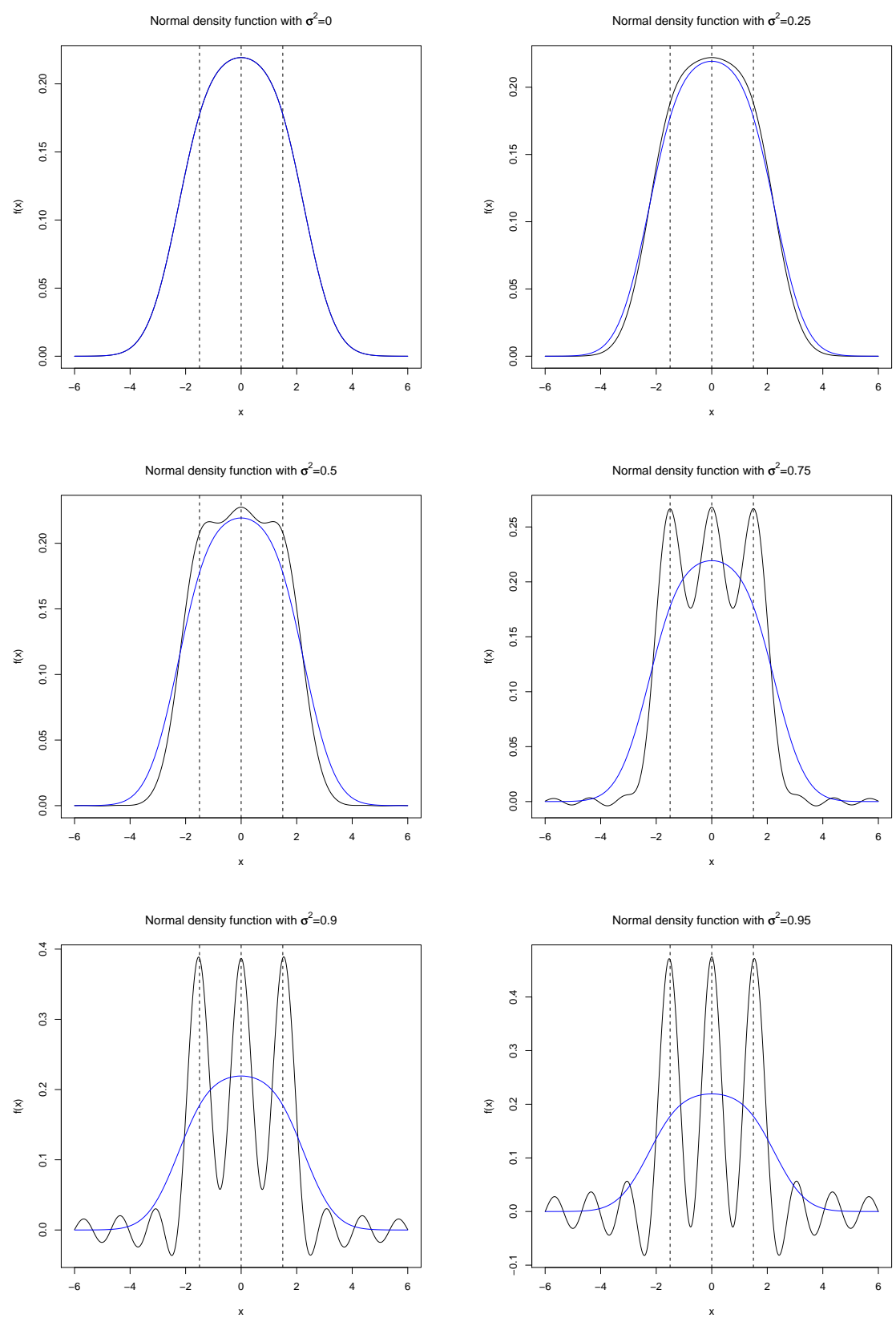

FIGURE 2.1: Mixture of normal densities with $\mu_{1}=-1.5, \mu_{2}=0, \mu_{3}=1.5$ and $\sigma_{i}^{2}=1$. 

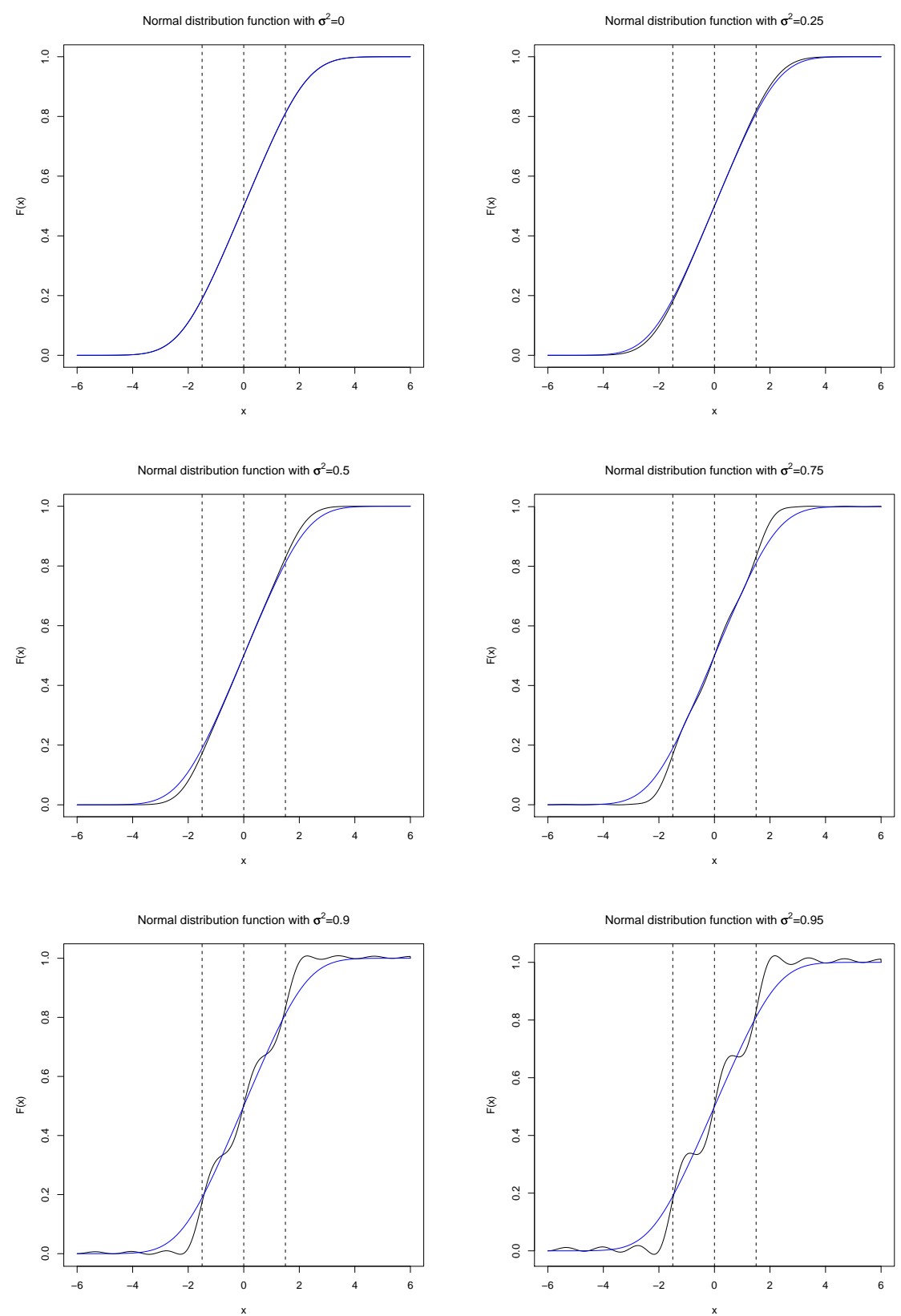

FiguRE 2.2: Mixture of normal distributions with $\mu_{1}=-1.5, \mu_{2}=0, \mu_{3}=1.5$ and $\sigma_{i}^{2}=1$. 

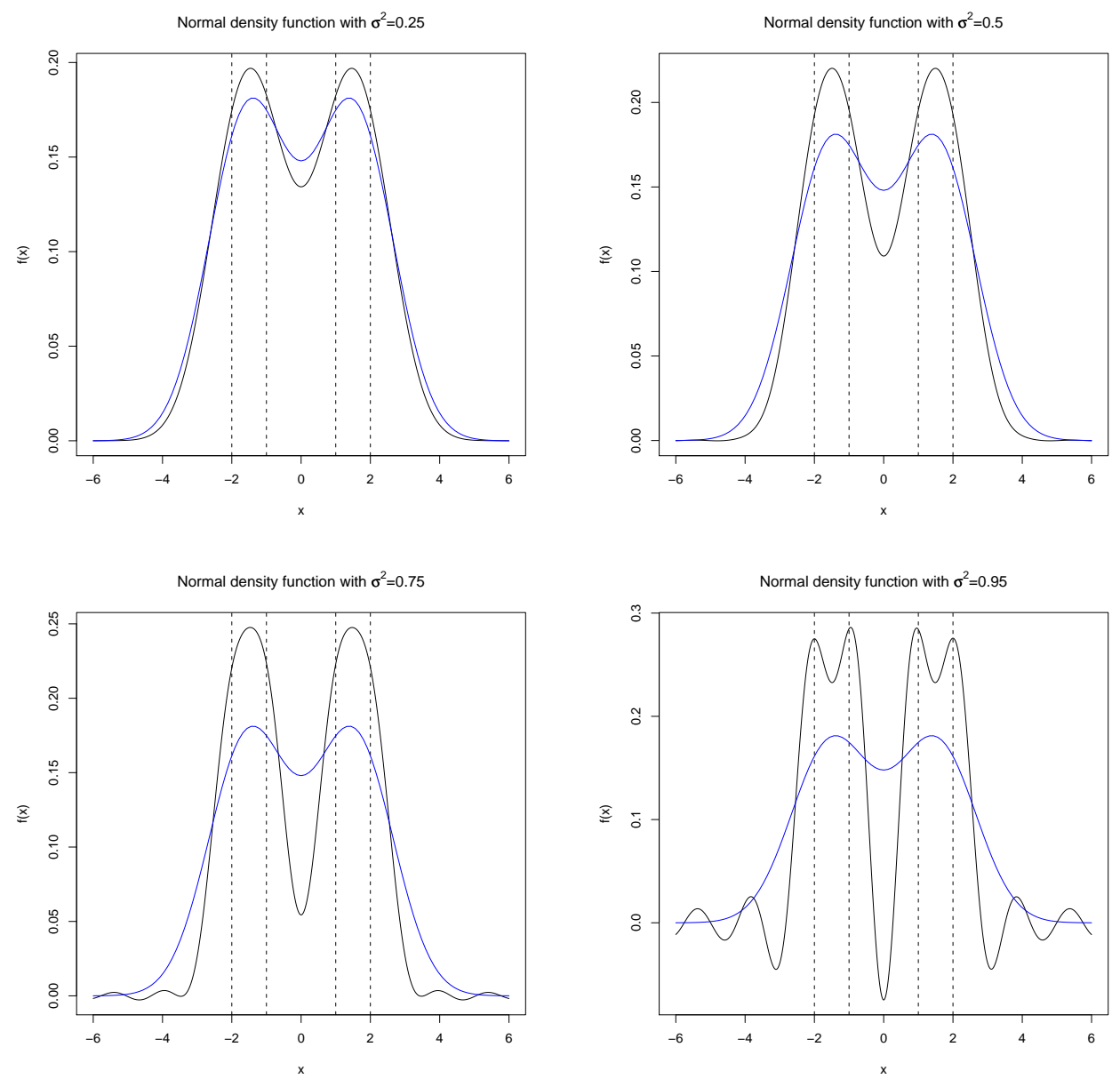

FIgURE 2.3: Mixture of normal densities with $\mu_{1}=-2, \mu_{2}=-1, \mu_{3}=1$, $\mu_{4}=2$ and $\sigma_{i}^{2}=1$. 

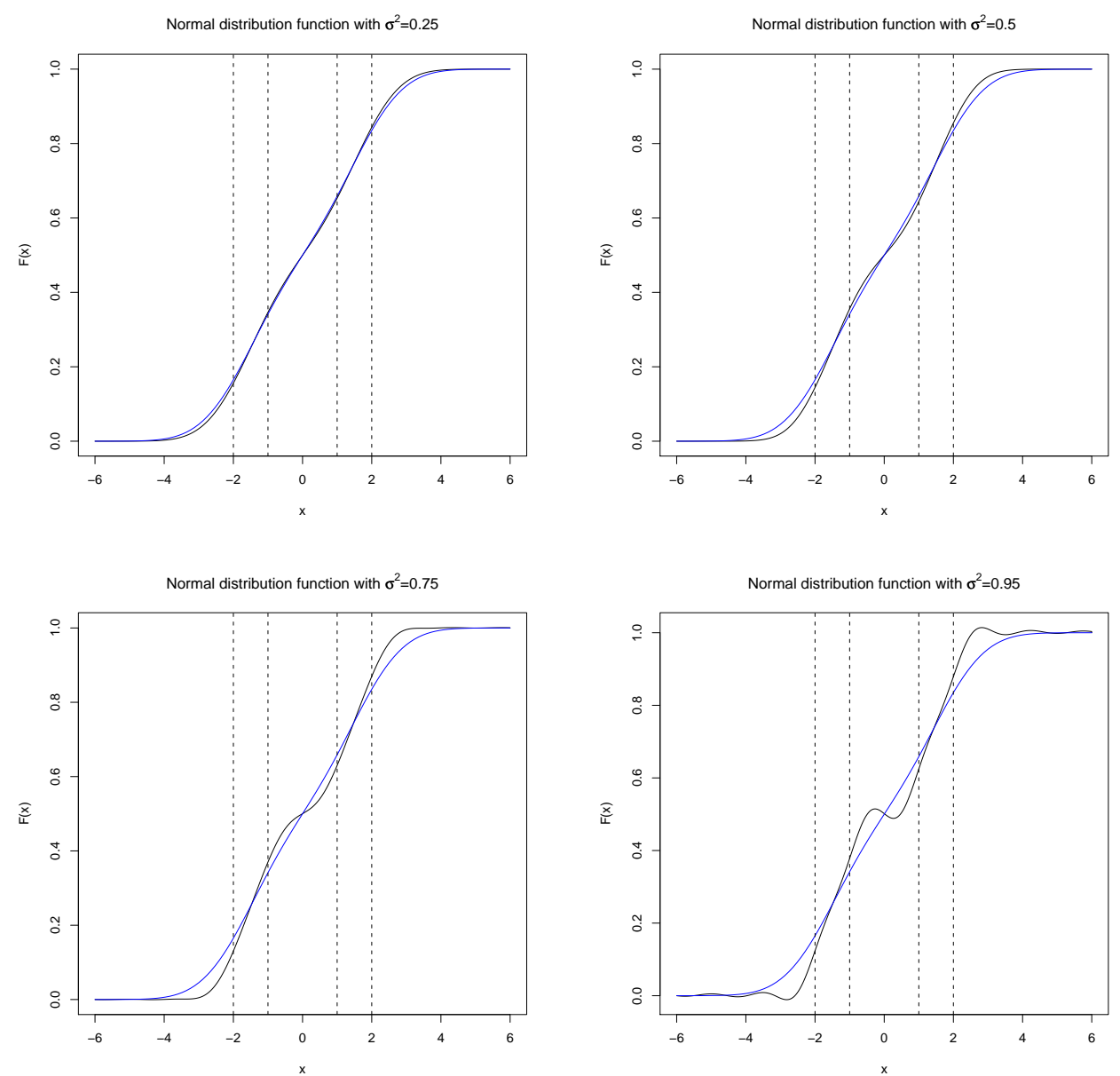

FiguRE 2.4: Mixture of normal distributions with $\mu_{1}=-2, \mu_{2}=-1, \mu_{3}=1$, $\mu_{4}=2$ and $\sigma_{i}^{2}=1$. 

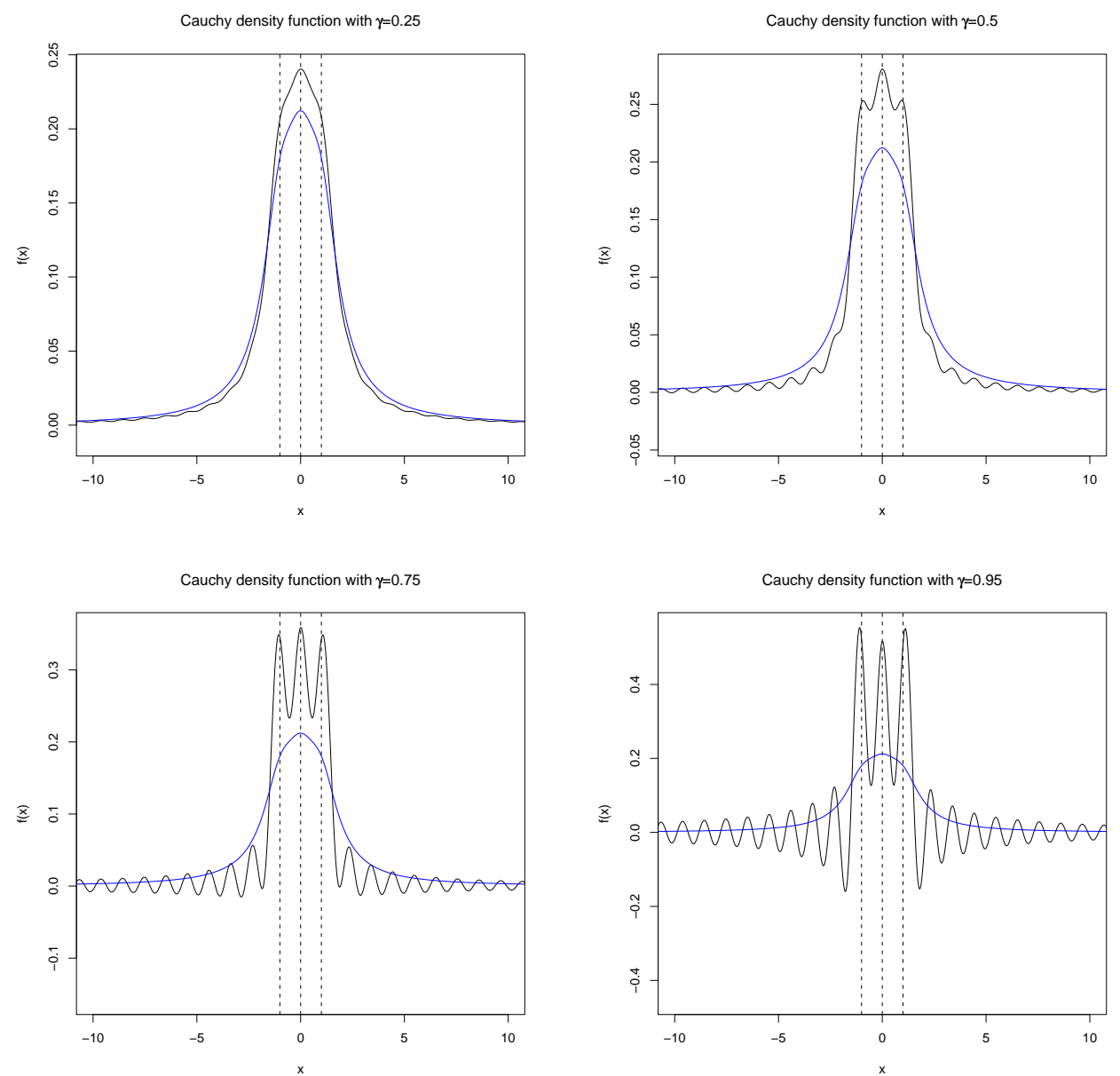

Figure 2.5: Mixture of Cauchy densities with scale parameter $=1$ for all components and shift paramters $\mu=(-1,0,1)$ 


\subsection{RESEARCH PROBLEM}

The main reference for this section is Hognas (1986), with other background references being Pflug (1991) and O'Brien (1972). Consider a sequence defined by

$$
X_{n+1}=f\left(X_{n}\right)+\xi_{n+1}
$$

where $\xi_{i}$ are independent and identically distributed with the standard normal distribution. If $f(x)=a x$ for $|a|<1$ constant, then $X_{\infty}$ has a limit distribution of $N\left(0, \frac{1}{1-a^{2}}\right)$. The problem posed by Goran Hognas is:

Given an arbitrary function $f(x)$ that lies between two linear functions $f_{1}(x)=a x$ and $f_{2}(x)=b x$, for all $x$, with $0<a<b<1$, does the limit distribution lie between the limit distributions of the sequences generated by $f_{1}(x)$ and $f_{2}(x)$ ?

The first function considered is

$$
f(x)=\frac{a+b}{2} x+\frac{b-a}{2} x \sin (x)
$$

The distribution function was constructed by first constructing the empirical distribution function from a generated data set of 100,000 observations of this process. The results for $b=0.75$ and $a=0.25$ are given in Figure 2.6. This answers the problem in the negative. Another choice of function is

$$
f(x)=\frac{a+b}{2} x+\frac{b-a}{2} x \sin (4 x)
$$


Empirical distribution function Sample Size $=100000$

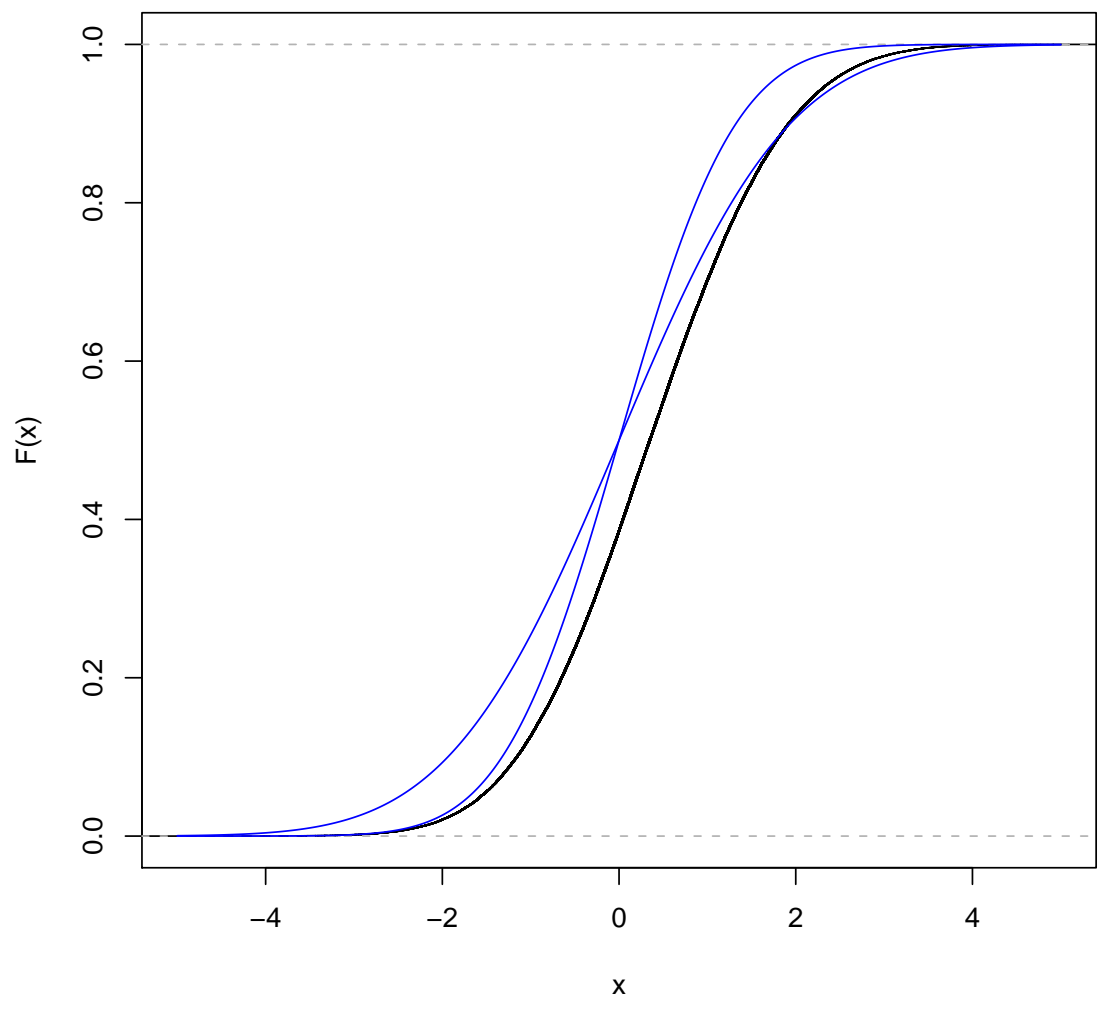

Figure 2.6: The blue lines indicate the limit distributions of the linear functions $a x$ and $b x$. The black line is the numerical estimate of the limit distribution using $f(x)=\frac{a+b}{2} x+\frac{b-a}{2} x \sin (x)$

with $a=0.75$ and $b=0.25$, which leads to the results in Figure 2.7. The final choice of function considered here is

$$
f(x)=\frac{a+b}{2} x+\frac{b-a}{2} x \cos (x)
$$

with $a=0.75$ and $b=0.25$, which leads to the results in Figure 2.8. These results show that general functions within the boundary of the linear 
Empirical distribution function Sample Size $=100000$

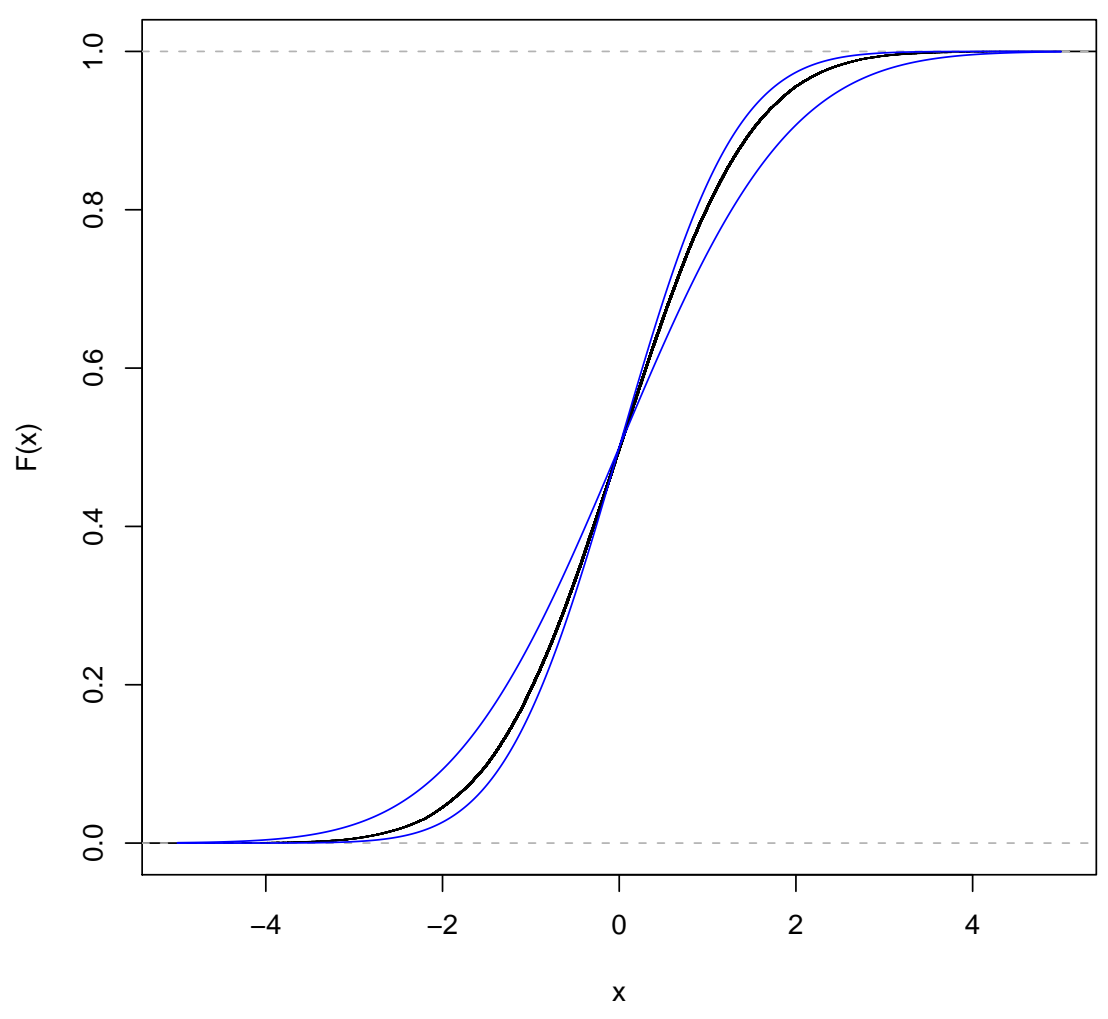

Figure 2.7: The blue lines indicate the limit distributions of the linear functions $a x$ and $b x$. The black line is the numerical estimate of the limit distribution using $f(x)=\frac{a+b}{2} x+\frac{b-a}{2} x \sin (4 x)$

functions $a x$ and $b x$ do not necessarily lead to a limit distribution between the limit distributions of the linear functions, however some non-linear functions could lead to limit functions between the limit distributions of the linear functions. Therefore, the actual behaviour of the function $f$ changes the limit distribution. The current conjecture is that for functions $f$, if we consider $f^{\prime}(x)=f(x)-\frac{a+b}{2} x$, if $f^{\prime}(x)$ is odd, then the limit distribution will lie within the limit distribution of the linear functions. 


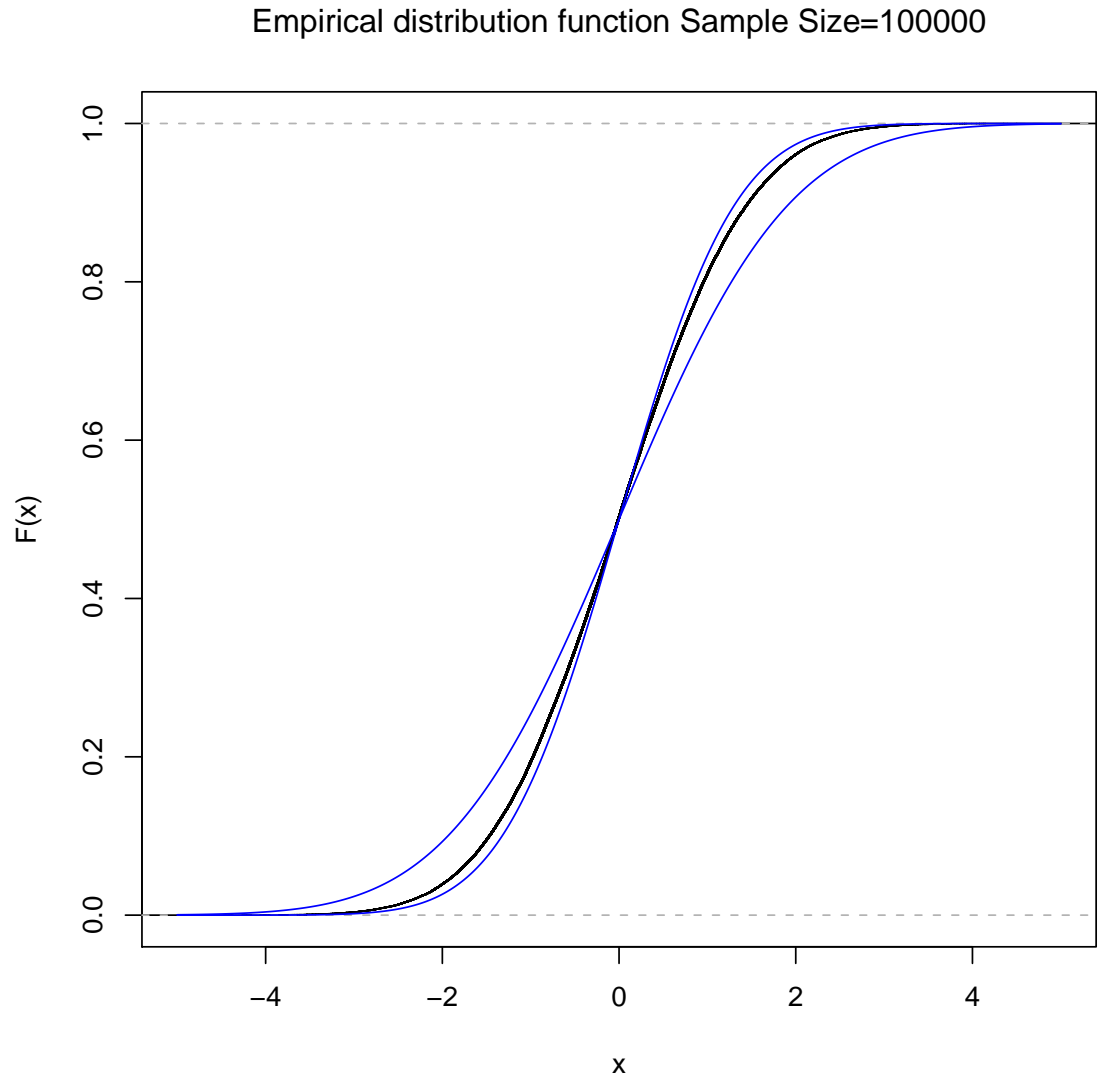

Figure 2.8: The blue lines indicate the limit distributions of the linear functions $a x$ and $b x$. The black line is the numerical estimate of the limit distribution using $f(x)=\frac{a+b}{2} x+\frac{b-a}{2} x \cos (x)$ 


\subsection{NEW APPROACH TO TESTING REGRESSION}

The reference for this section is Khmaladze \& Koul (2004). The aim of this section is to introduce a way of testing regression problems based on transformations of the errors process.

We are assuming that

$$
Y_{i}=m\left(X_{i} ; \theta\right)+\epsilon_{i}
$$

where $m\left(X_{i} ; \theta\right)$ is the regression function and $\epsilon_{i}$ are independent and identically distributed, with mean 0 and variance $\sigma^{2}$. If they do not have mean 0 , then we can add the bias of the error terms into the regression function and consider the new error terms which have mean 0 . If we have chosen $m\left(X_{i} ; \theta\right)$ correctly, then we can consider the partial sum process

$$
W_{n}(t)=\frac{1}{\sqrt{n}} \sum_{i \leq n t} \epsilon_{i} \quad 0 \leq t \leq 1
$$

which, as $n \rightarrow \infty$, has independent increments, mean 0 and variance $t \sigma^{2}$. By the central limit theorem the distribution for a given $t$ is Gaussian, so the limit is Brownian motion. Now consider estimating $\theta$ using Least-Squares. If 
$m\left(X_{i} ; \theta\right)$ is differentiable in $\theta$ then

$$
\begin{aligned}
\min _{\theta} \sum_{i=1}^{n} \epsilon_{i}^{2} & =\min _{\theta} \sum_{i=1}^{n}\left(Y_{i}-m\left(X_{i} ; \theta\right)\right)^{2} \\
& =\frac{\partial}{\partial \theta} \sum_{i=1}^{n}\left(Y_{i}-m\left(X_{i} ; \theta\right)\right)^{2} \\
& =\sum_{i=1}^{n} \frac{\partial}{\partial \theta}\left(Y_{i}-m\left(X_{i} ; \theta\right)\right)^{2} \\
& =-2 \sum_{i=1}^{n}\left(Y_{i}-m\left(X_{i} ; \theta\right)\right) m^{\prime}\left(X_{i} ; \theta\right)=0
\end{aligned}
$$

Letting $\hat{\theta}$ be the solution of (2.1), we can consider the partial sum process of the errors $\hat{\epsilon}_{i}$ under $\hat{\theta}$,

$$
\widehat{W}_{n}(t)=\frac{1}{\sqrt{n}} \sum_{i \leq n t}\left(Y_{i}-m\left(X_{i} ; \hat{\theta}\right)\right)=\frac{1}{\sqrt{n}} \sum_{i \leq n t} \hat{\epsilon}_{i} \quad 0 \leq t \leq 1
$$

We cannot apply the same logic to deduce that $\hat{W}_{n}(t)$ has a Brownian motion limit because the Least-Squares condition means that the errors are no longer independent. It follows from the Least-Squares condition that

$$
\int_{0}^{1} m^{\prime}\left(X_{n t} ; \hat{\theta}\right) d \hat{W}_{n}(t)=\frac{1}{\sqrt{n}} \sum_{k=1}^{n} m^{\prime}\left(X_{k} ; \hat{\theta}\right) \hat{\epsilon}_{k}=0
$$

As an example

$$
H_{0}: Y_{i}=a+\epsilon_{i}
$$

for constant $a$, then the Least-Squares estimator of $a$ is $\bar{Y}=\frac{1}{n} \sum_{i=1}^{n} Y_{i}$, and it follows from $(2.1)$ that $\widehat{W}_{n}(1)=0$, as

$$
\int_{0}^{1} m^{\prime}\left(X_{n t} ; \hat{\theta}\right) d \widehat{W}_{n}(t)=\frac{1}{\sqrt{n}} \sum_{k=1}^{n} \hat{\epsilon}_{k}=0 .
$$


This suggests that $\widehat{W}_{n}(t) \rightarrow \mathbb{V}$, where $\mathbb{V}$ is Brownian bridge, which is actually the case.

We want to modify $\hat{\epsilon}_{i}=Y_{i}-\bar{Y}$ in such a way that the partial sum process of the modified summands converges to Brownian motion. Consider the filtration $\mathcal{H}=\left\{\mathcal{H}_{i}\right\}$, where each $\mathcal{H}_{i}$ is the $\sigma$-algebra generated by $Y_{1}, Y_{2}, \ldots, Y_{i}, \bar{Y}$.

$$
E\left[Y_{i} \mid \mathcal{H}_{i-1}\right]=E\left[Y_{i} \mid \sum_{j=i}^{n} Y_{j}\right]
$$

This conditional expectation may be difficult to calculate, but we can consider a regression of $Y_{i}$ on $\sum_{j=i}^{n} Y_{j}$, with $\xi_{i}=Y_{i}-\gamma \sum_{j=i}^{n} Y_{j}$ for regression parameter $\gamma$. The Least-Squares estimator of $\gamma$ is

$$
\begin{gathered}
E\left[\left(Y_{i}-\gamma \sum_{j=i}^{n} Y_{j}\right) \sum_{j=i}^{n} Y_{j}\right]=0 \\
\Rightarrow E\left[Y_{i}^{2}-\gamma \sum_{j=i}^{n} Y_{j}^{2}\right]=0 \\
\Rightarrow \sigma^{2}=(n-i+1) \gamma \sigma^{2} \\
\Rightarrow \gamma=\frac{1}{n-i+1}
\end{gathered}
$$

Letting $\xi_{i}=\left(Y_{i}-\bar{Y}\right)-\frac{1}{n-i+1} \sum_{j=i}^{n}\left(Y_{j}-\bar{Y}\right)=Y_{i}-\frac{1}{n-i+1} \sum_{j=i}^{n} Y_{j}$, we find that for $i<j$, and assuming, without loss of generality, that $E\left[Y_{i}\right]=0$

$$
\begin{aligned}
E\left[\xi_{i} \xi_{j}\right]=E\left[Y_{i} Y_{j}\right. & -\frac{Y_{i}}{n-j+1} \sum_{l=j}^{n} Y_{l}-\frac{Y_{j}}{n-i+1} \sum_{k=i}^{n} Y_{k} \\
& \left.+\left(\frac{1}{n-i+1} \sum_{k=i}^{n} Y_{k}\right)\left(\frac{1}{n-j+1} \sum_{l=j}^{n} Y_{l}\right)\right]
\end{aligned}
$$


As $Y_{i}$ and $Y_{j}$ are independent with mean 0 it follows that

$$
E\left[\xi_{i} \xi_{j}\right]=0-0-\frac{\sigma^{2}}{n-i+1}+\frac{1}{n-i+1} \frac{1}{n-j+1}(n-j+1) \sigma^{2}=0
$$

We can also calculate the variance of $\xi_{i}$ as

$$
\begin{aligned}
E\left[\xi_{i}^{2}\right] & =E\left[Y_{i}^{2}-2 Y_{i} \frac{1}{n-i+1} \sum_{j=i}^{n} Y_{j}+\left(\frac{1}{n-i+1}\right)^{2}\left(\sum_{j=i}^{n} Y_{j}\right)^{2}\right] \\
& =\sigma^{2}-2 \frac{1}{n-i+1} \sigma^{2}+\left(\frac{1}{n-i+1}\right)^{2}(n-i+1) \sigma^{2} \\
& =\sigma^{2}\left(1-\frac{1}{n-i+1}\right)
\end{aligned}
$$

Therefore the partial sum process

$$
\widetilde{W}_{n}(t)=\frac{1}{\sqrt{n}} \sum_{i \leq n t} \xi_{i}
$$

is a sum of uncorrelated summands. It is also a martingale with respect to $\mathcal{H}$. Using martingale limit theory it is possible to show that $\widetilde{W}_{n}(t)$ converges to Brownian motion.

We can also reconstruct the original errors $\hat{\epsilon}_{i}$ from $\xi_{i}$. With $\xi_{i}=Y_{i}-$ $\frac{1}{n-i+1} \sum_{j=i}^{n} Y_{j}$, we find that $\xi_{1}=\hat{\epsilon}_{1}$,

$$
\hat{\epsilon}_{k}=\xi_{k}-\sum_{j=1}^{k-1}\left(\frac{1}{n-j} \xi_{j}\right) \quad k=2, \ldots, n-1
$$

and finally $\hat{\epsilon}_{n}=-\sum_{j=1}^{n-1} \hat{\epsilon}_{j}$. 
For the second example we will consider linear regression

$$
Y_{i}=a+b\left(X_{i}-\bar{X}\right)+\epsilon_{i}
$$

for $\epsilon_{i}$ independent and identically distributed. The Least-Squares estimators $a$ and $b$ must satisfy

$$
\begin{aligned}
& \frac{\partial}{\partial a} \sum_{i=1}^{n}\left(Y_{i}-a-b\left(X_{i}-\bar{X}\right)\right)^{2}=-\sum_{i=1}^{n}\left(Y_{i}-a-b\left(X_{i}-\bar{X}\right)\right)=0 \\
& \frac{\partial}{\partial b} \sum_{i=1}^{n}\left(Y_{i}-a-b\left(X_{i}-\bar{X}\right)\right)^{2}=-\sum_{i=1}^{n}\left(Y_{i}-a-b\left(X_{i}-\bar{X}\right)\right)\left(X_{i}-\bar{X}\right)=0
\end{aligned}
$$

From (2.2) it follows that

$$
a=\frac{1}{n} \sum_{i=1}^{n} Y_{i}-\frac{b}{n} \sum_{i=1}^{n}\left(X_{i}-\bar{X}\right)=\bar{Y}
$$

and from (2.3), substituting $a=\bar{Y}$

$$
\sum_{i=1}^{n}\left(X_{i}-\bar{X}\right)\left(Y_{i}-\bar{Y}\right)=b \sum_{i=1}^{n}\left(X_{i}-\bar{X}\right)^{2} \Rightarrow b=\frac{\sum_{i=1}^{n}\left(X_{i}-\bar{X}\right)\left(Y_{i}-\bar{Y}\right)}{\sum_{i=1}^{n}\left(X_{i}-\bar{X}\right)^{2}}
$$

Therefore

$$
Y_{i}=\bar{Y}+\frac{\sum_{i=1}^{n}\left(X_{i}-\bar{X}\right)\left(Y_{i}-\bar{Y}\right)}{\sum_{i=1}^{n}\left(X_{i}-\bar{X}\right)^{2}}\left(X_{i}-\bar{X}\right)+\hat{\epsilon}_{i}
$$


In this case, the limit of the partial sum process is subject to the conditions

$$
\begin{aligned}
\int_{0}^{1} d \widehat{W}_{n}(t) & =\frac{1}{\sqrt{n}} \sum_{k=1}^{n} \hat{\epsilon}_{k}=0 \\
\int_{0}^{1}\left(X_{t}-\bar{X}\right) d \widehat{W}_{n}(t) & =\frac{1}{\sqrt{n}} \sum_{k=1}^{n}\left(X_{k}-\bar{X}\right) \hat{\epsilon}_{k}=0
\end{aligned}
$$

so the limit is Brownian bridge subject to the extra condition (2.5). We want to modify the partial sum process so that the modified summands $\xi_{i}$ are uncorrelated. As we have added a new variable to the regression function, we have a new filtration $\mathcal{H}=\left\{\mathcal{H}_{i}\right\}$, where each $\mathcal{H}_{i}$ is the $\sigma$-algebra generated by $Y_{1}, Y_{2}, \ldots, Y_{i}, \bar{Y}, X_{1}, X_{2}, \ldots, X_{n}, \sum_{i=1}^{n}\left(X_{i}-\bar{X}\right)\left(Y_{i}-\bar{Y}\right)$. We want to again modify the partial sum process so that the modified partial sum process converges to Brownian motion.

$$
E\left[\epsilon_{i} \mid \mathcal{H}_{i-1}\right]=E\left[\epsilon_{i} \mid \sum_{j=i}^{n} \epsilon_{j}, \sum_{j=i}^{n}\left(X_{j}-\bar{X}\right) \epsilon_{j}\right]
$$

If we know $\sum_{j=i}^{n}\left(X_{j}-\bar{X}\right) \epsilon_{j}$ and $\sum_{j=i}^{n} \epsilon_{j}$, then we also know $\sum_{j=i}^{n} X_{j} \epsilon_{j}$, from which we can calculate $\sum_{j=i}^{n}\left(X_{j}-\bar{X}_{i, n}\right) \epsilon_{j}$, where $\bar{X}_{i, n}=\frac{1}{n-i+1} \sum_{j=i}^{n} X_{j}$ are partial averages. It can be shown that $\sum_{j=i}^{n} \epsilon_{j}$ and $\sum_{j=i}^{n}\left(X_{j}-\bar{X}_{i, n}\right) \epsilon_{j}$ are uncorrelated.

$$
=E\left[\epsilon_{i} \mid \sum_{j=i}^{n} \epsilon_{j}, \sum_{j=i}^{n}\left(X_{j}-\bar{X}_{i, n}\right) \epsilon_{j}\right]
$$


We can consider a regression of $\epsilon_{i}$ on $\sum_{j=i}^{n} \epsilon_{j}$ and $\sum_{j=i}^{n}\left(X_{j}-\bar{X}_{i, n}\right) \epsilon_{j}$, where

$$
\xi_{i}=\epsilon_{i}-\alpha_{i} \sum_{j=i}^{n} \epsilon_{j}-\beta_{i} \sum_{j=i}^{n}\left(X_{j}-\bar{X}_{i, n}\right) \epsilon_{j}
$$

so using Least-Squares

$$
\begin{aligned}
\frac{\partial}{\partial \alpha_{i}} E\left[\sum_{k=1}^{n} \xi_{k}^{2}\right]=0 & =E\left[\xi_{i}\left(\sum_{j=i}^{n} \epsilon_{j}\right)\right]=\sigma^{2}-\alpha_{i} \sigma^{2}(n-i+1) \\
\frac{\partial}{\partial \beta_{i}} E\left[\sum_{k=1}^{n} \xi_{k}^{2}\right]=0 & =E\left[\xi_{i}\left(\sum_{j=i}^{n}\left(X_{j}-\bar{X}\right) \epsilon_{j}\right)\right] \\
& =\sigma^{2}\left(X_{i}-\bar{X}_{i, n}\right)-\beta_{i} \sum_{j=i}^{n}\left(X_{j}-\bar{X}_{i, n}\right)^{2}
\end{aligned}
$$

From (2.6) it follows

$$
\alpha_{i}=\frac{1}{n-i+1} \quad \beta_{i}=\frac{X_{i}-\bar{X}_{i, n}}{\sum_{j=i}^{n}\left(X_{j}-\bar{X}_{i, n}\right)^{2}}
$$

Therefore

$$
\xi_{i}=\epsilon_{i}-\frac{1}{n-i+1} \sum_{j=i}^{n} \epsilon_{j}-\frac{X_{i}-\bar{X}_{i, n}}{\sum_{j=i}^{n}\left(X_{j}-\bar{X}_{i, n}\right)^{2}} \sum_{j=i}^{n}\left(X_{j}-\bar{X}_{i, n}\right) \epsilon_{j}
$$

We also note that $\epsilon_{i}-c$ can be substituted in place of $\epsilon_{i}$ in the above equation without changing $\xi_{i}$, so it follows that if

$$
\xi_{i}^{\prime}=\hat{\epsilon}_{i}-\frac{1}{n-i+1} \sum_{j=i}^{n} \hat{\epsilon}_{j}-\frac{X_{i}-\bar{X}_{i, n}}{\sum_{j=i}^{n}\left(X_{j}-\bar{X}_{i, n}\right)^{2}} \sum_{j=i}^{n}\left(X_{j}-\bar{X}_{i, n}\right) \hat{\epsilon}_{j}
$$


and that

$$
\widetilde{W}_{n}(t)=\frac{1}{\sqrt{n}} \sum_{i \leq n t} \xi_{i}^{\prime}
$$

is Brownian motion.

\subsection{RANKS}

\subsubsection{INTRODUCTION TO RANKS}

For this section assume that $X_{i} \in \mathbb{R}$. Define

$$
\min _{1 \leq i \leq n} X_{i}=X_{1: n} \leq X_{2: n} \leq \ldots \leq X_{n: n}=\max _{1 \leq i \leq n} X_{i}
$$

as the order statistics for $i=1, \ldots, n$.

Definition 2.4.1: Let $X_{1}, X_{2}, \ldots, X_{n}$ be a sequence of random variables. The rank of $X_{i}$, denoted $R_{i}$, is $R_{i}=\sum_{i=1}^{n} \mathbb{I}_{\left\{X_{j} \leq X_{i}\right\}}$

It is also convenient to assume that the underlying distribution function $F$ is continuous on $\mathbb{R}$, as then the sample elements are almost surely different.

Result 2.4.2: For continuous $F$ we have the following

$$
F_{n}\left(X_{i}\right)=\frac{1}{n} R_{i n} ; \quad F_{n}\left(X_{i: n}\right)=\frac{i}{n} ; \quad F_{n}^{-1}\left(\frac{i}{n}\right)=X_{i: n}
$$

Proof: The result follows immediately from the definition. 
For the next theorem we need to define

$$
\mathbb{O}_{n}=\left\{\left\langle t_{1}, \ldots, t_{n}\right\rangle \in \mathbb{R}^{n}: t_{1} \leq \ldots \leq t_{n}\right\}
$$

and $\mathcal{R}_{n}$ as the set of permutations of $\langle 1, \ldots, n\rangle$

Theorem 2.4.3: For continuous $F, O \subset \mathbb{O}$ and $\left(r_{1}, \ldots, r_{n}\right) \in \mathcal{R}_{n}$ we have

1. $\mathbb{P}\left\{\left\langle X_{1: n}, \ldots, X_{n: n}\right\rangle \in O\right\}=n ! \mathbb{P}\left\{\left\langle X_{1}, \ldots, X_{n}\right\rangle \in O\right\}$

2. $\mathbb{P}\left\{\left\langle R_{1 n}, \ldots, R_{n n}\right\rangle=\left\langle r_{1}, \ldots, r_{n}\right\rangle\right\}=\frac{1}{n !}$

3. $\left\langle X_{1: n}, \ldots, X_{n: n}\right\rangle$ and $\left\langle R_{1 n}, \ldots, R_{n n}\right\rangle$ are independent.

Proof: Let $\rho=\left\langle\rho_{1}, \ldots, \rho_{n}\right\rangle$ be the inverse permutation of $\left\langle r_{1}, \ldots, r_{n}\right\rangle$. Then it is clear that

$$
\begin{aligned}
& \mathbb{P}\left\{\left\langle R_{1 n}, \ldots, R_{n n}\right\rangle=\left\langle r_{1}, \ldots, r_{n}\right\rangle,\left\langle X_{1: n}, \ldots, X_{n: n}\right\rangle \in O\right\} \\
& =\mathbb{P}\left\{\left\langle X_{\rho_{1}}, \ldots, X_{\rho_{n}}\right\rangle\right\} \\
& =\mathbb{P}\left\{\left\langle X_{1}, \ldots, X_{n}\right\rangle \in O\right\}
\end{aligned}
$$


Letting $O=\mathbb{O}$ we get

$$
\begin{aligned}
& \mathbb{P}\left\{\left\langle R_{1 n}, \ldots, R_{n n}\right\rangle=\left\langle r_{1}, \ldots, r_{n}\right\rangle\right\} \\
& =\mathbb{P}\left\{\left\langle R_{1 n}, \ldots, R_{n n}\right\rangle=\left\langle r_{1}, \ldots, r_{n}\right\rangle,\left\langle X_{1: n}, \ldots, X_{n: n}\right\rangle \in \mathbb{O}\right\} \\
& =\mathbb{P}\left\{\left\langle X_{1}, \ldots, X_{n}\right\rangle \in \mathbb{O}\right\} \\
& =\mathbb{P}\left\{X_{1} \leq \ldots \leq X_{n}\right\} \\
& =\frac{1}{n !}
\end{aligned}
$$

By summing over the possible permutations of the ranks it is clear that

$$
\begin{aligned}
& \mathbb{P}\left\{\left\langle X_{1: n}, \ldots, X_{n: n}\right\rangle \in O\right\} \\
& =n ! \mathbb{P}\left\{\left\langle X_{1}, \ldots, X_{n}\right\rangle \in O\right\}
\end{aligned}
$$

This leads to

$$
\begin{aligned}
& \mathbb{P}\left\{\left\langle R_{1 n}, \ldots, R_{n n}\right\rangle=\left\langle r_{1}, \ldots, r_{n}\right\rangle,\left\langle X_{1: n}, \ldots, X_{n: n}\right\rangle \in O\right\} \\
& =\mathbb{P}\left\{\left\langle X_{1}, \ldots, X_{n}\right\rangle \in O\right\} \\
& =\frac{1}{n !} \mathbb{P}\left\{\left\langle X_{1: n}, \ldots, X_{n: n}\right\rangle \in O\right\} \\
& =\mathbb{P}\left\{\left\langle R_{1 n}, \ldots, R_{n n}\right\rangle=\left\langle r_{1}, \ldots, r_{n}\right\rangle\right\} \mathbb{P}\left\{\left\langle X_{1: n}, \ldots, X_{n: n}\right\rangle \in O\right\}
\end{aligned}
$$

This leads us to consider the marginal distributions. 


\section{TheOREM 2.4.4:}

$$
\mathbb{P}\left\{R_{\text {in }}=j\right\}=\frac{1}{n} ; \quad \forall j \in\{1, \ldots, n\}
$$

Proof: This is clear by considering the number of possible values of $R_{\text {in }}$ $(1, \ldots, n)$ and noticing that because the sample is independent and identically distributed that all values have equal probability.

\section{TheOREM 2.4.5:}

$$
\begin{aligned}
\mathbb{P}\left\{X_{i: n} \leq t\right\} & =\frac{n !}{(i-1) !(n-i) !} \int_{0}^{F(t)} s^{i-1}(1-s)^{n-i} d s \\
& =\sum_{k=i}^{n}\left(\begin{array}{l}
n \\
k
\end{array}\right)[F(t)]^{k}[1-F(t)]^{n-k}
\end{aligned}
$$

Proof: The first expression assumes that there is a derivative $f$ of $F$ on $\mathbb{R}$. Using the multinomial distribution, it is seen that

$$
\begin{aligned}
& \mathbb{P}\left\{t<X_{i: n} \leq t+\Delta\right\} \\
& =\frac{n !}{(i-1) ! 1 !(n-i) !}[F(t)]^{i-1}[F(t+\Delta)-F(t)][1-F(t)]^{n-i}+O\left(\Delta^{2}\right)
\end{aligned}
$$

Dividing by $\Delta$ and taking the limit for $\Delta \downarrow 0$ we find the density $g$ for the distribution of $X_{i: n}$ as

$$
g(t)=\frac{n !}{(i-1) !(n-i) !}[F(t)]^{i-1}[1-F(t)]^{n-i} f(t)
$$

Taking the integral of $g(t)$ from 0 to $s$ and making a substitution $s=F(t)$ 
gives

$$
\begin{aligned}
\mathbb{P}\left\{X_{i: n} \leq t\right\} & =\frac{n !}{(i-1) !(n-i) !} \int_{0}^{F(t)} s^{i-1}(1-s)^{n-i} d s \\
& =\sum_{k=i}^{n}\left(\begin{array}{l}
n \\
k
\end{array}\right)[F(t)]^{k}[1-F(t)]^{n-k}
\end{aligned}
$$

To derive the second expression it is useful to note that

$$
\left\{X_{i: n} \leq t\right\} \Leftrightarrow\left\{F_{n}(t) \geq t\right\}
$$

By conditioning on the number of $X_{i} \leq t$ and summing over the events the second expression immediately follows.

\subsubsection{APPLICATIONS}

\section{ExAmple: (Two Sample Problem)}

Suppose that $X_{1}, \ldots, X_{n_{1}}$ is a random sample of size $n_{1}$ from a continuous distribution function $F_{1}$ on $\mathbb{R}$ and that $X_{n_{1}+1}, \ldots, X_{n_{1}+n_{2}}$ is a random sample of size $n_{2}$ from a continuous distribution function $F_{2}$ on $\mathbb{R}$. The two sample problem tests the null hypothesis of $F_{1}=F_{2}$ versus the alternative of inequality. Let $n=n_{1}+n_{2}$ and $R_{1 n}, \ldots, R_{n n}$ be the ranks of the pooled sample elements. Then a good test statistic would be

$$
\sum_{i=1}^{n_{1}} R_{\text {in }} \text { or } \quad \sum_{i=1}^{n_{1}} \frac{R_{\text {in }}}{n+1}
$$


which would be rejected for small or large values. Assume from now that the null hypothesis is true and that $F$ is the common distribution function.

Theorem 2.4.6: Suppose for each $n$ the real numbers $c_{1 n}, \ldots, c_{n n}$ satisfy

$$
\sum_{i=1}^{n} c_{i n}=0, \quad \sum_{i=1}^{n} c_{i n}^{2}=1, \quad \max _{1 \leq i \leq n} c_{i n} \rightarrow 0
$$

Furthermore let

$$
W_{n}=(12)^{\frac{1}{2}} \sum_{i=1}^{n} c_{i n} \frac{R_{i n}}{n+1}, \quad S_{n}=(12)^{\frac{1}{2}} \sum_{i=1}^{n} c_{i n} \xi_{i}
$$

where $\xi_{i}=F\left(X_{i}\right)$ are independent with distribution $\mathcal{U}(0,1)$ under the null hypothesis. Then

$$
\mathbb{P}\left\{W_{n} \leq x\right\} \stackrel{n \rightarrow \infty}{\longrightarrow} \Phi(x) \quad \forall x \in \mathbb{R}
$$

Proof: The first part is to show $S_{n}$ converges to the standard normal distribution.

$$
\begin{aligned}
E\left[S_{n}\right] & =(12)^{\frac{1}{2}}\left(\sum_{i=1}^{n} c_{i n}\right) E\left[\xi_{i}\right]=0 \\
\operatorname{Var}\left(S_{n}\right) & =12\left(\sum_{i=1}^{n} c_{i n}^{2}\right) \operatorname{Var}\left(\xi_{i}\right)=12(1)\left(\frac{1}{12}\right)=1
\end{aligned}
$$


Therefore $S_{n}$ converges to the standard normal distribution using the central limit theorem. The next stage is to show that $W_{n} \rightarrow S_{n}$ in $\mathcal{L}^{2}$, which implies convergence in probability, which implies convergence in distribution. Let

$$
\left(V_{1}, \ldots, V_{n}\right)=\left(\frac{R_{1 n}}{n+1}-\xi_{1}, \ldots, \frac{R_{n n}}{n+1}-\xi_{n}\right)
$$

which has exchangeable components. This implies that $\mathcal{L}\left(V_{i}\right)=\mathcal{L}\left(V_{1}\right)$ and $\mathcal{L}\left(V_{i} V_{j}\right)=\mathcal{L}\left(V_{1} V_{2}\right) ; \forall i \neq j$.

$$
\begin{aligned}
\frac{1}{12} E\left[W_{n}-S_{n}\right]^{2} & =E\left[\sum_{i=1}^{n} c_{i n} V_{i}\right]^{2} \\
& =\sum_{i \neq j} c_{i n} c_{j n} E\left[V_{i} V_{j}\right]+\sum_{i=1}^{n} c_{i n}^{2} E\left[V_{i}^{2}\right] \\
& =\left(\sum_{i=1}^{n} c_{i n}\right)^{2} E\left[V_{1} V_{2}\right]+\left(\sum_{i=1}^{n} c_{i n}^{2}\right)\left(E\left[V_{1}^{2}\right]-E\left[V_{1} V_{2}\right]\right) \\
& \leq 2 E\left[V_{1}^{2}\right]
\end{aligned}
$$

All that remains is to show that $E\left[V_{1}^{2}\right] \rightarrow 0$.

$$
\begin{aligned}
E\left[V_{1}^{2}\right] & =E\left[E\left[\left(\frac{R_{1 n}}{n+1}-\xi_{i}\right)^{2} \mid R_{1 n}=i\right]\right] \\
& =\sum_{i=1}^{n} E\left[\left(\frac{R_{1 n}}{n+1}-\xi_{i}\right)^{2} \mid R_{1 n}=i\right] \mathbb{P}\left\{R_{1 n}=i\right\} \\
& =\frac{1}{n} \sum_{i=1}^{n} E\left[\left(\frac{i}{n+1}-\xi_{i: n}\right)^{2}\right]
\end{aligned}
$$


So using Theorem 2.4.4 and Theorem 2.4.5 on $\xi_{i}$, we see the distribution function of $\xi_{i}$ for $0 \leq t \leq 1$ is

$$
\begin{aligned}
\mathbb{P}\left\{\xi_{i: n} \leq t\right\} & =\frac{n !}{(i-1) !(n-i) !} \int_{0}^{t} s^{i-1}(1-s)^{n-i} d F(s) \\
& =\frac{n !}{(i-1) !(n-i) !} \int_{0}^{t} s^{i-1}(1-s)^{n-i} d s
\end{aligned}
$$

Which gives the first and second moments as

$$
\begin{aligned}
E\left[\xi_{i: n}\right] & =\frac{n !}{(i-1) !(n-i) !} \int_{0}^{1} s^{i}(1-s)^{n-i} d s \\
& =\frac{n !}{(i-1) !(n-i) !} \frac{(i) !(n-i) !}{(n+1) !}=\frac{i}{n+1} \\
E\left[\xi_{i: n}^{2}\right] & =\frac{n !}{(i-1) !(n-i) !} \int_{0}^{1} s^{i+1}(1-s)^{n-i} d s \\
& =\frac{n !}{(i-1) !(n-i) !} \frac{(i+1) !(n-i) !}{(n+2) !}=\frac{(i+1) i}{(n+2)(n+1)}
\end{aligned}
$$

Therefore

$$
\begin{aligned}
E\left[\left(\frac{i}{n+1}-\xi_{i: n}\right)^{2}\right] & =E\left[\xi_{i: n}^{2}\right]-\left(E\left[\xi_{i: n}\right]\right)^{2} \\
& =\frac{(i+1) i}{(n+2)(n+1)}-\left(\frac{i}{n+1}\right)^{2}=\frac{i(n-i+1)}{(n+2)(n+1)^{2}}
\end{aligned}
$$

This gives an upper bound for $\frac{1}{12} E\left[W_{n}-S_{n}\right]^{2}$ as

$$
\begin{aligned}
\frac{1}{12} E\left[W_{n}-S_{n}\right]^{2} & \leq 2 E\left[V_{1}^{2}\right] \\
& =2 \sum_{i=1}^{n} \frac{i(n-i+1)}{n(n+2)(n+1)^{2}} \downarrow 0 \quad \text { as } n \rightarrow \infty
\end{aligned}
$$


Corollary 2.4.7: (Wilcoxin's two-sample test) For the two sample problem

$$
\mathbb{P}\left\{\left(\frac{12 n}{n_{1} n_{2}}\right)^{\frac{1}{2}} \sum_{i=1}^{n_{1}}\left(\frac{R_{\text {in }}}{n+1}-\frac{1}{2}\right) \leq x\right\} \rightarrow \Phi(x) \quad \text { as } n_{1} \wedge n_{2} \rightarrow \infty ; \forall x \in \mathbb{R}
$$

Proof: Let

$$
c_{i n}= \begin{cases}\left(\frac{n_{2}}{n n_{1}}\right)^{\frac{1}{2}} & \text { for } i=1, \ldots, n_{1} \\ -\left(\frac{n_{1}}{n n_{2}}\right)^{\frac{1}{2}} & \text { for } i=n_{1}+1, \ldots, n_{1}+n_{2}\end{cases}
$$

Then we only need to show it satisfies the conditions for THEOREM 2.4.6.

1. $\sum_{i=1}^{n} c_{i n}=0$

$$
\begin{aligned}
\sum_{i=1}^{n} c_{i n} & =\sum_{i=1}^{n_{1}}\left(\frac{n_{2}}{n n_{1}}\right)^{\frac{1}{2}}+\sum_{i=1}^{n_{2}}\left(-\frac{n_{1}}{n n_{2}}\right)^{\frac{1}{2}} \\
& =n_{1}\left(\frac{n_{2}}{n n_{1}}\right)^{\frac{1}{2}}+n_{2}\left(-\frac{n_{1}}{n n_{2}}\right)^{\frac{1}{2}} \\
& =\left(\frac{n_{1} n_{2}}{n}\right)^{\frac{1}{2}}+\left(-\frac{n_{1} n_{2}}{n}\right)^{\frac{1}{2}}=0
\end{aligned}
$$


2. $\sum_{i=1}^{n} c_{i n}^{2}=0$

$$
\begin{aligned}
\sum_{i=1}^{n} c_{i n}^{2} & =\sum_{i=1}^{n_{1}}\left(\frac{n_{2}}{n n_{1}}\right)+\sum_{i=1}^{n_{2}}\left(\frac{n_{1}}{n n_{2}}\right) \\
& =\left(\frac{n_{2}}{n}\right)+\left(\frac{n_{1}}{n}\right)=1
\end{aligned}
$$

3. $\max _{1 \leq i \leq n} c_{i n} \rightarrow 0$

For $i=1, \ldots, n_{1}$

$$
\left|c_{i n}\right| \leq \frac{1}{\left(n_{1}\right)^{\frac{1}{2}}}
$$

and for $i=n_{1}+1, \ldots, n_{1}+n_{2}$

$$
\left|c_{i n}\right| \leq \frac{1}{\left(n_{2}\right)^{\frac{1}{2}}}
$$

Both of these $\rightarrow 0$ as $n_{1} \wedge n_{2} \rightarrow \infty$, concluding the proof.

\subsubsection{SEQUENTIAL RANKS}

The reference for this subsection is KhmaLADzE (2010). We are assumig that the underlying distribution of the $X_{i}$ is continuous for this subsection.

Definition 2.4.8: Let $X_{1}, X_{2}, \ldots, X_{n}$ be a sequence of random variables. 
The sequential rank of $X_{i}$, denoted $S_{i}$, is

$$
S_{i}=\sum_{j=1}^{i} \mathbb{I}_{\left\{X_{j} \leq X_{i}\right\}}
$$

The sequential rank is the rank of $X_{i}$ among only the first $i$ observations. At this stage it is useful to see a comparison of the properties of ranks and sequential ranks.

Proposition 2.4.9: For sequential ranks $S_{i} \in\{1, \ldots, i\}$ with $S_{1}=1$ and $S_{n}=R_{n}$, whereas for ranks $R_{i} \in\{1, \ldots, n\}$

Proposition 2.4.10: For $i \neq j, \mathbb{P}\left\{S_{i}=k \mid S_{j}=l\right\}=\mathbb{P}\left\{S_{i}=k\right\}$, so the sequential ranks are independent. For ranks $\mathbb{P}\left\{R_{i}=k \mid R_{j}=k\right\}=0$ where $\mathbb{P}\left\{R_{i}=k\right\} \neq 0$ for all $k$, so ranks are not independent. Further, $\sum_{i=1}^{n} \phi\left(S_{i}\right)$ is the sum of independent random variables and is non-degenerate random variable, whereas $\sum_{i=1}^{n} \phi\left(R_{i}\right)=\sum_{i=1}^{n} \phi(i)$ is constant.

Theorem 2.4.11: The relationship between the vector of ranks and the vector of sequential ranks is one-to-one.

Proof: Fix a sample of size $n$. To get the sequential ranks from the ranks, we consider the sequential ranks of the sequence of random variables $R_{1}, \ldots, R_{n}$, so

$$
S_{i}=\sum_{j=1}^{i} \mathbb{I}_{\left\{R_{j} \leq R_{i}\right\}}=\sum_{j=1}^{i} \mathbb{I}_{\left\{X_{j} \leq X_{i}\right\}}
$$

where $X_{j} \leq X_{i}$ if and only if $R_{j} \leq R_{i}$. 
To get the ranks from the sequential ranks we start with the known rank $R_{n}=S_{n}$. Now consider $S_{n-1}$. If $S_{n} \leq S_{n-1}$, then $X_{n}<X_{n-1}$, so $R_{n-1}=S_{n-1}+1$. If $S_{n}>S_{n-1}$, then $X_{n}>X_{n-1}$, so $X_{n}$ does not affect the rank of $X_{n-1}$, so $R_{n-1}=S_{n-1}$. It follows that $R_{n-1}=S_{n-1}+\mathbb{I}_{\left\{S_{n-1} \geq S_{n}\right\}}$. Similar reasoning leads to

$$
R_{i}=S_{i}+\sum_{j=i+1}^{n} \mathbb{I}_{\left\{S_{i} \geq S_{j}\right\}} \quad i=1,2, \ldots, n-1
$$

where $R_{n}=S_{n}$.

When applying ranks in testing problems, such as the linear rank statistics and goodness of fit statistics, the statistics are usually based on the empirical field

$$
z_{R}(t, u)=\sum_{i=1}^{n t}\left[\mathbb{I}_{\left\{R_{i n} \leq u(n+1)\right\}}-\frac{[n u]}{n+1}\right], \quad(t, u) \in[0,1]^{2}
$$

The linear rank statistics are linear functionals from the empirical field

$$
\psi\left(\mathbf{R}_{n}\right)=\int \psi(t, u) z_{R}(d t, d u)=\sum_{i=1}^{n}\left(\psi\left(\frac{i}{n}, \frac{R_{i n}}{n+1}\right)-E\left[\psi\left(\frac{i}{n}, \frac{R_{i n}}{n+1}\right)\right]\right)
$$

where $\mathbf{R}_{n}$ is a vector of ranks of size $n$.

Theorem 2.4.12: If, under the null hypothesis, the sample is independent and identically distributed, while under the alternative hypothesis the distribution $A_{i}$ of each $X_{i}$ is such that

$$
\frac{d A_{i}(x)}{d F(x)}=1+\frac{1}{\sqrt{n}} a\left(\frac{i}{n}, F(x)\right)+\text { smaller terms }, \quad n \rightarrow \infty
$$


where $\int_{0}^{1} a(t, F(x)) d t=0$, then the linear rank statistic, with $\psi=a$,

$$
a\left(\mathbf{R}_{n}\right)=\sum_{i=1}^{n} a\left(\frac{i}{n}, \frac{R_{\text {in }}}{n+1}\right)
$$

is asymptotically optimal against this alternative.

Proof: See Hajek et AL. (1999), among others.

Returning to sequential ranks, we would expect that we could use sequential ranks for testing instead of ranks, and still have asymptotic optimality. We can construct an empirical field based on sequential ranks

$$
z_{S}(t, u)=\sum_{i=1}^{n t}\left[\mathbb{I}_{\left\{S_{i} \leq u(i+1)\right\}}-\frac{[i u]}{i+1}\right], \quad(t, u) \in[0,1]^{2}
$$

and sequential linear rank statistics based on the empirical field

$$
\phi\left(\mathbf{S}_{n}\right)=\int \phi(t, u) z_{S}(d t, d u)=\sum_{i=1}^{n}\left(\psi\left(\frac{i}{n}, \frac{S_{i}}{i+1}\right)-E\left[\psi\left(\frac{i}{n}, \frac{S_{i}}{i+1}\right)\right]\right) .
$$

However, $a\left(\mathbf{S}_{n}\right)=\sum_{i=1}^{n} a\left(\frac{i}{n}, \underline{S_{i}} i+1\right)$ is not optimal against (2.8).

Theorem 2.4.13: The empirical fields $z_{R}$ and $z_{S}$ are asymptotically linear transformations of each other. Further $\psi\left(\mathbf{R}_{n}\right)$ and $\phi\left(\mathbf{S}_{n}\right)$ have the same limiting distribution under the null hypothesis and under any alternative (2.8) if

$$
\phi(t, u)=\psi(t, u)-\frac{1}{t} \int_{0}^{t} \psi(\tau, u) d \tau
$$


or

$$
\psi(t, u)=\phi(t, u)-\int_{t}^{1} \frac{1}{\tau} \phi(\tau, u) d \tau
$$

For the linear rank statistics,

$$
\sum_{i=1}^{n} a\left(\frac{i}{n}, \frac{R_{i n}}{n+1}\right) \quad \text { and } \quad \sum_{i=1}^{n}\left(a\left(\frac{i}{n}, \frac{S_{\text {in }}}{i+1}\right)-\frac{n}{i} \int_{0}^{\frac{i}{n}} a\left(\tau, \frac{S_{\text {in }}}{i+1}\right) d \tau\right)
$$

are asymptotically optimal test statistics agianst alternative (2.8).

Proof: Refer to Khmaladze \& Parjanadze (1986) and Parjanadze \& KHMALADZE (1986).

ExAmPLE: Returning to the Wilcoxon rank statistic, we consider the sequential rank version of this statistic. From (2.9) it follows that

$$
-\sum_{i=n_{1}+1}^{n} \frac{n_{1}}{i} \frac{S_{i}}{i+1}
$$

is asymptotically equivalent. We also note that if the size of the first sample is fixed and we keep adding new observations to the second sample, then for the sequential rank statistic we would only need to add the new summands to the existing statistic. 


\section{BIBLIOGRAPHY}

Billingsley, P. (1986), Probability and Measure, Probability and statistics, 2nd edn, Wiley. Cited on page 67.

Billingsley, P. (1999), Convergence of Probability Measures, Probability and statistics, 2nd edn, Wiley. Cited on pages 67, 73, 78, and 81.

Cantelli, F. P. (1933), 'Sulla determinazione empirica della legge di probabilita.', Giorn. Ist. Ital. Attuari 4, 421-424. Cited on page 2.

Chung, K., Erdos, P. \& Sirao, T. (1959), 'On the Lipschitz's condition for Brownian motion', Journal of the Mathematical Society of Japan 11(4). Cited on page 30 .

Chung, K.-L. (1949), 'An estimate concerning the Kolmogroff limit distribution', Transactions of the American Mathematical Society 67(1), 36-50. Cited on page 28.

Doob, J. (1949), 'Heuristic approach to the Kolmogorov-Smirnov theorems', Annals of Mathematical Statistics 20(3), 394-403. Cited on page 56.

Einmahl, J. (1993), Empirical and related processes with applications, Technical report, Eindhoven University of Technology. Cited on pages 13, 20, and 79 .

Feller, W. (1971a), An Introduction to Probability Theory and its Applications, Vol. 2, Wiley. Cited on pages 21 and 49.

Feller, W. (1971b), An Introduction to Probability Theory and its Applications, Vol. 1, Wiley. Cited on page 29.

Glivenko, V. (1933), 'Sulla determinazione empirica della legge di probabilita.', Giorn. Ist. Ital. Attuari 4, 92-99. Cited on page 2. 
Hajek, J., Sidak, Z. \& Sen, P. (1999), Theory of Rank Tests, Probability and mathematical statistics, 2nd edn, Academic press. Cited on page 122.

Hoeffding, W. (1963), 'Probability inequalities for sums of bounded random variables', Journal of the American Statistical Association 58(301), 13-30. Cited on page 4.

Hognas, G. (1986), 'Comparison of some non-linear autoregressive processes', Journal of Time Series Analysis 7(3), 205 - 211. Cited on page 99.

Ito, K. \& McKean Jr, H. P. (1974), Diffusion Processes and their Sample Paths, Springer-Verlag. Cited on page 22.

Khmaladze, E. \& Koul, H. (2004), 'Martingale transforms goodness-of-fit tests in regression models', The annals of statistics 32(3), 995-1034. Cited on page 103.

Khmaladze, E. \& Parjanadze, A. (1986), 'Functional limit theorems for linear statistics of sequential ranks', Notes Sci. Seminars POMI 204, 115-125. Cited on page 123.

Khmaladze, E. V. (2010), Sequential ranks, in 'Encyclopedia of statistics'. To appear in 2010. Cited on page 119.

Kosorok, M. R. (2008), Introduction to Empirical Processes and Semiparametric Inference, Statistics and Statistical Theory and Methods, Springer New York. Cited on page 4.

O’Brien, G. (1972), 'A note on comparisons of Markov processes.', Ann. Math. Statist. 43, 365-368. Cited on page 99.

Parjanadze, A. \& Khmaladze, E. (1986), 'On the asymptotic theory of statistics based on sequential ranks', Theory Probability Applications 31, 669682. Cited on page 123 .

Pflug, G. (1991), 'A note on the comparison of stationary laws of Markov processes.', Statist. Probab. Lett. (4), 331-334. Cited on page 99. 
Shorack, G. R. \& Wellner, J. A. (1986), Empirical Processes with Applications to Statistics, Wiley. Cited on pages 29, 33, 34, and 49.

Smirnov, N. N. (1944), 'An approximation to distribution laws of random quantities determined by empirical data.', Uspekhi Mat. Nauk. 10, 179-206. Cited on page 28.

van der Vaart, A. \& Wellner, J. (1996), Weak Convergence and Empirical Processes, Springer. Cited on pages 10, 12, 81, 82, and 83. 